\title{
Guidelines for pre-operative cardiac risk assessment and perioperative cardiac management in non-cardiac surgery
}

\author{
The Task Force for Preoperative Cardiac Risk Assessment and \\ Perioperative Cardiac Management in Non-cardiac Surgery of \\ the European Society of Cardiology (ESC) and endorsed by the \\ European Society of Anaesthesiology (ESA)
}

Authors/Task Force Members: Don Poldermans (Chairperson) (The Netherlands), Jeroen J. Bax (The Netherlands), Eric Boersma (The Netherlands), Stefan De Hert (The Netherlands),

Erik Eeckhout (Switzerland), Gerry Fowkes (UK), Bulent Gorenek (Turkey), Michael G. Hennerici (Germany), Bernard lung (France), Malte Kelm (Germany), Keld Per Kjeldsen (Denmark), Steen Dalby Kristensen (Denmark), Jose Lopez-Sendon (Spain), Paolo Pelosi (Italy), François Philippe (France), Luc Pierard (Belgium), Piotr Ponikowski (Poland), Jean-Paul Schmid (Switzerland), Olav F.M. Sellevold (Norway), Rosa Sicari (Italy), Greet Van den Berghe (Belgium) and Frank Vermassen (Belgium)

Additional Contributors: Sanne E. Hoeks (The Netherlands); Ilse Vanhorebeek (Belgium)

ESC Committee for Practice Guidelines (CPG): Alec Vahanian (Chairperson) (France), Angelo Auricchio (Switzerland), Jeroen J. Bax (The Netherlands), Claudio Ceconi (Italy), Veronica Dean (France), Gerasimos Filippatos (Greece), Christian Funck-Brentano (France), Richard Hobbs (UK), Peter Kearney (Ireland), Theresa McDonagh (UK), Keith McGregor (France), Bogdan A. Popescu (Romania), Zeljko Reiner (Croatia), Udo Sechtem (Germany), Per Anton Sirnes (Norway), Michal Tendera (Poland), Panos Vardas (Greece), Petr Widimsky (Czech Republic)

Document Reviewers: Raffaele De Caterina (CPG Review Coordinator) (Italy), Stefan Agewall (Norway), Nawwar Al Attar (France), Felicita Andreotti (Italy), Stefan D. Anker (Germany), Gonzalo Baron-Esquivias (Spain), Guy Berkenboom (Belgium), Laurent Chapoutot (France), Renata Cifkova (Czech Republic), Pompilio Faggiano (Italy), Simon Gibbs (UK), Henrik Steen Hansen (Denmark), Laurence Iserin (France), Carsten W. Israel (Germany), Ran Kornowski (Israel), Nekane Murga Eizagaechevarria (Spain), Mauro Pepi (Italy), Massimo Piepoli (Italy), Hans Joachim Priebe (Germany), Martin Scherer (Germany), Janina Stepinska (Poland), David Taggart (UK), Marco Tubaro (Italy)

European Journal of Anaesthesiology 2010, 27:92-137

Republished with permission from Eur Heart J. 2009; 22:2769-2812

The disclosure forms of all the authors and reviewers are available on the ESC website www.escardio.org/guidelines

Keywords: anaesthesiology, neurological disease, non-cardiac surgery, perioperative cardiac management, post-operative cardiac surveillance, pre-operative cardiac risk assessment, pre-operative cardiac testing, pre-operative coronary artery revascularization, pulmonary disease, renal disease

Correspondence to Don Poldermans, Department of Surgery, Erasmus Medical Center, Gravendijkwal 230, 3015 CE Rotterdam, The Netherlands Tel: +31 10703 4613; fax: +31 10436 4557; e-mail: d.poldermans@erasmusmc.nl

The content of these European Society of Cardiology (ESC) Guidelines has been published for personal and educational use only. No commercial use is authorized. No part of the ESC Guidelines may be translated or reproduced in any form without written permission from the ESC. Permission can be obtained upon submission of a written request to Oxford University Press, the publisher of the European Heart Journal and the party authorized to handle such permissions on behalf of the ESC.

Disclaimer: The ESC Guidelines represent the views of the ESC and were arrived at after careful consideration of the available evidence at the time they were written. Health professionals are encouraged to take them fully into account when exercising their clinical judgement. The guidelines do not, however, over-ride the individual responsibility of health professionals to make appropriate decisions in the circumstances of the individual patients, in consultation with that patient, and where appropriate and necessary the patient's guardian or carer. It is also the health professional's responsibility to verify the rules and regulations applicable to drugs and devices at the time of prescription. 


\section{Table of Contents}

List of acronyms and abbreviations . . . . . . . . . 93

Preamble . . . . . . . . . . . . . . . . 94

Introduction . . . . . . . . . . . . . . . . 95

Magnitude of the problem . . . . . . . . . 95

Impact of the ageing population . . . . . . . . . 96

Purpose. . . . . . . . . . . . . . . . . . 96

Pre-operative evaluation . . . . . . . . . . . . . . 97

Surgical risk for cardiac events . . . . . . . . . . . . 97

Functional capacity . . . . . . . . . . . . . . . 98

Risk indices. . . . . . . . . . . . . . . . . . . . 98

Biomarkers . . . . . . . . . . . . . . 100

Non-invasive testing . . . . . . . . . . . . . . . 101

Angiography . . . . . . . . . . . . . . . . 104

Risk reduction strategies. . . . . . . . . . . . . . 104

Pharmacological. . . . . . . . . . . . . . . 104

Revascularization . . . . . . . . . . . . 113

Specific diseases . . . . . . . . . . . . . . . . . 115

Chronic heart failure . . . . . . . . . . . . 116

Arterial hypertension. . . . . . . . . . . . . . . 117

Valvular heart disease . . . . . . . . . . . . 117

Arrhythmias . . . . . . . . . . . . . . . 118

Renal disease . . . . . . . . . . . . . . . . . . 119

Cerebrovascular disease. . . . . . . . . . 120

Pulmonary disease . . . . . . . . . . . . . . . 122

Perioperative monitoring . . . . . . . . . . . . . . 123

Electrocardiography . . . . . . . . . . . . . 123

Transoesophageal echocardiography . . . . . . . 124

Right heart catherization . . . . . . . . . . . . . 125

Disturbed glucose metabolism . . . . . . . . . . . 125

Anaesthesia . . . . . . . . . . . . . . . 127

Intraoperative anaesthetic management. . . . . . 127

Neuraxial techniques. . . . . . . . . . . . . . . . . 127

Post-operative pain management. . . . . . . . . 128

Putting the puzzle together. . . . . . . . . . . . . 128

References . . . . . . . . . . . . . . 131

List of acronyms and abbreviations

AAA

$\mathrm{ACC}$

ACE

ACS
AF

$\mathrm{AHA}$

AR

ARB

AS

BBSA

BNP

CABG

CARP

CASS

Cl

COPD

COX-2

CPET

CPG

CRP

CT

cTnl

cTnT

CVD

DECREASE

DES

DIPOM

DSE

ECG

ESC

$\mathrm{FEV}_{1}$

FRISC

HR

ICU

IHD

INR

LMWH

LQTS

LR

LV

MaVS

MET

MI atrial fibrillation

American Heart Association

aortic regurgitation

angiotensin receptor blocker

aortic stenosis

$\beta$-blocker in spinal anaesthesia

brain natriuretic peptide

coronary artery bypass grafting

Coronary Artery

Revascularization Prophylaxis

Coronary Artery Surgery Study

confidence interval

chronic obstructive pulmonary disease

cyclooxygenase-2

cardiopulmonary exercise testing

Committee for Practice Guidelines

C-reactive protein

computed tomography

cardiac troponin I

cardiac troponin $\mathrm{T}$

cardiovascular disease

Dutch Echocardiographic Cardiac Risk Evaluating Applying Stress Echo

drug-eluting stent

Diabetes Postoperative Mortality and Morbidity

dobutamine stress echocardiography

electrocardiography

European Society of Cardiology

forced expiratory volume in $1 \mathrm{~s}$

fast revascularization in instability in coronary disease

hazard ratio

intensive care unit

ischaemic heart disease

international normalized ratio

low molecular weight heparin

long QT syndrome

likelihood ratio

left ventricular

metoprolol after surgery

metabolic equivalent

myocardial infarction 


$\begin{array}{ll}\text { MR } & \text { mitral regurgitation } \\ \text { MRI } & \text { magnetic resonance imaging } \\ \text { MS } & \text { mitral stenosis } \\ \text { NICE-SUGAR } & \begin{array}{l}\text { Normoglycaemia in Intensive Care Evalu- } \\ \text { ation and Survival Using Glucose Algorithm } \\ \text { Regulation }\end{array}\end{array}$

NSTEMI non-ST-segment elevation myocardial infarction

NT-proBNP N-terminal pro-brain natriuretic peptide

NYHA

OPUS orbofiban in patients with unstable coronary syndromes

OR odds ratio

$\mathrm{PaCO}_{2} \quad$ mixed expired volume of alveolar and dead space gas

$\mathrm{PAH} \quad$ pulmonary arterial hypertension

$\mathrm{PCl} \quad$ percutaneous coronary intervention

PDA personal digital assistant

$\mathrm{PETCO}_{2}$ end-tidal expiratory $\mathrm{CO}_{2}$ pressure

POISE PeriOperative ISchaemic Evaluation trial

QUO-VADIS QUinapril On Vascular ACE and Determinants of ISchemia

ROC receiver operating characteristic

SD

SMVT

SPECT

SPVT

STEMI

SVT

SYNTAX

TACTICS

TIA

TIMI

TOE

UFH

$\mathrm{VCO}_{2}$

VE

VHD

VKA

$\mathrm{VO}_{2}$
VPB

VT

ventricular premature beat

ventricular tachycardia

\section{Preamble}

Guidelines and Expert Consensus Documents aim to present management and recommendations based on the relevant evidence on a particular subject in order to help physicians to select the best possible management strategies for the individual patient with a specific condition, taking into account not only the impact on outcome but also the risk-benefit ratio of particular diagnostic or therapeutic means. Guidelines are no substitutes for textbooks. The legal implications of medical guidelines have been discussed previously. ${ }^{1}$

A great number of Guidelines and Expert Consensus Documents have been issued in recent years by the European Society of Cardiology (ESC) and also by other organizations or related societies. Because of the impact on clinical practice, quality criteria for development of guidelines have been established in order to make all decisions transparent to the user. The recommendations for formulating and issuing ESG guidelines and Expert Consensus Documents can be found on the ESC website in the guidelines section (www.escardio.org).

In brief, experts in the field are selected and undertake a comprehensive review of the published evidence for management and/or prevention of a given condition. A critical evaluation of diagnostic and therapeutic procedures is performed, including assessment of the riskbenefit ratio. Estimates of expected health outcomes for larger societies are included, where data exist. The level of evidence and the strength of recommendation of particular treatment options are weighted and graded according to predefined scales, as outlined in Tables 1 and 2 .

The experts of the writing panels have provided disclosure statements of all relationships they may have which might be perceived as real or potential sources of conflicts of interest. These disclosure forms are kept on file at the

\section{Table 1 Classes of recommendations}

\begin{tabular}{|c|c|}
\hline $\begin{array}{l}\text { Classes of } \\
\text { recommendations }\end{array}$ & Definition \\
\hline Class I & $\begin{array}{l}\text { Evidence and/or general agreement that a given treatment } \\
\text { or procedure is beneficial, useful, and effective. }\end{array}$ \\
\hline Class II & $\begin{array}{l}\text { Conflicting evidence and/or a divergence of opinion about } \\
\text { the usefulness/efficacy of the given treatment or } \\
\text { procedure. }\end{array}$ \\
\hline Class Ila & $\begin{array}{l}\text { Weight of evidence/opinion is in favour of usefulness/ } \\
\text { efficacy. }\end{array}$ \\
\hline Class Ilb & $\begin{array}{l}\text { Usefulness/efficacy is less well established by evidence/ } \\
\text { opinion. }\end{array}$ \\
\hline Class III & $\begin{array}{l}\text { Evidence or general agreement that the given treatment } \\
\text { or procedure is not useful/effective, and in some } \\
\text { cases may be harmful. }\end{array}$ \\
\hline
\end{tabular}




\section{Table 2 Level of evidence}

\begin{tabular}{ll}
\hline Level of evidence A & $\begin{array}{c}\text { Data derived from multiple randomized clinical trials } \\
\text { or meta-analyses. } \\
\text { Data derived from a single randomized clinical trial } \\
\text { or large non-randomized studies. }\end{array}$ \\
Level of evidence C & $\begin{array}{c}\text { Consensus of opinion of the experts and/or small } \\
\text { studies, retrospective studies, registries. }\end{array}$
\end{tabular}

European Heart House, headquarters of the ESC. Any changes in conflict of interest that arise during the writing period must be notified to the ESC. The Task Force report is entirely supported financially by the ESC without any involvement of industry.

The ESC Committee for Practice Guidelines (CPG) supervises and coordinates the preparation of new Guidelines and Expert Consensus Documents produced by Task Forces, expert groups, or consensus panels. The Committee is also responsible for the endorsement process of these Guidelines and Expert Consensus Documents or statements. Once the document has been finalized and approved by all the experts involved in the Task Force, it is submitted to outside specialists for review. The document is revised, and finally approved by the CPG and subsequently published.

After publication, dissemination of the message is of paramount importance. Pocketsize versions and personal digital assistant (PDA)-downloadable versions are useful at the point of care. Some surveys have shown that the intended end-users are sometimes not aware of the existence of guidelines, or simply do not translate them into practice, so this is why implementation programmes for new guidelines form an important component of the dissemination of knowledge. Meetings are organized by the ESC, and are directed towards its member National Societies and key opinion leaders in Europe. Implementation meetings can also be undertaken at national levels, once the guidelines have been endorsed by the ESC member societies, and translated into the national language. Implementation programmes are needed because it has been shown that the outcome of disease may be favourably influenced by the thorough application of clinical recommendations. ${ }^{2}$

Thus, the task of writing Guidelines or Expert Consensus Documents covers not only the integration of the most recent research, but also the creation of educational tools and implementation programmes for the recommendations. The development of clinical guidelines and implementation into clinical practice can then only be completed if surveys and registries are performed to verify its use in real-life daily practices. Such surveys and registries also make it possible to evaluate the impact of implementation of the guidelines on patient outcomes. Guidelines and recommendations should help physicians and other healthcare providers to make decisions in their daily practice. However, the physician in charge of his/ her care must make the ultimate judgement regarding the care of an individual patient.

\section{Introduction \\ Magnitude of the problem}

The present guidelines focus on the cardiological management of patients undergoing non-cardiac surgery, i.e. patients in whom heart disease is a potential source of complications during surgery. The risk of perioperative complications depends on the condition of the patient prior to surgery, the prevalence of co-morbidities, and the magnitude and duration of the surgical procedure. ${ }^{3}$ More specifically, cardiac complications can arise in patients with documented or asymptomatic ischaemic heart disease (IHD), left ventricular (LV) dysfunction, and valvular heart disease (VHD) who undergo procedures that are associated with prolonged haemodynamic and cardiac stress. In the case of perioperative myocardial ischaemia, two mechanisms are important: (i) chronic mismatch in the supply-to-demand ratio of blood flow response to metabolic demand, which clinically resembles stable IHD due to a flow limiting stenosis in coronary conduit arteries; and (ii) coronary plaque rupture due to vascular inflammatory processes presenting as acute coronary syndromes (ACSs). Hence, although LV dysfunction may occur for various reasons in younger age groups, perioperative cardiac mortality and morbidity are predominantly an issue in the adult population undergoing major non-cardiac surgery.

The magnitude of the problem in Europe can best be understood in terms of (i) the size of the adult non-cardiac surgical cohort; and (ii) the average risk of cardiac complications within this cohort. Unfortunately, at a European level, no systematic data are available on the annual number and type of operations, nor on patient outcome. Information is collected at the national level in several countries, but data definitions, amount of data, and data quality vary greatly. In The Netherlands, with a population of 16 million, throughout 1991-2005, 250000 major surgical procedures were conducted on average annually in patients above the age of 20 years, implying an annual rate of $1.5 \%{ }^{4}$ When applied to Europe, with an overall population of 490 million, this figure translates into a crude estimate of 7 million major procedures annually in patients who present with cardiac risk.

Data on cardiac outcome can be derived from the few large-scale clinical trials and registries that have been undertaken in patients undergoing non-cardiac surgery. Lee et al. ${ }^{5}$ studied 4315 patients undergoing elective major non-cardiac procedures in a tertiary care teaching hospital throughout 1989-1994. They observed that 92 (2.1\%) patients suffered major cardiac complications, including cardiac death and myocardial infarction (MI). In a cohort of 108593 consecutive patients who under- 
went surgery throughout 1991-2000 in a university hospital in The Netherlands, perioperative mortality occurred in $1877(1.7 \%)$ patients, with a cardiovascular cause being identified in 543 cases $(0.5 \%){ }^{6}$ The Dutch Echocardiographic Cardiac Risk Evaluating Applying Stress Echo (DECREASE) -I, -II and -IV trials enrolled 3893 surgical patients throughout 1996-2008, and these comprised intermediate-risk and high-risk patients of whom 136 (3.5\%) suffered perioperative cardiac death or MI. ${ }^{7-9}$ A final piece of evidence with respect to patient outcome is derived from the Perioperative Ischaemic Evaluation (POISE) trial, which was conducted throughout 2002-2007, and enrolled 8351 patients undergoing non-cardiac surgery. ${ }^{10}$ Perioperative mortality occurred in 226 patients $(2.7 \%)$, of whom $133(1.6 \%)$ suffered cardiovascular death, whereas non-fatal MI was observed in another 367 (4.4\%) subjects. Differences in incidences between the studies are mainly explained by patient selection and endpoint MI definitions-major noncardiac surgery is associated with an incidence of cardiac death of between 0.5 and $1.5 \%$, and of major cardiac complications of between 2.0 and $3.5 \%$. When applied to the population in the European Union member states these figures translate into 150 000-250 000 life-threatening cardiac complications due to non-cardiac surgical procedures annually.

\section{Impact of the ageing population}

Within the next 20 years, the acceleration in ageing of the population will have a major impact on perioperative patient management. It is estimated that elderly people require surgery four times more often than the rest of the population. ${ }^{11}$ Although exact data regarding the number of patients undergoing surgery in Europe are lacking, it is estimated that this number will increase by $25 \%$ by 2020 , and for the same time period the elderly population will increase by $>50 \%$. The total number of surgical procedures will increase even faster because of the rising frequency of interventions with age. ${ }^{12}$ Results of the US National Hospital Discharge Survey show that, in general, the number of surgical procedures will increase in almost all age groups, but that the largest increase will occur in the middle aged and elderly (Table 3 ).

Table 3 Change in numbers of discharges for surgical procedures by age for the time periods $1994 / 95$ and 2004/05 as reported from the 2005 US National Hospital Discharge Survey (non-federal short-stay hospitals) ${ }^{15}$

\begin{tabular}{lrrr}
\hline & \multicolumn{2}{c}{$\begin{array}{c}\text { Number of procedures } \\
\text { (in thousands) }\end{array}$} & \\
\cline { 2 - 3 } Age (years) & $1994 / 95$ & $2004 / 05$ & \% change \\
\hline $18-44$ & 7311 & 7326 & +2.1 \\
$45-64$ & 4111 & 5210 & +26.7 \\
$65-74$ & 3069 & 3036 & -1.1 \\
75 and over & 3479 & 4317 & +24.1 \\
18 and over & 17969 & 19889 & +10.7 \\
\hline
\end{tabular}

Demographics of patients undergoing surgery show a trend towards an increasing number of elderly patients and co-morbidities. ${ }^{13}$ Although mortality from cardiac disease is decreasing in the general population, the prevalence of IHD, heart failure, and cardiovascular risk factors, especially diabetes, is increasing. Among the significant co-morbidities in elderly patients presenting for general surgery, cardiovascular disease (CVD) is the most prevalent. It is estimated from primary care data that in the 75-84 year age group 19\% of men and $12 \%$ of women have some degree of CVD. ${ }^{14}$ Age per se, however, seems to be responsible for only a small increase in the risk of complications; greater risks are associated with urgency and significant cardiac, pulmonary, and renal disease. The number of affected individuals is likely to be higher in countries with high CVD mortality, particularly in Central and Eastern Europe. These conditions should, therefore, have a greater impact on the evaluation of patient risk than age alone.

\section{Purpose}

Currently there are no official ESC guidelines on preoperative risk assessment and perioperative cardiac management. The objective is to endorse a standardized and evidence-based approach to perioperative cardiac management. The guidelines recommend a practical, stepwise evaluation of the patient, which integrates clinical risk factors and test results with the estimated stress of the planned surgical procedure. This results in an individualized cardiac risk assessment, with the opportunity to initiate medical therapy, coronary interventions, and specific surgical and anaesthetic techniques in order to optimize the patient's perioperative condition. Compared with the non-surgical setting, data from randomized clinical trials, which are the ideal evidence base for the guidelines, are sparse. Therefore, when no trials are available on a specific cardiac management regimen in the surgical setting, data from the non-surgical setting are used, and similar recommendations made, but with different levels of evidence. Emphasis is placed on the restricted use of prophylactic coronary revascularization, as this is rarely indicated simply to ensure the patient survives surgery. Pre-operative evaluation requires an integrated multidisciplinary approach from anaesthesiologists, cardiologists, internists, pulmonologists, geriatricians, and surgeons. Anaesthesiologists, who are experts on the specific demands of the proposed surgical procedure, usually coordinate the process.

Guidelines have the potential to improve post-operative outcome. However, as shown in an observational study of 711 vascular surgery patients from The Netherlands, adherence to guidelines is poor. ${ }^{16-18}$ Although 185 of a total of 711 patients (26\%) fulfilled the American College of Cardiology (ACC)/American Heart Association (AHA) guideline criteria for pre-operative non-invasive cardiac 
testing, clinicians had performed testing in only 38 of those cases $(21 \%){ }^{16}$ The guideline-recommended medical therapy for the perioperative period, namely the combination of aspirin and statins in all patients and $\beta$-blockers in patients with ischaemic heart disease, was followed in only $41 \%$ of cases. ${ }^{18}$ Significantly, the use of evidence-based medication during the perioperative period was associated with a reduction in 3-year mortality after adjustment for clinical characteristics [hazard ratio (HR), 0.65; 95\% confidence interval (CI), 0.45-0.94]. These data highlight the existence of a clear opportunity for improving the quality of care in this high-risk group of patients.

In addition to promoting an improvement in immediate perioperative care, guidelines should provide long-term advice, as patients should live long enough to enjoy the benefits of surgery. Following the development and introduction of perioperative cardiac guidelines, their effect on outcome should be monitored. The objective evaluation of changes in outcome will be an essential part of future perioperative guideline developments.

\section{Pre-operative evaluation}

\section{Surgical risk for cardiac events}

Cardiac complications after non-cardiac surgery depend not only on specific risk factors but also on the type of surgery and the circumstances under which it takes place. ${ }^{19}$ Surgical factors that influence cardiac risk are related to the urgency, magnitude, type, and duration of the procedure, as well as the change in body core temperature, blood loss, and fluid shifts. ${ }^{12}$

Every operation elicits a stress response. This response is initiated by tissue injury and mediated by neuroendocrine factors, and may induce tachycardia and hypertension. Fluid shifts in the perioperative period add to the surgical stress. This stress increases myocardial oxygen demand. Surgery also causes alterations in the balance between prothrombotic and fibrinolytic factors, resulting in hypercoagulability and possible coronary thrombosis (elevation of fibrinogen and other coagulation factors, increased platelet activation and aggregation, and reduced fibrinolysis). The extent of such changes is proportionate to the extent and duration of the intervention. All these factors may cause myocardial ischaemia and heart failure. Certainly in patients at elevated risk, attention to these factors should be given and lead, if indicated, to adaptations in the surgical plan.

Although patient-specific factors are more important than surgery-specific factors in predicting the cardiac risk for non-cardiac surgical procedures, the type of surgery cannot be ignored when evaluating a particular patient undergoing an intervention. ${ }^{6,20}$ With regard to cardiac risk, surgical interventions can be divided into low-risk, intermediate-risk, and high-risk groups with estimated 30-day cardiac event rates (cardiac death and MI) of $<1,1-5$, and $>5 \%$, respectively (Table 4 ). Although only a rough estimation, this risk stratification provides a good indication of the need for cardiac evaluation, drug treatment, and assessment of risk for cardiac events.

The high-risk group consists of major vascular interventions. In the intermediate-risk category the risk also depends on the magnitude, duration, location, blood loss, and fluid shifts related to the specific procedure. In the low-risk category the cardiac risk is negligible unless strong patient-specific risk factors are present.

The need for, and value of, pre-operative cardiac evaluation will also depend on the urgency of surgery. In the case of emergency surgical procedures, such as those for ruptured abdominal aortic aneurysm (AAA), major trauma, or for perforated viscus, cardiac evaluation will not change the course and result of the intervention but may influence the management in the immediate postoperative period. In non-emergent but urgent untreated surgical conditions such as bypass for acute limb ischaemia or treatment of bowel obstruction, the morbidity and mortality of the untreated underlying condition will outweigh the potential cardiac risk related to the intervention. In these cases, cardiological evaluation may influence the perioperative measures taken to reduce the cardiac risk, but will not influence the decision to perform the intervention. In some cases, the cardiac risk can also influence the type of operation and guide the choice to less invasive interventions, such as peripheral arterial angioplasty instead of infra-inguinal bypass, or extraanatomic reconstruction instead of an aortic procedure, even when these may yield less favourable results in the long term. Lastly, in some situations, the cardiac evaluation, in as far as it can reliably predict perioperative cardiac complications and estimate late survival, should be taken into consideration even when deciding whether to perform an intervention or not. This is the case in certain prophylactic interventions such as the treatment of small AAAs or asymptomatic carotid stenosis where the

\begin{tabular}{|c|c|c|}
\hline Low risk $<1 \%$ & Intermediate risk $1-5 \%$ & High risk $>5 \%$ \\
\hline $\begin{array}{l}\text { Breast } \\
\text { Dental } \\
\text { Endocrine } \\
\text { Eye } \\
\text { Gynaecology } \\
\text { Reconstructive } \\
\text { Orthopaedic_-minor } \\
\quad \text { (knee surgery] } \\
\text { Urologic_-minor }\end{array}$ & $\begin{array}{l}\text { Abdominal } \\
\text { Carotid } \\
\text { Peripheral arterial } \\
\quad \text { angioplasty } \\
\text { Endovascular aneurysm } \\
\quad \text { repair } \\
\text { Head and neck surgery } \\
\text { Neurological/orthopaedic_- } \\
\quad \text { major (hip and spine surgery) } \\
\text { Pulmonary renal/liver transplant } \\
\text { Urologic_-major }\end{array}$ & $\begin{array}{l}\text { Aortic and major } \\
\text { vascular surgery } \\
\text { Peripheral vascular } \\
\text { surgery }\end{array}$ \\
\hline
\end{tabular}

${ }^{\text {a }}$ Risk of myocardial infarction and cardiac death within 30 days after surgery. 
life expectancy of the patient and the risk of the operation are important factors in evaluating the potential benefit of the surgical intervention.

Vascular interventions are of specific interest, not only because they carry the highest risk of cardiac complications, explained by the high probability that the atherosclerotic process also affects the coronary arteries, but also because of the many studies that have shown that this risk can be influenced by adequate perioperative measures in these patients. Open aortic and infra-inguinal procedures have both to be considered as high-risk procedures. ${ }^{6}$ Although a less extensive intervention, infra-inguinal revascularization entails a cardiac risk similar to or even higher than aortic procedures. This can be explained by the higher incidence of diabetes, renal dysfunction, IHD, and advanced age in this patient group. This also explains why the risk related to peripheral artery angioplasties, which are minimally invasive procedures, is not negligible. Several randomized trials, as well as community-based studies, have shown that the cardiac risk is substantially lower after endovascular aortic aneurysm repair than after open repair. ${ }^{21}$ This can be related to the lesser tissue damage and the avoidance of aortic cross-clamping and post-operative ileus. However, long-term survival does not seem to be influenced by the surgical technique that is used, but is determined by the underlying cardiac disease. ${ }^{22}$ Carotid endarterectomy is considered to be an intermediate-risk procedure. Nevertheless, elevated cardiac risk and late survival should be taken into account in the decision-making process and can influence the choice between endarterectomy or stenting.

Laparoscopic procedures have the advantage of causing less tissue trauma and intestinal paralysis than open procedures, resulting in less incisional pain and diminished post-operative fluid shifts related to bowel paralysis. ${ }^{23}$ On the other hand, the pneumoperitoneum used in these procedures results in elevated intra-abdominal pressure and a reduction in venous return. It will result in a decrease in cardiac output and an increase in systemic vascular resistance. Therefore, cardiac risk in patients with heart failure is not diminished in patients undergoing laparoscopy compared with open surgery, and both should be evaluated in the same way. This is especially true in patients undergoing interventions for morbid obesity. ${ }^{24,25}$

Recommendation/statement on surgical risk estimate

\begin{tabular}{lll}
\hline Recommendation/statement & Class $^{\mathrm{a}}$ & Level $^{\mathrm{b}}$ \\
\hline Laparoscopic procedures demonstrate a cardiac & $\mathrm{I}$ & $\mathrm{A}$ \\
stress similar to open procedures and it is & & \\
recommended that patients be screened prior & \\
to intervention accordingly & \\
\hline
\end{tabular}

${ }^{a}$ Class of recommendation. ${ }^{\mathrm{b}}$ Level of evidence.

\section{Functional capacity}

Determination of functional capacity is considered to be a pivotal step in pre-operative cardiac risk assessment. Functional capacity is measured in metabolic equivalents (METs). One MET equals the basal metabolic rate. Exercise testing provides an objective assessment of functional capacity. Without testing, functional capacity can be estimated by the ability to perform the activities of daily living. Given that 1 MET represents metabolic demand at rest, climbing two flights of stairs demands 4 METs, and strenuous sports such as swimming $>10$ METS (Fig. 1).

The inability to climb two flights of stairs or run a short distance $(<4$ METs) indicates poor functional capacity and is associated with an increased incidence of postoperative cardiac events. After thoracic surgery, a poor functional capacity has been associated with an increased mortality (relative risk 18.7, 95\% CI 5.9-59). However, in comparison with thoracic surgery, a poor functional status was not associated with an increased mortality after other non-cardiac surgery (relative risk 0.47 , 95\% CI 0.09 2.5). ${ }^{28}$ This may reflect the importance of pulmonary function, strongly related to functional capacity, as a major predictor of survival after thoracic surgery. These findings were confirmed in a study of 5939 patients scheduled for non-cardiac surgery in which the prognostic importance of pre-operative functional capacity was measured in METs. ${ }^{29}$ Using receiver operating characteristic (ROC) curve analysis, the association of functional capacity with post-operative cardiac events or death showed an area under the ROC curve of just 0.664, compared with 0.814 for age. Considering the relatively weak association between functional capacity and post-operative cardiac outcome, what importance should we attach to functional capacity assessment in the pre-operative evaluation of the risk of non-cardiac surgery? When functional capacity is high, the prognosis is excellent, even in the presence of stable IHD or risk factors. ${ }^{30}$ In this case, perioperative management will rarely be changed as a result of further cardiac testing and the planned surgical procedure can proceed. Using functional capacity evaluation prior to surgery, the ability to climb two flights of stairs or run for a short distance indicated a good functional capacity. On the other hand, when functional capacity is poor or unknown, the presence and number of risk factors in relation to the risk of surgery will determine pre-operative risk stratification and perioperative management.

\section{Risk indices}

Effective strategies aimed at reducing the risk of perioperative cardiac complications should involve cardiac evaluation using medical history prior to the surgical procedure, for two main reasons. First, patients with an anticipated low cardiac risk-after thorough evaluationcan be operated on safely without further delay. It is 


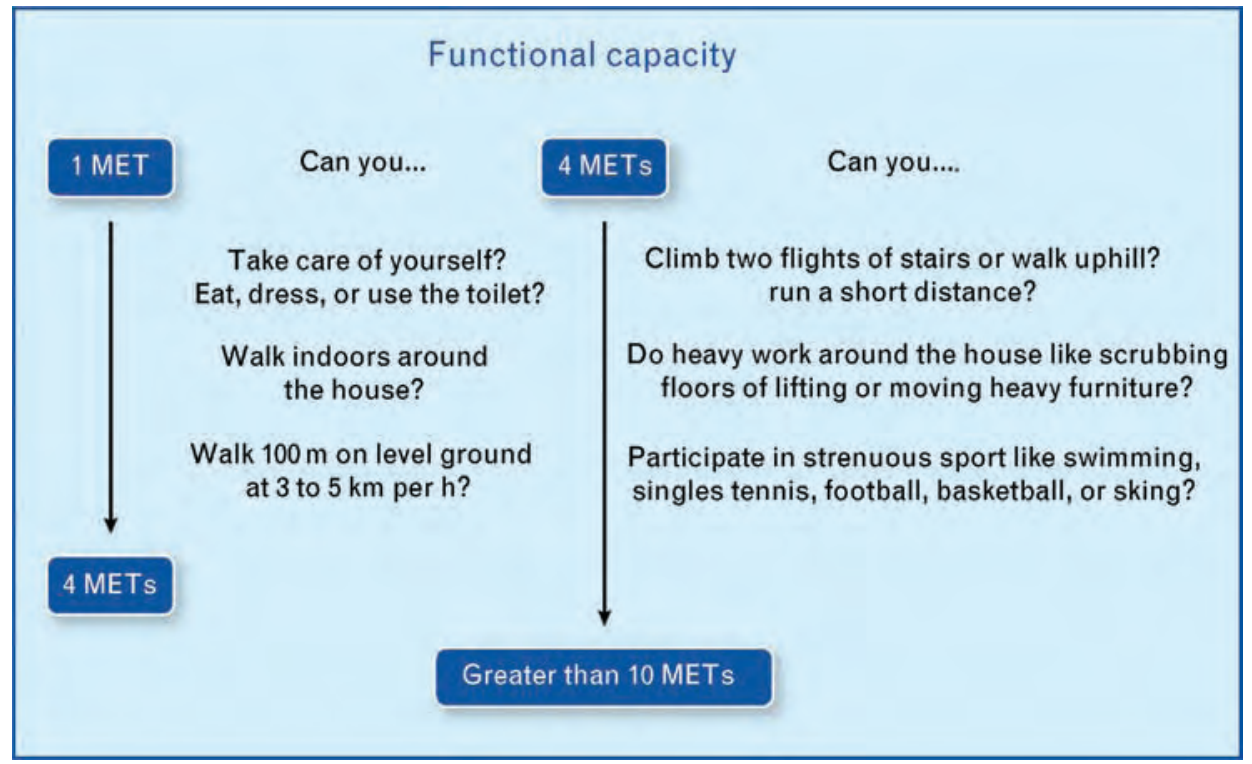

Estimated energy requirements for various activities. $\mathrm{km}$ per $\mathrm{h}$, kilometres per hour; MET, metabolic equivalent. Based on $\mathrm{Hlatky} \mathrm{et} \mathrm{al.}{ }^{26}$ and Fletcher et al. ${ }^{27}$

unlikely that risk reduction strategies can reduce the perioperative risk further. Secondly, risk reduction by pharmacological treatment is most cost-effective in patients with a suspected increased cardiac risk. Additional non-invasive cardiac imaging techniques are tools to identify patients at higher risk. However, imaging techniques should be reserved for those patients in whom test results would influence and change management. Obviously, the intensity of the pre-operative cardiac evaluation must be tailored to the patient's clinical condition and the urgency of the circumstances requiring surgery. When emergency surgery is needed, the evaluation must necessarily be limited. However, most clinical circumstances allow the application of a more extensive, systematic approach, with cardiac risk evaluation that is initially based on clinical characteristics and type of surgery, and then extended-if indicated-to resting electrocardiography (ECG), laboratory measurements, and non-invasive (stress) testing.

During the last 30 years, several risk indices have been developed, based on multivariable analyses of observational data, which represent the relationship between clinical characteristics and perioperative cardiac mortality and morbidity. The indices that were developed by Goldman (1977), Detsky (1986), and Lee (1999) became well known. ${ }^{5,31,32}$ The Lee index, which is in fact a modification of the original Goldman index, is considered by many clinicians and researchers to be the best currently available cardiac risk prediction index in non-cardiac surgery. It was developed using prospectively collected data on 2893 unselected patients (and validated in another 1422 patients) who underwent a wide spectrum of procedures. They were followed systematically throughout the post-operative phase for a range of clinically relevant cardiac outcomes. The Lee index contains five independent clinical determinants of major perioperative cardiac events: a history of IHD, a history of cerebrovascular disease, heart failure, insulin-dependent diabetes mellitus, and impaired renal function. High-risk type of surgery is the sixth factor that is included in the index. All factors contribute equally to the index (with 1 point each), and the incidence of major cardiac complications is estimated at $0.4,0.9,7$, and $11 \%$ in patients with an index of $0,1,2$, and $\geq 3$ points, respectively. The area under the ROC curve in the validation data set was 0.81 , indicating that the index has a high capability for discriminating between patients with and without a major cardiac event.

However, the patients studied by Lee $e t$ al. cannot be considered to be an average, unselected non-cardiac surgical cohort. Patients undergoing thoracic (12\%), vascular $(21 \%)$, and orthopaedic surgery $(35 \%)$ were overrepresented. Furthermore, despite its respectable size, the study was too underpowered to reveal a broad range of cardiac outcome determinants, as only 56 cardiac events were observed in the derivation cohort. Several external validation studies have suggested that the Lee index is probably suboptimal for identifying patients with multiple risk factors. ${ }^{6}$ In fact, the type of surgery was only classified as two subtypes: first, high-risk, including intraperitoneal, intrathoracic, and suprainguinal vascular 
Table 5 Lee index and Erasmus model: clinical risk factors used for pre-operative cardiac risk stratification ${ }^{5,6}$

\begin{tabular}{lcc}
\hline Clinical characteristics & Lee index & Erasmus model \\
\hline $\begin{array}{l}\text { IHD (angina pectoris and/or MI) } \\
\text { Surgical risk }\end{array}$ & $\begin{array}{c}\text { x } \\
\text { High-risk } \\
\text { surgery }\end{array}$ & $\begin{array}{l}\text { High, } \\
\text { intermediate-high, } \\
\text { intermediate-low, }\end{array}$ \\
& low risk \\
Heart failure & $\mathrm{x}$ & $\mathrm{x}$ \\
Stroke/transient ischaemic attack & $\mathrm{x}$ & $\mathrm{x}$ \\
Diabetes mellitus requiring insulin therapy & $\mathrm{x}$ & $\mathrm{x}$ \\
Renal dysfunction/haemodialysis & $\mathrm{x}$ & $\mathrm{x}$ \\
Age & & $\mathrm{x}$ \\
\hline
\end{tabular}

$\mathrm{IHD}$, ischaemic heart disease; Ml, myocardial infarction.

procedures; and, second, all remaining non-laparoscopic procedures, mainly including orthopaedic, abdominal, and other vascular procedures. Evidence exists that a more subtle classification, such as the Erasmus model, results in better risk discrimination. ${ }^{6}$ In this model, an extensive description of the type of surgery and age increased the prognostic value of the model for perioperative cardiac events (area under the ROC curve for the prediction of cardiovascular mortality increased from 0.63 to 0.85 ) (Table 5).

\begin{tabular}{|c|c|c|}
\hline Recommendations/statements & Class $^{a}$ & Level $^{b}$ \\
\hline $\begin{array}{l}\text { It is recommended that clinical risk indices be used for } \\
\text { post-operative risk stratification }\end{array}$ & 1 & $\mathrm{~B}$ \\
\hline $\begin{array}{l}\text { It is recommended that the Lee index model } \\
\text { applying six different variables for perioperative } \\
\text { cardiac risk be used }\end{array}$ & 1 & A \\
\hline
\end{tabular}

\section{Biomarkers}

A biological marker-biomarker-is a characteristic that can be objectively measured and evaluated and which is an indicator of abnormal biological and pathogenic processes or responses to therapeutic interventions. In the perioperative setting, biomarkers can be divided into markers focusing on myocardial ischaemia and damage, inflammation, and $\mathrm{LV}$ function.

Cardiac troponins $\mathrm{T}$ and $\mathrm{I}$ (cTnT and $\mathrm{c} T \mathrm{TnI}$ ) are the preferred markers for the diagnosis of MI because they demonstrate sensitivity and tissue specificity superior to other available biomarkers. ${ }^{33,34}$ The prognostic information is independent of, and complementary to, other important cardiac indicators of risk such as ST deviation and $\mathrm{LV}$ function. The prognostic significance of even small elevations in troponins has been independently confirmed in community-based studies and in clinical trials (TACTICS-TIMI 18, FRISC II, OPUS-TIMI), ${ }^{35,36}$ not only in high-risk, but also in intermediate-risk groups. cTnI and CTnT seem to be of similar value for risk assessment in acute coronary syndrome (ACS) in the presence and absence of renal failure. ${ }^{33}$ The prognosis for all-cause death in patients with end-stage renal disease and with even minor elevations in cTnT is 2-5 times worse than for those with undetectable values. Existing evidence suggests that even small increases in c'TnT in the perioperative period reflect clinically relevant myocardial injury with worsened cardiac prognosis and outcome. ${ }^{37}$ The development of new biomarkers, including high-sensitivity troponins, will further enhance the assessment of myocardial damage. It should be noted that troponin elevation may be observed in many other conditions. The diagnosis of non-STsegment elevation myocardial infarction (NSTEMI) should never be made solely on the basis of biomarkers.

Inflammatory markers might identify pre-operatively those patients with an increased risk of unstable coronary plaque. C-reactive protein (CRP) is an acute-phase reactant produced in the liver. CRP is also expressed in smooth muscle cells within diseased atherosclerotic arteries and has been implicated in many aspects of atherogenesis and plaque vulnerability, including expression of adhesion molecules, induction of nitric oxide, altered complement function, and inhibition of intrinsic fibrinolysis. ${ }^{38}$ However, in the surgical setting, no data are currently available using CRP as a marker for the initiation of risk reduction strategies.

Brain natriuretic peptide (BNP) and N-terminal pro-BNP (NT-proBNP) are produced in cardiac myocytes in response to increases in myocardial wall stress. This may occur at any stage of heart failure, independently of the presence or absence of myocardial ischaemia. Plasma BNP and NT-proBNP have emerged as important prognostic indicators in patients with heart failure, ACS, and stable IHD in non-surgical settings. ${ }^{39-41}$ Pre-operative BNP and NT-proBNP levels have additional prognostic value for long-term mortality and for cardiac events after major non-cardiac vascular surgery. ${ }^{42-46}$

Data on pre-operative biomarker use from prospective controlled trials are sparse. Based on the present data, routine assessment of serum biomarkers for patients undergoing non-cardiac surgery cannot be proposed for routine use as an index of cell damage.

\begin{tabular}{lll} 
Recommendations/statements on biomarkers & & \\
\hline Recommendations/statements & Class $^{\mathrm{a}}$ & Level $^{\mathrm{b}}$ \\
\hline $\begin{array}{l}\text { NT-proBNP and BNP measurements should be } \\
\text { considered for obtaining independent prognostic } \\
\text { information for perioperative and late cardiac events }\end{array}$ & Ila & B \\
in high-risk patients & & \\
$\begin{array}{l}\text { Routine biomarker sampling to prevent cardiac events } \\
\text { is not recommended }\end{array}$ & III & $\mathrm{C}$ \\
\hline
\end{tabular}

BNP, brain natriuretic peptide; NT-proBNP, N-terminal pro-brain natriuretic peptide. ${ }^{a}$ Class of recommendation. ${ }^{b}$ Level of evidence. 


\section{Non-invasive testing}

Pre-operative non-invasive testing aims at providing information on three cardiac risk markers: LV dysfunction, myocardial ischaemia, and heart valve abnormalities, all major determinants of adverse post-operative outcome. $\mathrm{LV}$ function is assessed at rest, and various imaging modalities are available. For myocardial ischaemia detection, exercise ECG and non-invasive imaging techniques may be used. The overall theme is that the diagnostic algorithm for risk stratification of myocardial ischaemia and $\mathrm{LV}$ function should be similar to that proposed for patients in the non-surgical setting with known or suspected IHD. ${ }^{47}$ Non-invasive testing should not only be considered for coronary artery revascularization but also for patient counselling, change of perioperative management in relation to type of surgery, anaesthetic technique, and long-term prognosis. Echocardiography is preferred for evaluation of valve disease (see section on specific diseases, subheading valvular heart disease).

\section{Non-invasive testing of cardiac disease}

\section{Electrocardiography}

The 12-lead ECG is commonly performed as part of preoperative cardiovascular risk assessment in patients undergoing non-cardiac surgery. In IHD patients, the pre-operative electrocardiogram contains important prognostic information and is predictive of long-term outcome independent of clinical findings and perioperative ischaemia. ${ }^{48}$ However, the electrocardiogram may be normal or non-specific in a patient with either ischaemia or infarction. The routine use of ECG prior to all types of surgery is a subject of increasing debate. A retrospective study investigated 23036 patients scheduled for 28457 surgical procedures; patients with abnormal ECG findings had a greater incidence of cardiovascular death than those with normal ECG results (1.8\% vs. $0.3 \%)$. In patients who underwent low-risk or low- to intermediate-risk surgery, the absolute difference in the incidence of cardiovascular death between those with and without ECG abnormalities was only $0.5 \%$. $^{49}$

\begin{tabular}{lll} 
Recommendations on ECG & & \\
\hline Recommendations & Class $^{\mathrm{a}}$ & Level $^{\mathrm{b}}$ \\
\hline $\begin{array}{l}\text { Pre-operative ECG is recommended for patients who have } \\
\text { risk factor(s) and are scheduled for intermediate- or } \\
\text { high-risk surgery }\end{array}$ & B & \\
$\begin{array}{l}\text { Pre-operative ECG should be considered for patients } \\
\text { who have risk factor(s) and are scheduled for low-risk } \\
\text { surgery }\end{array}$ & Illa & B \\
$\begin{array}{l}\text { Pre-operative ECG may be considered for patients } \\
\text { who have no risk factor and are scheduled for } \\
\text { intermediate-risk surgery }\end{array}$ & Blb \\
$\begin{array}{l}\text { Pre-operative ECG is not recommended for patients } \\
\text { who have no risk factor and are scheduled for } \\
\text { low-risk surgery }\end{array}$ & III & B \\
\hline
\end{tabular}

ECG, electrocardiography. ${ }^{a}$ Class of recommendation. ${ }^{b}$ Level of evidence.

\section{Assessment of left ventricular function}

Resting $\mathrm{LV}$ function can be evaluated before non-cardiac surgery by radionuclide ventriculography, gated single photon emission computed tomography (SPECT) imaging, echocardiography, magnetic resonance imaging (MRI), or multislice computed tomography (C'T), with similar accuracy. ${ }^{50}$ Routine echocardiography is not recommended for the pre-operative evaluation of $\mathrm{LV}$ function, but may be performed in asymptomatic patients undergoing high-risk surgery. A meta-analysis of the available data demonstrated that an $\mathrm{LV}$ ejection fraction of $<35 \%$ had a sensitivity of $50 \%$ and a specificity of $91 \%$ for prediction of perioperative non-fatal MI or cardiac death. ${ }^{51}$ The limited predictive value of $\mathrm{LV}$ function assessment for perioperative outcome may be related to the failure to detect severe underlying IHD. Recommendations for the pre-operative evaluation of (asymptomatic) patients with cardiac murmurs are discussed in the section on VHD.

Recommendations on resting echocardiography

\begin{tabular}{|c|c|c|}
\hline Recommendations & Class $^{a}$ & Level $^{b}$ \\
\hline $\begin{array}{l}\text { Rest echocardiography for LV assessment should be } \\
\text { considered in patients undergoing high-risk surgery }\end{array}$ & Ila & C \\
\hline $\begin{array}{l}\text { Rest echocardiography for LV assessment in } \\
\text { asymptomatic patients is not recommended }\end{array}$ & III & $B$ \\
\hline
\end{tabular}

LV, left ventricular. ${ }^{a}$ Class of recommendation. ${ }^{b}$ Level of evidence.

\section{Non-invasive testing of ischaemic heart disease}

Physiological exercise using a treadmill or bicycle ergometer is the preferred method for detection of ischaemia. Physiological exercise provides an estimate of functional capacity, provides blood pressure and heart rate response, and detects myocardial ischaemia through ST-segment changes. The accuracy of exercise ECG varies significantly among studies. Meta-analysis of the reported studies using treadmill testing in vascular surgery patients showed a rather low sensitivity $(74 \%, 95 \%$ CI $60-88 \%)$ and specificity $(69 \%$, 95\% CI 60-78\%), comparable with daily clinical practice. ${ }^{51}$ The positive predictive value was as low as $10 \%$, but the negative predictive value was very high (98\%). However, risk stratification with exercise is not suitable for patients with limited exercise capacity due to their inability to reach an ischaemic threshold. Furthermore, pre-existing ST-segment abnormalities, especially in the pre-cordial leads $V_{5}$ and $V_{6}$ at rest, hamper reliable ST-segment analysis. A gradient of severity in the test result relates to the perioperative outcome: the onset of a myocardial ischaemic response at low exercise workloads is associated with a significantly increased risk of perioperative and long-term cardiac events. In contrast, the onset of myocardial ischaemia at high workloads is associated with significantly less risk. ${ }^{30}$ Pharmacological stress testing with either nuclear perfusion imaging or echocardiography is more suitable in patients with limited physical capabilities. 
The role of myocardial perfusion imaging for pre-operative risk stratification is well established. In patients with limited exercise capacity, pharmacological stress (dipyridamole, adenosine, or dobutamine) is an alternative stressor. Images reflect myocardial blood distribution at the time of injection. Studies are performed both during stress and at rest to determine the presence of reversible defects, reflecting jeopardized ischaemic myocardium, or fixed defects, reflecting scar or non-viable tissue.

The prognostic value of the extent of ischaemic myocardium, using semi-quantitative dipyridamole myocardial perfusion imaging, has been investigated in a meta-analysis of studies in vascular surgery patients. ${ }^{52}$ Study endpoints were perioperative cardiac death and MI. The authors included nine studies, totalling 1179 vascular surgery patients, with a 7\% 30-day event rate. In this analysis, reversible ischaemia in $<20 \%$ of the $\mathrm{LV}$ myocardium did not change the likelihood of perioperative cardiac events, compared with those without ischaemia. Patients with more extensive reversible defects were at increased risk: $20-29 \%$ reversibility [likelihood ratio (LR) 1.6, 95\% CI 1.0-2.6], 30-39\% reversibility (LR 2.9, 95\% CI $1.6-5.1$ ), $40-49 \%$ reversibility (LR $2.9,95 \%$ CI $1.4-6.2$ ), and $\geq 50 \%$ reversibility (LR $11,95 \%$ CI $5.8-20$ ).

A second meta-analysis, which assessed the prognostic value of six diagnostic tests, reported a sensitivity of $83 \%$ (95\% CI 77-92\%) with a much lower specificity of $47 \%$ (95\% CI, 41-57\%) for myocardial perfusion imaging. ${ }^{51-53}$ The positive and negative predictive values were 11 and $97 \%$, respectively.

A third meta-analysis pooled the results of 10 studies evaluating dipyridamole thallium-201 imaging in vascular surgery candidates over a 9 -year period $(1985-1994) .{ }^{53}$ The 30-day cardiac death or non-fatal MI rates were $1 \%$ in patients with normal test results, $7 \%$ in patients with fixed defects, and $9 \%$ in patients with reversible defects on thallium-201 imaging. Moreover, three out of the 10 studies analysed used semi-quantitative scoring, demonstrating a higher incidence of cardiac events in patients with two or more reversible defects.

Overall, the positive predictive value of reversible defects for perioperative death or MI has decreased over recent years. This is probably related to changes in perioperative management and surgical procedures, resulting in a reduced cardiac event rate in patients with myocardial ischaemia as detected by pre-operative cardiac stress tests. However, because of the high sensitivity of nuclear imaging studies for detecting IHD, patients with a normal scan have an excellent prognosis. Myocardial perfusion imaging using dobutamine stress has a good safety profile. Hypotension, a systolic blood pressure decrease of $\geq 40 \mathrm{mmHg}$, occurred in $3.4 \%$, and serious cardiac arrhythmias in $3.8 \%$ of cases, in a consecutive series of 1076 patients. All arrhythmias terminated either spontaneously or after metoprolol administration. ${ }^{54}$

Stress echocardiography using exercise or pharmacological (dobutamine, dipyridamole) stress has been widely used for pre-operative cardiac risk evaluation. The test combines information on $\mathrm{LV}$ function at rest, heart valve abnormalities, and the presence and extent of stressinducible ischaemia. ${ }^{55}$ In one study, 530 patients were enrolled to evaluate the incremental value of dobutamine stress echocardiography (DSE) for the assessment of cardiac risk before non-vascular surgery. ${ }^{56}$ Multivariable predictors of post-operative events in patients with ischaemia were found to be a history of heart failure [odds ratio (OR) 4.7, 95\% CI 1.6-14.0] and ischaemic threshold $<60 \%$ of age-predicted maximal heart rate (OR 7.0, 95\% CI 2.8-17.6). DSE identified 60\% of patients as low risk (no ischaemia), $32 \%$ as intermediate risk (ischaemic threshold $\geq 60 \%$ ), and $8 \%$ as high risk (ischaemic threshold $<60 \%$ ); post-operative event rates were 0,9 , and $43 \%$, respectively. A recent meta-analysis showed that the sensitivity and specificity of DSE for perioperative cardiac death and MI are high (85 and 70\%, respectively). ${ }^{51}$ DSE can be performed safely with reasonable patient tolerance [incidence of cardiac arrhythmias and hypotension (defined as a systolic blood pressure decrease of $\geq 40 \mathrm{mmHg}$ )]. DSE has some limitations, e.g. it should not be used in patients with severe arrhythmias, significant hypertension, large thrombus-laden aortic aneurysms, or hypotension.

In general, stress echocardiography has a high negative predictive value (between 90 and 100\%): a negative test is associated with a very low incidence of cardiac events and indicates a safe surgical procedure. However, the positive predictive value is relatively low (between 25 and $45 \%$ ); this means that the post-surgical probability of a cardiac event is low, despite wall motion abnormality detection during stress echocardiography.

In a meta-analysis of 15 studies comparing dipyridamole thallium-201 imaging and DSE for risk stratification before vascular surgery, it was demonstrated that the prognostic value of stress imaging abnormalities for perioperative ischaemic events is comparable when using available techniques, but that the accuracy varies with IHD prevalence. ${ }^{53}$ In patients with a low incidence of IHD, the diagnostic accuracy is reduced compared with those with a high incidence of IHD.

MRI can also be used for detection of ischaemia; both perfusion and wall motion can be detected during stress and at rest. ${ }^{57}$ Ischaemia, more than IHD, is associated with adverse post-operative cardiac events. Therefore, functional testing is preferred to the detection of anatomical stenosis. The accuracy for assessment of ischaemia is high, with a sensitivity of $83 \%$ (95\% CI 79-88\%) 
and specificity of $86 \%$ (95\% CI $81-91 \%$ ) when wall motion is used (14 studies, 754 patients). When perfusion is added on top of wall motion abnormalities (24 studies, 1516 patients), sensitivity in the assessment of ischaemia increases to $91 \%$ (95\% CI 88-94\%); however, specificity decreases to $81 \%$ (95\% CI 77-85\%). MRI with dobutamine stress was used in 102 patients undergoing major non-cardiac surgery. ${ }^{58} \mathrm{New}$ wall motion abnormalities were used as a marker of ischaemia. Applying multivariable analysis, myocardial ischaemia was the strongest predictor of perioperative cardiac events (death, MI, and heart failure). MRI enabled non-invasive angiography and meta-analysis of existing data to be undertaken, using IHD detected by coronary angiography as a reference, and demonstrated sensitivity and specificity of $75 \%$ (95\% CI 68-80\%) and 85\% (95\% CI 78-90\%), respectively, on a vessel basis (16 studies, 2041 vessels); on a patient basis (13 studies, 607 subjects), sensitivity and specificity were $88 \%$ (95\% CI 82-92\%) and 56\% (95\% CI $53-68 \%)$ respectively. ${ }^{59}$ Currently no data are available in the setting of pre-operative risk stratification.

CT can be used to detect coronary calcium, which reflects coronary atherosclerosis. In addition, both electron beam and multi-slice CT have been used for non-invasive angiography, and a meta-analysis of existing data, using IHD detected by coronary angiography as a reference, demonstrated a sensitivity and a specificity of $82 \%(95 \%$ CI 80-85\%) and 91\% (95\% CI 90-92\%), respectively, on a vessel basis (eight studies, 2726 vessels); on a patient basis (21 studies, 1570 patients), sensitivity and specificity were $96 \%(95 \%$ CI $94-98 \%)$ and $74 \%$ (95\% CI 65$84 \%$ ), respectively. ${ }^{60}$ Data in the setting of pre-operative risk stratification are not yet available. A word of caution should be given with respect to the risk of radiation. ${ }^{61}$ In patients undergoing heart valve surgery, CT angiography has been used to exclude concomitant IHD, thereby avoiding the need for invasive coronary angiography. ${ }^{62}$ This approach may also be of use for pre-operative risk stratification; however, currently no data are available in the setting of pre-operative risk stratification.

How can these data be put into a practical algorithm? Testing should be performed only if it changes perioperative management. Patients with extensive stressinduced ischaemia represent a high-risk population in whom standard medical therapy appears to be insufficient to prevent perioperative cardiac events. ${ }^{63}$ Pre-operative testing may be considered in high-risk surgery patients with fewer than three clinical risk factors. However, in these patients, the beneficial effect of cardioprotective therapy appears to be sufficient to preclude pre-operative stress testing. The results of the randomized, multicentre DECREASE-II study showed that the perioperative cardiac event rate of vascular surgery patients on $\beta$ blocker therapy was already so reduced that test results and subsequent alteration in perioperative management were redundant. ${ }^{8}$ No differences in cardiac death and MI at 30 days were observed between 770 patients assigned to no cardiac stress testing vs. testing (1.8 vs. $2.3 \%$; OR 0.78 ; 95\% CI $0.28-2.1)$. Importantly, pre-operative testing delayed surgery for $>3$ weeks. Likewise, similar recommendations are given for intermediate-risk surgery patients, although no data from randomized trials are available. Considering the low event rate of patients scheduled for low-risk surgery, it is unlikely that test results in cardiac-stable patients will alter perioperative management.

\begin{tabular}{lll} 
Recommendations on stress testing prior to surgery & \\
\hline Recommendations & Class $^{\mathrm{a}}$ & Level $^{\mathrm{b}}$ \\
\hline $\begin{array}{l}\text { Stress testing is recommended in high-risk surgery } \\
\text { patients with } \geq 3 \text { clinical factors }\end{array}$ & $\mathrm{I}$ & $\mathrm{C}$ \\
$\begin{array}{l}\text { Stress testing may be considered in high-risk surgery } \\
\text { patients with } \leq 2 \text { clinical factors }\end{array}$ & $\mathrm{Ilb}$ & $\mathrm{B}$ \\
$\begin{array}{l}\text { Stress testing may be considered in intermediate-risk } \\
\text { surgery }\end{array}$ & $\mathrm{Ilb}$ & $\mathrm{C}$ \\
\begin{tabular}{l} 
Stress testing is not recommended in low-risk surgery \\
\hline
\end{tabular}
\end{tabular}

${ }^{\mathrm{a}}$ Class of recommendation. ${ }^{\mathrm{b}}$ Level of evidence. ${ }^{\mathrm{c}}$ Clinical risk factors are presented in Table 13.

\section{Integrated assessment of cardiopulmonary function}

Cardiopulmonary exercise testing (CPET) provides a global assessment of the integrated response to exercise involving the pulmonary, cardiovascular, and skeletal muscle systems. CPET is a programmed exercise test on either a cycle ergometer or a treadmill during which inspired and expired gases are measured through a facemask or a mouthpiece. This test provides information on oxygen uptake and utilization. ${ }^{64}$ The most commonly used data from this test are $\mathrm{O}_{2}$ consumption at peak exercise $\left(\mathrm{VO}_{2 \text { peak }}\right)$ and at anaerobic threshold $\left(\mathrm{VO}_{2 \mathrm{AT}}\right)$, defined as the point when metabolic demands exceed oxygen delivery, and anaerobic metabolism begins to occur. The thresholds for classifying patients as low risk are usually taken as $\mathrm{VO}_{2 \text { peak }}>15 \mathrm{~mL} / \mathrm{kg} / \mathrm{min}$ and $\mathrm{VO}_{2 \mathrm{AT}}$ $>11 \mathrm{~mL} / \mathrm{kg} / \mathrm{min}$. These thresholds roughly equate to 4 METs. ${ }^{65}$ CPET before lung resection may help in stratifying the surgical risk and optimizing perioperative care. In a cohort of 204 consecutive patients who had undergone pulmonary lobectomy or pneumonectomy, a $\mathrm{VO}_{2 \text { peak }}<20 \mathrm{~mL} / \mathrm{kg} / \mathrm{min}$ was a predictor of pulmonary complications, cardiac complications, and mortality; a $\mathrm{VO}_{2 \text { peak }}<12 \mathrm{~mL} / \mathrm{kg} / \mathrm{min}$ was associated with a 13 -fold higher rate of mortality. ${ }^{66}$ In a study of 187 elderly patients $\mathrm{VO}_{2 \mathrm{AT}}$ was measured before major abdominal surgery. ${ }^{67}$ The overall mortality was $5.9 \%$. Patients who had a $\mathrm{VO}_{2 \mathrm{AT}}<11 \mathrm{~mL} / \mathrm{kg} / \mathrm{min}(n=55)$ had a mortality of $18 \%$ compared with those who had a $\mathrm{VO}_{2 \mathrm{AT}}>11 \mathrm{~mL} / \mathrm{kg} /$ $\min (n=132)$ whose mortality was $0.8 \%$ (risk ratio 24 , 95\% CI 3.1-183). In patients who exhibited signs of myocardial ischaemia during testing, the mortality was $42 \%$ for patients whose $\mathrm{VO}_{2 \mathrm{AT}}$ was $<11 \mathrm{~mL} / \mathrm{kg} / \mathrm{min}$ and only $4 \%$ for those whose $\mathrm{VO}_{2 \mathrm{AT}}$ was $>11 \mathrm{~mL} / \mathrm{kg} / \mathrm{min}$ 
$(P<0.001)$. CPET also carries accurate prognostic information in the setting of heart failure patients: an abnormally high relationship between minute ventilation (VE) and carbon dioxide production $\left(\mathrm{VCO}_{2}\right)$, expressed as the $\mathrm{VE} / \mathrm{VCO}_{2}$ slope measured between the onset of loaded exercise and the end of the isocapnic buffering period, identified by the rise in the $\mathrm{VE} / \mathrm{VCO}_{2}$ slope and the reduction of end-tidal expiratory $\mathrm{CO}_{2}$ pressure $\left(\mathrm{PETCO}_{2}\right)$ (or mixed expired value of alveolar and dead space gas, $\mathrm{PaCO}_{2}$ ), is associated with a poor outcome, as is an oscillatory pattern of ventilation during exercise, defined as cyclic fluctuations in minute ventilation at rest that persist during effort. ${ }^{68}$ There are potential discrepancies between a CPET and functional assessment using METs that preclude a widespread use of CPET. Non-cardiac and non-respiratory factors such as skeletal muscle function and physical training can underestimate aerobic metabolic activity. A further consideration must be the availability of CPET testing, which at present is not available in all centres. The role of CPET in preoperative risk assessment has not been established and CPET should not be considered to be a substitute for stress testing in routine practice.

\section{Angiography}

Coronary angiography is a well-established invasive diagnostic procedure but is rarely indicated to assess the risk of non-cardiac surgery. There is a lack of information derived from randomized clinical trials on its usefulness in patients scheduled for non-cardiac surgery. Moreover, adopting an invasive coronary angiography assessment may cause an unnecessary and unpredictable delay in an already planned surgical intervention. Nevertheless, IHD may be present in a significant number of patients in whom non-cardiac surgery is indicated. In patients with known IHD, indications for pre-operative coronary angiography and revascularization are similar to angiography indications in the non-surgical setting. ${ }^{4769-71}$ The control of ischaemia before surgery, either medically or with intervention, is recommended whenever noncardiac surgery procedures can be delayed.

\section{Recommendations on pre-operative coronary angiography}

\begin{tabular}{lll}
\hline Recommendations & Class $^{\mathrm{a}}$ & Level $^{\mathrm{b}}$ \\
\hline $\begin{array}{l}\text { Pre-operative angiography is recommended in patients } \\
\text { with acute STEMI }\end{array}$ & $\mathrm{I}$ & $\mathrm{A}$ \\
$\begin{array}{c}\text { Pre-operative angiography is recommended in patients } \\
\text { with NSTEMI and unstable angina }\end{array}$ & $\mathrm{I}$ & $\mathrm{A}$ \\
$\begin{array}{c}\text { Pre-operative angiography is recommended in patients } \\
\text { with angina not controlled with adequate medical therapy }\end{array}$ & $\mathrm{I}$ & $\mathrm{A}$ \\
$\begin{array}{c}\text { Pre-operative angiography may be considered in cardiac- } \\
\text { stable patients undergoing high-risk surgery }\end{array}$ & $\mathrm{Ilb}$ & $\mathrm{B}$ \\
$\begin{array}{c}\text { Pre-operative angiography may be considered in cardiac- } \\
\text { stable patients undergoing intermediate-risk surgery }\end{array}$ & $\mathrm{Ilb}$ & $\mathrm{C}$ \\
$\begin{array}{c}\text { Pre-operative angiography is not recommended in } \\
\text { cardiac-stable patients undergoing low-risk surgery }\end{array}$ & $\mathrm{III}$ & $\mathrm{C}$
\end{tabular}

NSTEMI, non-ST-segment elevation myocardial infarction; STEMI, ST-segment elevation myocardial infarction. ${ }^{\text {a }}$ Class of recommendation. ${ }^{b}$ Level of evidence.

\section{Risk reduction strategies Pharmacological}

The occurrence of MI during the intra- or early postoperative period is frequently preceded by prolonged or recurrent myocardial ischaemia. The stress of surgery and anaesthesia may trigger ischaemia through an imbalance between myocardial oxygen demand and supply. Besides specific risk reduction strategies adapted to patient characteristics and the type of surgery, pre-operative evaluation is an opportunity to check and optimize the control of all cardiovascular risk factors.

\section{$\boldsymbol{\beta}$-Blockers}

During the perioperative period, there is a catecholamine surge, resulting in an increased heart rate and myocardial contractility and subsequent increased myocardial oxygen consumption. The main rationale for perioperative $\beta$ blocker use is to decrease myocardial oxygen consumption by reducing heart rate, resulting in a lengthening of the diastolic filling period, and decreased myocardial contractility. ${ }^{72}$ Additional cardioprotective factors are redistribution of coronary blood flow to the subendocardium, plaque stabilization, and fibrillation. ${ }^{72}$ and other drugs that lower the heart rate can reduce perioperative myocardial ischaemia as assessed by continuous ST-segment monitoring. ${ }^{73}$ However, whether this translates into a clinical benefit can be established only through trials analysing the incidence of cardiovascular events. Seven multicentre randomized trials evaluating the effect of perioperative $\beta$-blockade on clinical endpoints have been published in peer-reviewed journals (Table 6 and Fig. 2). ${ }^{9,10,74-78}$

Three trials targeted patients at high risk for perioperative complications because of the type of surgery, the presence of IHD, or risk factors for perioperative cardiac complications. ${ }^{9,76,78}$ Three other trials did not require the presence of clinical risk factors, except for diabetes in one case. ${ }^{74,75,77}$ The POISE trial included patients with a wide spectrum of risk of perioperative cardiac complications. ${ }^{10}$

The first trial randomized 200 patients with at least two risk factors for IHD or with known IHD, who were scheduled for non-cardiac surgery under general anaesthesia, including $40 \%$ major vascular surgery procedures. ${ }^{76}$ Atenolol was associated with a significant decrease in overall mortality and an increase in eventfree survival at 6 months, and this benefit was sustained for up to 2 years. The DECREASE trial selected 112 out of 1453 vascular surgery patients who combined at least one clinical risk factor and positive DSE, excluding patients with extensive wall motion abnormalities. ${ }^{9}$ Patients were randomized to standard care or bisoprolol, which was started at least 1 week before surgery and titrated according to heart rate. There was an $89 \%$ reduction in cardiac mortality and/or MI in the bisoprolol 
group $(3.4 \%$ vs. $34 \%, P<0.001)$, which was sustained for up to 3 years.

The PeriOperative Beta-BlockadE (POBBLE) trial included 103 low-risk patients undergoing elective infrarenal vascular surgery, randomized to metoprolol tartrate or placebo. ${ }^{74}$ The incidence of death, MI, or stroke at 30 days did not differ between the metoprolol and placebo groups (13 and 15\%, respectively, $P=0.78$ ). Patients were at low cardiac risk and those with a history of MI within the previous 2 years were excluded. In the Metoprolol after Vascular Surgery (MaVS) trial, 497 patients undergoing abdominal or infrainguinal vascular surgery were randomized to metoprolol succinate or placebo. ${ }^{77}$ The combined endpoint of death, MI, heart failure, arrhythmias, or stroke at 30 days did not differ between the metoprolol and placebo groups (10.2 and $12 \%$, respectively, $P=0.57)$. The Lee index was $\leq 2$ in $90 \%$ of patients and $\leq 1$ in $60 \%$.

The Diabetes Postoperative Mortality and Morbidity (DIPOM) trial selected 921 patients with diabetes, age $>39$ years, and a duration of surgery of $>1 \mathrm{~h}$ (39\% low-risk surgery). ${ }^{75}$ Patients were randomized to receive metoprolol succinate or placebo. The combined endpoint of death, MI, unstable angina, or heart failure at 30 days did not differ between the metoprolol and placebo groups (6 and $5 \%$, respectively, $P=0.66$ ). However, only $54 \%$ of the patients had a history of IHD, or an additional cardiac risk factor, and underwent high- or intermediate-risk surgery.

In the POISE trial, 8351 patients were randomized to metoprolol succinate or placebo. ${ }^{10}$ Patients were aged $\geq 45$ years and were included if they had known GVD, at least three out of seven clinical risk factors, or were scheduled for major vascular surgery. Treatment consisted of metoprolol succinate, $100 \mathrm{mg} 2-4 \mathrm{~h}$ prior to surgery, $100 \mathrm{mg}$ during the first $6 \mathrm{~h}$ after surgery, but withheld if systolic blood pressure dipped below $100 \mathrm{mmHg}$. Maintenance therapy was started $12 \mathrm{~h}$ later, bringing the total dose of metoprolol succinate in the first $24 \mathrm{~h}$ to $400 \mathrm{mg}$, at least in a number of patients. There was a $17 \%$ decrease in the composite endpoint, defined as death, MI, or non-fatal cardiac arrest at 30 days $(5.8 \%$ vs. $6.9 \%, P=0.04)$. However, the $30 \%$ decrease in non-fatal MI $(3.6 \%$ vs. $5.1 \%$, $P<0.001$ ) was partially offset by a $33 \%$ increase in total mortality $(3.1 \%$ vs. $2.3 \%, P=0.03)$ and a 2 -fold increase in stroke ( $1.0 \%$ vs. $0.5 \%, P=0.005)$. Hypotension was more frequent in patients receiving metoprolol $(15.0 \%$ vs. $9.7 \%$, $P<0.0001)$. Post hoc analysis showed that hypotension had the largest population-attributable risk for death and stroke.

Seven meta-analyses have pooled $5,11,6,15,8,22$ and 33 randomized published trials on perioperative $\beta$-blockers, totalling respectively $586,866,632,1077,2437,2057$, and 

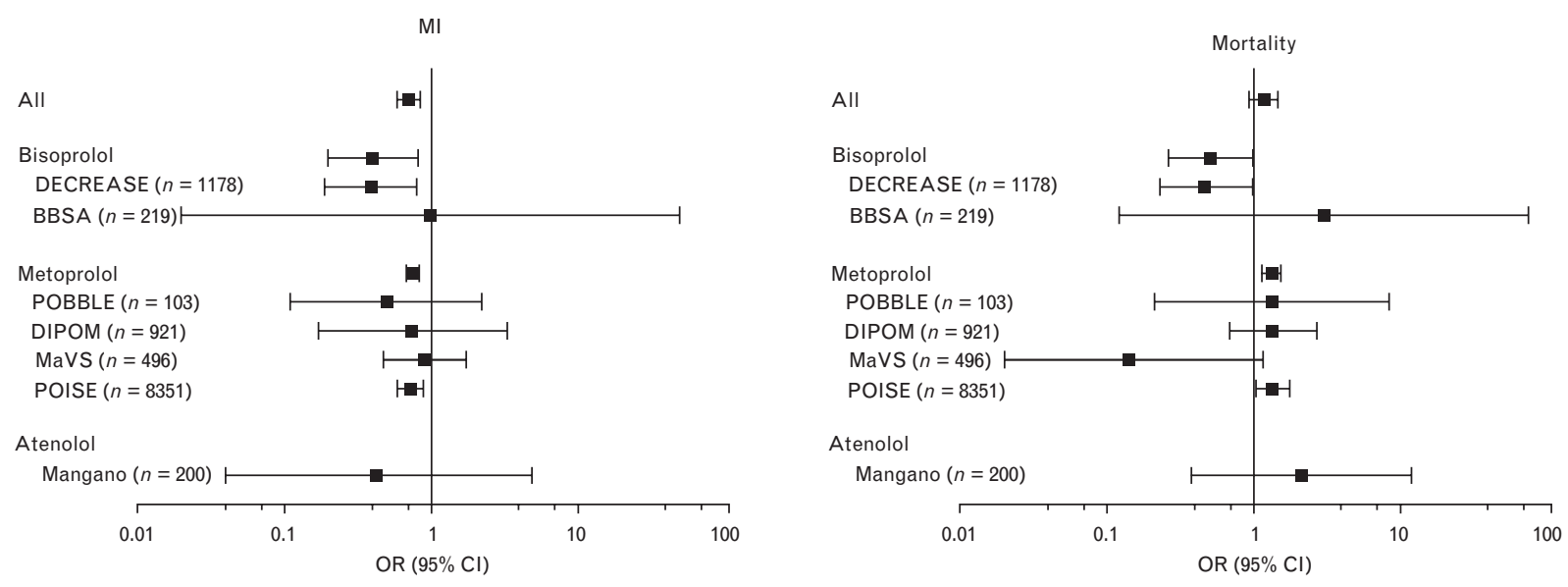

Effect of $\beta$-blockers on 30-day rates of non-fatal $\mathrm{Ml}$ and all-cause mortality as assessed from the seven randomized trials. Note: in the trial by Mangano et al., mortality was assessed at 6 months.

12306 patients. ${ }^{79-85}$ Five meta-analyses gave consistent results showing a significant reduction in perioperative myocardial ischaemia and MI in patients receiving $\beta$-blockers. ${ }^{79-83}$ These meta-analyses gave consistent results showing a significant reduction in perioperative myocardial ischaemia, MI, and cardiac mortality in patients receiving $\beta$-blockers. ${ }^{84,85}$ Risk reduction was more marked in high-risk patients. The most recent meta-analysis concluded that $\beta$-blockers result in 16 fewer non-fatal MIs per 1000 patients treated, but at the expense of three non-fatal disabling strokes and (possibly) three fatal cardiac or non-cardiac complications. ${ }^{83}$ However, it should be acknowledged that the recent POISE trial had the greatest weight in all of the above analyses. Indeed, $\sim 80 \%$ of the deaths, MIs, and strokes in this meta-analysis are derived from POISE, and this proportion was as high as $84 \%$ in the trials labelled low-bias risk. Hence, a more detailed analysis of the results of POISE compared with non-POISE trials is warranted (Table 7). First, in POISE, all-cause mortality was increased by $34 \%$ in patients receiving $\beta$-blockers; in the non-POISE trials the point estimate of treatment effect was consistent with a reduced, although not statistically significant, all-cause and cardiovascular mortality by $\beta$-blockers. The differential treatment effect seems to be caused by the high mortality in POISE patients who are given $\beta$-blockers $(3.1 \%$ vs. $1.9 \%$ in non-POISE trials), and not by differences in patients allocated to control therapy (2.3\% vs. $2.5 \%)$. Therefore, understanding of the cause and timing of deaths in POISE is important. Perioperative death in POISE patients allocated to metoprolol succinate was associated with perioperative hypotension, bradycardia, and stroke. A history of cerebrovascular disease was associated with an increased risk of stroke. Hypotension can be related to the use of a high dose of metoprolol without dose titration. It is considered that $200 \mathrm{mg}$ of metoprolol has approximately the same strength of $\beta$-blockade as $100 \mathrm{mg}$ of atenolol and $10 \mathrm{mg}$ of bisoprolol.

Discrepancies in the protective role of $\beta$-blockers can be explained by differences in patient characteristics, type of surgery, and the modalities of $\beta$-blockade (timing of onset, duration, dose titration, and type of drug). Also, these findings may be hampered by the inclusion of numerous trials which were not designed to assess the effect on perioperative cardiac risk or which used only a single $\beta$-blocker dose before anaesthesia without continuation after surgery. ${ }^{84} \mathrm{~A}$ recent meta-analysis suggested that most differences between trials on the cardioprotective effect of $\beta$-blockers could be attributed to the variability in heart rate response. ${ }^{86}$ In particular, the decrease in post-operative MI was highly significant when there was tight heart rate control.

Although observational studies should be interpreted with caution, they provide additional insights into the interactions between risk stratification and perioperative $\beta$-blockade.

In a prospective cohort comprising 1351 patients undergoing vascular surgery, $360(27 \%)$ were treated using $\beta$-blockers. ${ }^{63}$ In a study population of 1351 patients, $83 \%$ had $<3$ clinical risk factors. They experienced a lower risk of death or MI when using $\beta$-blockers $(0.8 \%)$ than without (2.3\%). In the $17 \%$ of patients who had $\geq 3$ risk factors, the risk of death or MI was reduced using $\beta$-blockers from 5.8 to $2.0 \%$ when stress-induced ischaemia was absent and from 33 to $2.8 \%$ when stress-induced ischaemia was limited (1-4 myocardial segments). Patients with extensive stress-induced ischaemia $(\geq 5 / 16$ myocardial segments) had a particularly high risk of death or MI whatever the treatment used (33\% with $\beta$-blockers 


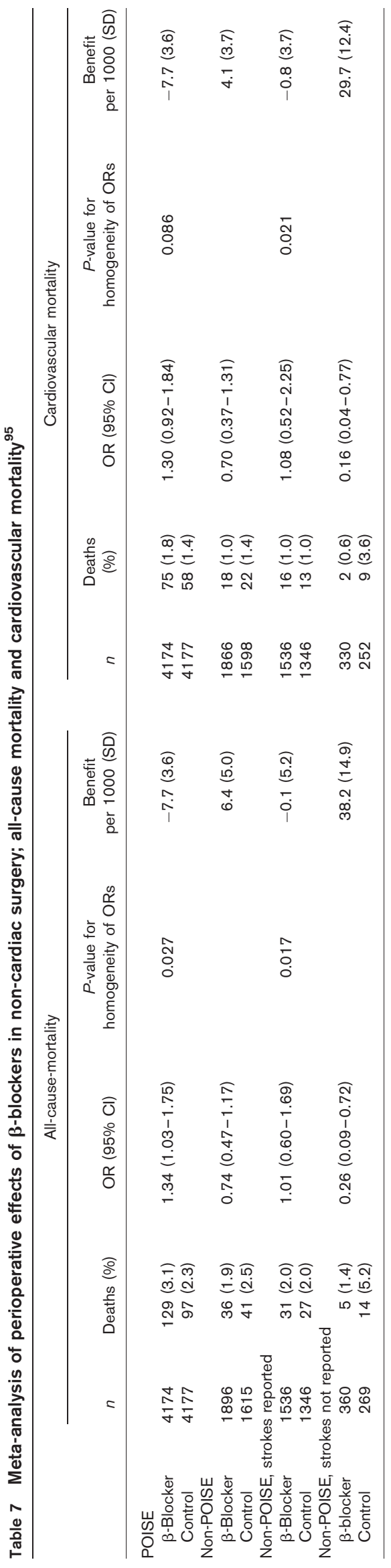

and $36 \%$ without). A large retrospective cohort drawn from a quality of care database analysed 663635 patients undergoing non-cardiac surgery (30\% high-risk surgery). ${ }^{87}$ The comparison of in-hospital mortality between 119632 patients receiving $\beta$-blockers and 216220 propensitymatched patients without $\beta$-blockers showed no difference overall (2.3\% vs. $2.4 \%$, respectively, $P=0.68$ ). However, there were marked differences according to patient risk profile. $\beta$-Blocker use was associated with a significant decrease in mortality when the Lee index was $\geq 3$. No significant difference was observed for a Lee index of 1 or 2. Mortality was increased in the lowest risk group (Lee index of 0 ).

Randomized trials selecting high-risk patients, cohort studies, and meta-analyses provide consistent evidence supporting a decrease in cardiac mortality and MI by $\beta$-blockers in patients with clinical risk factors undergoing high-risk (mainly vascular) surgery. Perioperative $\beta$-blockade is also cost-effective in these patients. However, patients with extensive ischaemia as demonstrated by stress testing are at particularly high risk of perioperative cardiac complications, despite perioperative $\beta$-blockers.

Conversely, randomized trials including low-risk patients and cohort studies suggest that perioperative $\beta$-blockade does not decrease the risk of cardiac complications in patients without clinical risk factors. The possibility of a harmful effect on mortality has been suggested by a retrospective cohort ${ }^{87}$ and the POISE trial. ${ }^{10}$ Bradycardia and hypotension may be harmful in patients with atherosclerosis, and possibly favour stroke.

This does not justify exposing low-risk patients to potential side effects in the absence of proven benefit. The issue remains debatable in intermediate-risk patients, i.e. those with one or two clinical risk factors. Results of the DECREASE-IV trial suggest that $\beta$-blockers should also be used in patients undergoing intermediate-risk surgery. ${ }^{88}$ Patients randomized to bisoprolol $(n=533)$ had a lower incidence of the primary efficacy endpoint than those randomized to bisoprolol-control therapy (2.1\% vs. $6.0 \%$ events, HR 0.34 , 95\% CI $0.17-0.67)$. An increased mortality following pre-operative $\beta$-blocker withdrawal has been reported in observational studies. ${ }^{89,90} \beta$-Blockers should be continued when prescribed for IHD or arrhythmias. When $\beta$-blockers are prescribed for hypertension, the absence of evidence in favour of a perioperative cardioprotective effect with other antihypertensive drugs does not support a change of therapy. $\beta$-Blockers should not be withdrawn in patients treated for stable heart failure due to $L V$ systolic dysfunction. In decompensated heart failure, $\beta$-blocker therapy may need to be reduced, or temporarily omitted. ${ }^{91}$ If possible, non-cardiac surgery should be deferred so that it can be performed under optimal medical therapy in a 
stable condition. Contra-indications to $\beta$-blockers (asthma, severe conduction disorders, symptomatic bradycardia, and symptomatic hypotension) should be respected. $\beta$-Blockers are not contra-indicated in patients with intermittent claudication, as in randomized trials, worsening of symptoms has not been shown to occur more frequently. ${ }^{92}$ Furthermore, a recent study showed that cardioselective $\beta$-blockers were associated with reduced mortality in patients with chronic obstructive pulmonary disease (COPD) undergoing vascular surgery. ${ }^{93}$ In the absence of contra-indications, $\beta$-blocker dose should be titrated to achieve a heart rate between 60 and 70 beats/min. $\beta_{1}$-Selective blockers without intrinsic sympathomimetic activity are favoured.

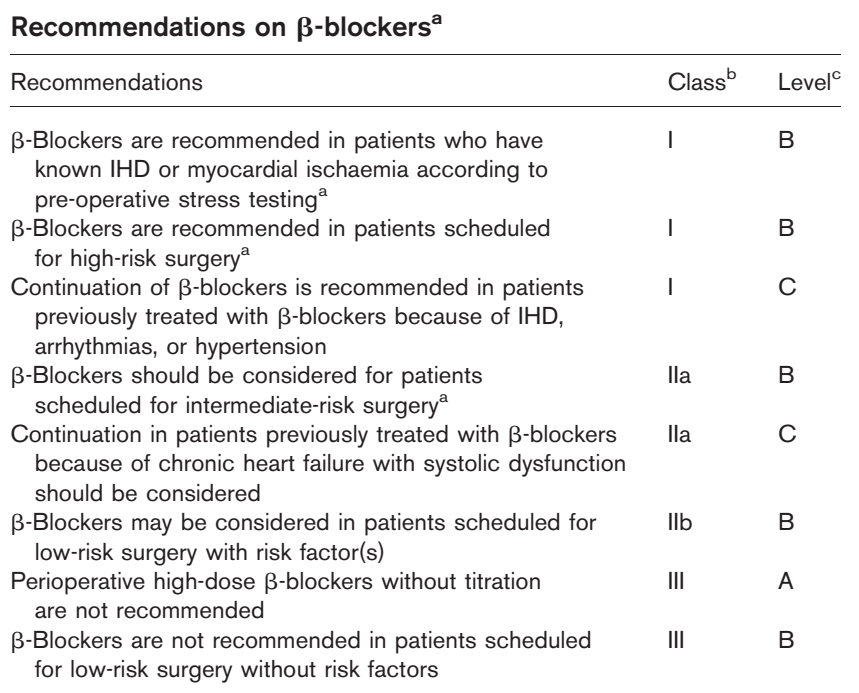

${ }^{a}$ Treatment should be initiated optimally between 30 days and at least 1 week before surgery. Target: heart rate $60-70$ beats $/ \mathrm{min}$, systolic blood pressure $>100 \mathrm{mmHg}$. ${ }^{b}$ Class of recommendation. ${ }^{\mathrm{C}}$ Level of evidence. IHD, ischaemic heart disease.

Treatment onset and the choice of the optimal dose of $\beta$-blockers are closely linked. Perioperative myocardial ischaemia and troponin release are reduced, and longterm outcome is improved, in patients who have a lower heart rate. ${ }^{94}$ On the other hand, bradycardia and hypotension should be avoided. This highlights the importance of preventing overtreatment with fixed high initial doses. The dose of $\beta$-blockers should be titrated, which requires that treatment be initiated optimally between 30 days and at least 1 week before surgery. It is recommended that treatment start with a daily dose of $2.5 \mathrm{mg}$ of bisoprolol or $50 \mathrm{mg}$ of metoprolol succinate which should then be adjusted before surgery to achieve a resting heart rate of between 60 and 70 beats/min with systolic blood pressure $>100 \mathrm{mmHg}$. The goal for heart rate is the same during the whole perioperative period, using i.v. administration when oral administration is not possible. Post-operative tachycardia should result in the first instance in the treatment of the underlying cause, for example hypovolaemia, pain, blood loss, or infection, rather than the $\beta$-blocker dose simply being increased.

The optimal duration of perioperative $\beta$-blocker therapy cannot be derived from randomized trials. The occurrence of delayed cardiac events is an incentive to continue $\beta$-blocker therapy for at least several months. Longterm $\beta$-blocker therapy should be used in patients who had a positive pre-operative stress test. Current concepts of cardioprotection have led to recommendations to use selective $\beta_{1}$-blockers without intrinsic sympathomimetic activity and with a long half-life, e.g. bisoprolol.

\section{Statins}

3-Hydroxy-3-methylglutaryl co-enzyme A reductase inhibitors (statins) are widely prescribed in patients with or at risk of IHD because of their lipid-lowering effect. Patients with non-coronary atherosclerosis (carotid, peripheral, aortic, renal) should receive statin therapy for secondary prevention, independently of non-cardiac surgery. ${ }^{96}$ Statins also induce coronary plaque stabilization by decreasing lipid oxidation, inflammation, matrix metalloproteinase, and cell death, and by increasing tissue inhibitor of metalloproteinase and collagen. These so-called non-lipid or pleiotropic effects may prevent plaque rupture and subsequent $\mathrm{MI}$ in the perioperative period. $^{97}$

Multiple large clinical trials and observational studies have demonstrated a beneficial effect of perioperative statin use. ${ }^{98,99}$ In the first prospective, randomized controlled trial, 100 patients scheduled for vascular surgery were allocated to $20 \mathrm{mg}$ of atorvastatin or placebo once a day for 45 days, irrespective of their serum cholesterol concentration. ${ }^{100}$ Vascular surgery was performed on average 31 days after randomization, and patients were followed-up over 6 months. During these 6 months of follow-up, atorvastatin significantly reduced the incidence of cardiac events ( $8 \%$ vs. $26 \%, P=0.03$ ). A meta-analysis of 223010 patients from 12 retrospective and three prospective trials showed that statins reduced mortality significantly by $44 \%$ in non-cardiac surgery and by $59 \%$ in vascular surgery. ${ }^{98}$ The most recent randomized controlled trial was the DECREASE-III study. A total of 497 vascular surgery patients were allocated to either fluvastatin (extended release $80 \mathrm{mg}$ once daily) or placebo, starting 37 days prior to surgery. The incidence of myocardial ischaemia in patients allocated to fluvastatin or placebo was $10.8 \%$ vs. $19.0 \%$, respectively (OR $0.55,95 \%$ CI $0.34-0.88)$. The incidence of cardiac death or MI in the two study groups was $4.8 \%$ vs. $10.2 \%$, respectively (OR $0.47,95 \%$ CI $0.24-0.94) .{ }^{101}$

A concern related to the use of perioperative statin therapy has been the risk of statin-induced myopathy and rhabdomyolysis. Perioperatively, factors increasing the risk of statin-induced myopathy are numerous, e.g. 
the impairment of renal function after major surgery, and multiple drug use during anaesthesia. Furthermore, the use of analgesic drugs and post-operative pain may mask signs of myopathy. Failure to detect statin-induced myopathy may then lead to the statin being continued and the subsequent development of rhabdomyolysis and acute renal failure. However, no studies have been published that support this concern, except for some case reports. In a retrospective study of 981 consecutive patients undergoing vascular surgery, no cases of rhabdomyolysis, significantly higher creatine kinase level, or increased incidence of myopathy were observed in statin users. ${ }^{102}$

Recently it has been suggested that discontinuation of statins may cause a rebound effect and be disadvantageous. ${ }^{99,103}$ A potential limitation of perioperative statin use is the lack of an i.v. formulation.

Therefore, statins with a long half-life or extended release formulations such as rosuvastatin, atorvastatin, and fluvastatin extended release are recommended, to bridge the period immediately after surgery when oral intake is not feasible.

\section{Recommendations on statins}

\begin{tabular}{lll}
\hline Recommendations & Class $^{\mathrm{a}}$ & Level $^{\mathrm{b}}$ \\
\hline It is recommended that statins be started in & । & B \\
high-risk surgery patients, optimally between & & \\
30 days and at least 1 week before surgery & I & C \\
It is recommended that statins be continued \\
perioperatively
\end{tabular}

${ }^{a}$ Class of recommendation. ${ }^{b}$ Level of evidence.

\section{Nitrates}

Nitroglycerin is well known to reverse myocardial ischaemia. One small but controlled study has demonstrated decreased perioperative myocardial ischaemia in patients with stable angina given i.v. nitroglycerin during noncardiac surgery. ${ }^{104}$ However, no effect was observed on the incidence of MI or cardiac death. These observations were confirmed in a similar study, showing no effect on either myocardial ischaemia, MI, or cardiac death. ${ }^{105}$ Furthermore, perioperative use of nitroglycerin may pose a significant haemodynamic risk to the patients. Decreased preload may lead to tachycardia, and hypotension.

\section{Recommendations on nitrates}

\begin{tabular}{lll}
\hline Recommendations & Class $^{\mathrm{a}}$ & Level $^{\mathrm{b}}$ \\
\hline $\begin{array}{l}\text { Perioperative nitroglycerin use for the prevention of } \\
\text { adverse ischaemic events may be considered }\end{array}$ & llb & $\mathrm{B}$ \\
\hline${ }^{\mathrm{a}}$ Class of recommendation. ${ }^{\mathrm{b}}$ Level of evidence. & & \\
\hline
\end{tabular}

\section{Angiotensin-converting enzyme inhibitors}

Independently of the blood pressure-lowering effect, angiotensin-converting enzyme (ACE) inhibitors pre- serve organ function. This effect is related to improvement of endothelial function, anti-inflammatory properties, and a direct interference with atherogenesis. ${ }^{106}$ The inhibition of ACE may prevent events related to myocardial ischaemia and $\mathrm{LV}$ dysfunction. Therefore, it seems reasonable to suggest that perioperative treatment with ACE inhibitors may have beneficial effects on postoperative outcome.

The Quinapril on Vascular ACE and Determinants of Ischemia (QUO VADIS) study compared the effect of the ACE inhibitors quinapril with that of placebo in patients undergoing cardiac surgery. Quinapril treatment was started 4 weeks before elective surgery and was continued up to 1 year after surgery. ${ }^{107}$ This trial demonstrated that post-operative cardiovascular events were significantly reduced (HR $0.23,95 \%$ CI $0.06-0.87$ ) in patients treated with quinapril. The beneficial effect in the QUO VADIS study, however, could be the result of the postoperative treatment. A recent review provided conflicting data concerning ACE inhibitors after cardiac surgery. ${ }^{108}$

Additionally, perioperative use of ACE inhibitors carries a risk of severe hypotension under anaesthesia, in particular following induction and concomitant $\beta$-blocker use. Нypotension is less frequent when ACE inhibitors are discontinued the day before surgery. Although this remains debated, ACE inhibitor withdrawal may be considered $24 \mathrm{~h}$ before surgery when they are prescribed for hypertension. They should be resumed after surgery as soon as volume is stable. The risk of hypotension is at least as high with angiotensin receptor blockers (ARBs) as with ACE inhibitors, and the response to vasopressors may be impaired. In patients with LV systolic dysfunction who are in a stable clinical condition, it seems reasonable to continue ACE inhibitors during the perioperative period under close monitoring. When LV dysfunction is discovered during pre-operative evaluation in untreated patients in stable condition, surgery should be postponed, if possible, to introduce ACE inhibitors and $\beta$-blockers as recommended by the ESC Guidelines on heart failure. ${ }^{91}$

\begin{tabular}{|c|c|c|}
\hline Recommendations & Class $^{a}$ & Level $^{\mathrm{b}}$ \\
\hline $\begin{array}{l}\text { It is recommended that ACE inhibitors be continued } \\
\text { during non-cardiac surgery in stable patients with } \\
\text { LV systolic dysfunction }\end{array}$ & 1 & C \\
\hline $\begin{array}{l}\text { ACE inhibitors are recommended in cardiac-stable } \\
\text { patients with LV systolic dysfunction scheduled } \\
\text { for high-risk surgery }\end{array}$ & 1 & C \\
\hline $\begin{array}{l}\text { ACE inhibitors should be considered in cardiac-stable } \\
\text { patients with LV systolic dysfunction scheduled } \\
\text { for low-/intermediate-risk surgery }\end{array}$ & Ila & C \\
\hline $\begin{array}{l}\text { Transient discontinuation of ACE inhibitors before } \\
\text { non-cardiac surgery in hypertensive patients } \\
\text { should be considered }\end{array}$ & Ila & C \\
\hline
\end{tabular}

$\mathrm{ACE}$, angiotensin-converting enzyme; LV, left ventricular. ${ }^{a}$ Class of recommendation. ${ }^{b}$ Level of evidence. 


\section{Calcium channel blockers}

The effect of calcium channel blockers on the balance between myocardial oxygen supply and demand makes them theoretically suitable for risk reduction strategies. It is necessary to distinguish between dihydropyridines that do not act directly on heart rate and diltiazem or verapamil that lower the heart rate.

The relevance of randomized trials assessing the perioperative effect of calcium channel blockers is limited by their small size, the lack of risk stratification, and the absence of the systematic reporting of cardiac death and MI. A meta-analysis pooled 11 randomized trials totalling 1007 patients. All patients underwent non-cardiac surgery under calcium channel blockers (diltiazem in seven trials, verapamil in two, and nifedipine in one, and one other trial incorporated three arms: control, diltiazem, and nifedipine). ${ }^{109}$ There was a significant reduction in the number of episodes of myocardial ischaemia and supraventricular tachycardia (SVT) in the pooled analyses on calcium channel blockers. However, the decrease in mortality and MI reached statistical significance only when both endpoints were combined in a composite endpoint of death and/or MI (relative risk 0.35 , 95\% CI $0.08-0.83, P=0.02$ ). Subgroup analyses favoured diltiazem. Another study in 1000 patients having acute or elective aortic aneurysm surgery showed that dihydropyridine calcium channel blocker use was independently associated with an increased incidence of perioperative mortality. ${ }^{110}$ The use of short-acting dihydropyridines, in particular nifedipine capsules, should be avoided.

Thus, although heart rate-reducing calcium channel blockers are not indicated in patients with heart failure and systolic dysfunction, in patients who have contraindications to $\beta$-blockers the continuation or the introduction of heart rate-reducing calcium channel blockers may be considered.

\begin{tabular}{lll} 
Recommendations on calcium channel blockers & & \\
\hline Recommendations & Class $^{\mathrm{a}}$ & Level $^{\mathrm{b}}$ \\
\hline $\begin{array}{l}\text { It is recommended that calcium channel blockers be } \\
\text { continued during non-cardiac surgery in patients } \\
\text { with Prinzmetal angina pectoris }\end{array}$ & $\mathrm{C}$ \\
$\begin{array}{l}\text { Heart rate-reducing calcium channel blockers, in } \\
\text { particular diltiazem, may be considered before } \\
\text { non-cardiac surgery in patients who have contra- } \\
\text { indications to } \beta \text {-blockers }\end{array}$ & Clb \\
$\begin{array}{l}\text { Routine use of calcium channel blockers to } \\
\text { reduce the risk of perioperative cardiovascular }\end{array}$ & III \\
complications is not recommended & \\
\hline
\end{tabular}

${ }^{a}$ Class of recommendation. ${ }^{b}$ Level of evidence.

\section{Ivabradine}

Ivabradine is a specific inhibitor of the pacemaker in the sino-atrial node and reduces heart rate independently of sympathetic activation. It does not affect blood pressure or myocardial contractility. In a randomized trial of
111 vascular surgery patients, both ivabradine and metoprolol succinate reduced the incidence of ischaemia and MI significantly when compared with placebo. These preliminary findings need to be confirmed by future studies; ivabradine might be considered for patients with strict contra-indications to $\beta$-blockers. ${ }^{111}$

\section{$\alpha_{2}$ Receptor agonists}

$\alpha_{2}$ Receptor agonists reduce post-ganglionic noradrenaline output and therefore might reduce the catecholamine surge during surgery. The European Mivazerol trial randomized 1897 patients with IHD who underwent intermediate- or high-risk non-cardiac surgery. ${ }^{112}$ Mivazerol did not decrease the incidence of death or MI in the whole population. However, there was a reduction of post-operative death or MI observed in a subpopulation of 904 vascular surgery patients. A more recent study including 190 patients with clinical risk factors or IHD showed a decrease in 30-day and 2-year mortality after perioperative use of clonidine. ${ }^{113}$ However, there was no decrease in MI. A meta-analysis pooled 23 randomized trials, which included cardiac surgery in 10, vascular surgery in eight, and non-vascular surgery in three cases. ${ }^{114}$

Perioperative use of $\alpha_{2}$ receptor agonists was associated with a decrease in mortality and MI only in the subgroup having vascular surgery, while there was no benefit in non-vascular surgery.

\section{Recommendations on $\alpha_{2}$ receptor agonists}

\begin{tabular}{llc}
\hline Recommendations & Class $^{\mathrm{a}}$ & Level $^{\mathrm{b}}$ \\
\hline $\begin{array}{l}\alpha_{2} \text { Receptor agonists may be considered to } \\
\text { reduce the risk of perioperative cardiovascular } \\
\text { complications in vascular surgery patients }\end{array}$ & $\mathrm{llb}$ & $\mathrm{B}$ \\
\hline${ }^{\mathrm{a}}$ Class of recommendation. ${ }^{\mathrm{b}}$ Level of evidence. & &
\end{tabular}

\section{Diuretics}

Diuretics are a frequent pharmacological treatment in patients with hypertension or heart failure as underlying diseases. In hypertension, diuretics are usually used at low dose with relatively moderate blood pressurelowering effect. In general, diuretics for hypertension can be discontinued on the day of surgery, and resumed orally when possible. If blood pressure reduction is required before oral therapy can be continued, other antihypertensive agents given i.v. may be preferred. In heart failure, diuretics are often used at high dose. Dosage increase should be considered if signs of fluid retention are present. Dosage reduction should be considered if there is risk of hypovolaemia, hypotension, and electrolyte disturbances. In general, diuretic treatment, if necessary to control heart failure, should be continued up to the day of surgery, and resumed orally when possible. In the perioperative period, volume status in patients 
with heart failure should be carefully monitored and loop diuretics may be given i.v. to control volume overload.

In any patient given diuretics, the possibility of electrolyte disturbance should be considered, as diuretics increase renal excretion of $\mathrm{K}$ and $\mathrm{Mg}$. Hypokalaemia is reported to occur in up to $34 \%$ of patients undergoing surgery (mostly non-cardiac). ${ }^{115}$ Hypokalaemia is well known to increase significantly the risk of ventricular tachycardia (VT) and ventricular fibrillation in cardiac disease. ${ }^{116}$ In a study of 688 patients with cardiac disease undergoing non-cardiac surgery, hypokalaemia was independently associated with perioperative mortality. ${ }^{117} \mathrm{On}$ the other hand, in a study of 150 patients undergoing noncardiac surgery, no increase in intraoperative arrhythmias was observed with hypokalaemia. ${ }^{115}$ However, this latter study was relatively small and most patients had no evidence of cardiac disease. Significantly, the use of K and $\mathrm{Mg}$-sparing diuretics, i.e. aldosterone antagonists (spironolactone and eplerenone), is now well known to reduce mortality in severe heart failure. ${ }^{118}$ In general, $\mathrm{K}$ and $\mathrm{Mg}$ homeostasis should be evaluated pre-operatively. Special attention should be given to patients on diuretics and patients prone to develop arrhythmia. Any electrolyte disturbance-especially hypokalaemia and hypomagnesaemia - should be corrected in due time before surgery. Dietary advice to increase intake of $\mathrm{K}$ and $\mathrm{Mg}$ should be given; depleting drugs should, if possible, be reduced; sparing diuretics may be added or preferred; and supplementation may be given. Acute pre-operative repletion in asymptomatic patients may be associated with more risks than benefits. Thus, minor, asymptomatic electrolyte disturbances should not delay acute surgery.

\begin{tabular}{|c|c|c|}
\hline Recommendations & Class $^{a}$ & Level $^{b}$ \\
\hline $\begin{array}{l}\text { It is recommended that electrolyte disturbances be } \\
\text { corrected before surgery }\end{array}$ & 1 & B \\
\hline $\begin{array}{l}\text { It is recommended that hypertensive patients discontinue } \\
\text { low-dose diuretics on the day of surgery and resume } \\
\text { orally when possible }\end{array}$ & I & C \\
\hline $\begin{array}{l}\text { It is recommended that diuretics be continued in heart } \\
\text { failure patients up to the day of surgery, resumed } \\
\text { intravenously perioperatively, and continued orally } \\
\text { when possible }\end{array}$ & I & C \\
\hline
\end{tabular}

${ }^{\mathrm{a}}$ Class of recommendation. ${ }^{\mathrm{b}}$ Level of evidence.

\section{Aspirin}

Though aspirin is widely used in patients with IHD and especially after coronary stent placement, the evidence of aspirin in the perioperative period setting is limited. In a randomized trial of 232 patients undergoing carotid endarterectomy, aspirin was shown to be effective in preventing intraoperative and post-operative stroke, though no effect on death or MI was noted. ${ }^{119}$ A metaanalysis in 2001 demonstrated a reduction in serious vascular events and vascular death in vascular surgery patients. ${ }^{120}$ This study included 10 trials of antiplatelet treatment in lower limb bypass surgery of which six involved aspirin treatment. However, the benefit of antiplatelet therapy did not reach statistical significance for the combined endpoint of vascular events $(\mathrm{OR}=0.8$, $95 \%$ CI $0.5-1.1)$ in this vascular surgery population.

Concerns of promoting perioperative haemorrhagic complications often led to the discontinuation of aspirin in the perioperative period. A large meta-analysis, including 41 studies in 49590 patients, which compared perioprocedural withdrawal vs. bleeding risks of aspirin, concluded that the risk of bleeding complications was increased by 1.5 but that aspirin did not lead to higher severity levels of bleeding complications. ${ }^{121}$ A systematic review in subjects at risk of or with IHD demonstrated that aspirin non-adherence/withdrawal was associated with a 3 -fold higher risk of major adverse cardiac events $(\mathrm{OR}=3.14$, 95\% CI 1.8-5.6). ${ }^{122}$ Aspirin should only be discontinued if the bleeding risk outweighs the potential cardiac benefit. Prior to minor surgical or endoscopic procedures, a careful consideration should be given to the question of withdrawing antithrombotic medications. In principle and based on individualized 'risk to benefit' assessments, there is often no need for stopping the anti-platelet treatment prior to the aforementioned procedures in patients who are taking antiplatelet medications. For patients receiving antiplatelet therapy, i.e. aspirin, clopidogrel, or both, with excessive or life-threatening perioperative bleeding, transfusion of platelets or administration of other prohaemostatic agents is recommended.

\begin{tabular}{lll} 
Recommendations on aspirin & & \\
\hline Recommendations & Class $^{\mathrm{a}}$ & Level $^{\mathrm{b}}$ \\
\hline $\begin{array}{l}\text { Continuation of aspirin in patients previously treated with } \\
\text { aspirin should be considered in the perioperative period }\end{array}$ & $\mathrm{Bla}$ & $\mathrm{B}$ \\
$\begin{array}{l}\text { Discontinuation of aspirin therapy in patients previously } \\
\text { treated with aspirin should be considered only in those } \\
\text { in whom haemostasis is difficult to control during surgery }\end{array}$ & $\mathrm{B}$ \\
\hline${ }^{\mathrm{a}}$ Class of recommendation. ${ }^{\mathrm{b}}$ Level of evidence. &
\end{tabular}

\section{Anticoagulant therapy}

Anticoagulant therapy is associated with increased bleeding during non-cardiac surgery. In some patients, this risk will be outweighed by the benefit of anticoagulant therapy, and drug therapy should be maintained or modified, whereas in other patients with low risk of thrombosis, therapy should be stopped in order to minimize bleeding complications.

Patients treated with oral anticoagulant therapy with vitamin $\mathrm{K}$ antagonists (VKAs) have an increased risk of periprocedural and post-procedural bleeding. If the international normalized ratio (INR) is $<1.5$, surgery can be performed safely (Table 8 ). However, in patients with a high risk of thromboembolism, discontinuation of VKAs 
Table 8 Bridging therapy of VKA with UFH or LMWH in high- and low-risk patients/procedures ${ }^{125}$

Low thromboembolic risk/low bleeding risk

Continue anticoagulant therapy with INR in therapeutic range.

Low thromboembolic risk/high bleeding risk

Discontinue anticoagulant therapy 5 days before the procedure.

Start LMWH prophylaxis once daily or UFH i.v.1 day after acenocoumaro interruption, and 2 days after warfarin interruption. Administer the last dose of LMWH at least $12 \mathrm{~h}$ before the procedure or give UFH i.v. up to $4 \mathrm{~h}$ prior to surgery.

Resume LMWH or UFH at the pre-procedural dose 1-2 days (at least $12 \mathrm{~h}$ ) after the procedure according to haemostatic status. Resume anticoagulant therapy 1 to 2 days after surgery at the pre-procedural dose $+50 \%$ boost dose for two consecutive days according to the haemostatic status.

LMWH or UFH is continued until the INR has returned to therapeutic levels.

High thromboembolic risk

Discontinue anticoagulant therapy 5 days before the procedure.

Start therapeutic LMWH twice daily or UFH i.v. 1 day after acenocoumarol interruption, and 2 days after warfarin interruption. Administer the last dose of LMWH at least $12 \mathrm{~h}$ before the procedure or give UFH i.v. up to $4 \mathrm{~h}$ prior to surgery.

Resume LMWH or UFH at the pre-procedural dose 1-2 days (at least $12 \mathrm{~h}$ ) after the procedure according to haemostatic status. Resume anticoagulant therapy 1-2 days after surgery at the pre-procedural dose $+50 \%$ boost dose for two consecutive days according to haemostatic status.

$\mathrm{LMWH}$ or UFH is continued until the INR has returned to therapeutic levels.

INR, international normalized ratio; LMWH, low molecular weight heparin; UFH, unfractionated heparin.

is hazardous and these patients will need bridging therapy with unfractionated heparin (UFH) or therapeutic-dose low molecular weight heparin (LMWH) i.v. or s.c. ${ }^{123-125} \mathrm{~A}$ high thromboembolic risk is present among other conditions, in patients with atrial fibrillation (AF), mechanical prosthetic heart valves, biological prosthetic heart valves or mitral valvular repair within the last 3 months, or recent venous thromboembolism $(<3$ months) plus thrombophilia. Bridging therapy is now most often performed with therapeutic-dose s.c. LMWH. VKAs are stopped 5 days (i.e. five doses of VKA) prior to surgery; LMWH or UFH are started 1 day after acenocoumarol interruption, and 2 days after warfarin interruption. In high thromboembolic risk patients, $70 \mathrm{U} / \mathrm{kg}$ of antifactor Xa twice daily are recommended and prophylactic once-daily doses in low-risk patients (Table 9). ${ }^{126}$ The last dose of LMWH should be administered at least $12 \mathrm{~h}$ before the procedure. In patients with mechanical prosthetic heart valves, the evidence for i.v. UFH is more solid. Thus, in some centres these patients are hospitalized and treated with i.v. UFHs up until $4 \mathrm{~h}$ prior to surgery, and treatment with
UFH is resumed after surgery until the INR is in the therapeutic range. ${ }^{124}$ On the day of the procedure, the INR is checked.

Consideration should be given to postponing the procedure if the INR is $>1.5$. LMWH or UFH is resumed at the pre-procedural dose 1-2 days after surgery, depending on the haemostatic status, but at least $12 \mathrm{~h}$ after the procedure. Oral anticoagulants should be resumed on day 1 or 2 after surgery depending on haemostasis sufficiency (if the patient can take oral therapy) at the pre-operative maintenance dose plus a boost dose of $50 \%$ for two consecutive days; the maintenance dose should be administered thereafter. LMWH or UFH should be continued until the INR returns to therapeutic levels.

Furthermore, the type of surgical procedure should be taken into consideration, as the bleeding risk varies considerably and affects the ability to ensure haemostatic control. Procedures with a high risk of serious bleeding complications are those where compression cannot be performed. In these cases, discontinuation of oral anticoagulants and bridging therapy with LMWH are warranted. In patients undergoing surgery with a low risk of serious bleeding, such as cataract surgery, no changes in oral anticoagulation therapy are needed.

In patients who are receiving VKAs and require reversal of the anticoagulant effect for an urgent surgical procedure, low-dose $(2.5-5.0 \mathrm{mg})$ i.v. or oral vitamin $\mathrm{K}$ is recommended. For more immediate reversal of the anticoagulant effect of VKAs, treatment with fresh-frozen plasma or another prothrombin concentrate in addition to low-dose i.v. or oral vitamin $\mathrm{K}$ is recommended. In patients receiving UFH and requiring reversal of the anticoagulant effect for an urgent surgical procedure, cessation of therapy is enough. When given as an infusion, the anticoagulant effect of UFH reaches steady state within 4-6 h. So on cessation of an infusion, coagulation should be mostly normal after $4 \mathrm{~h}$. When UFH is given s.c., the anticoagulant effect is more prolonged. For immediate reversal, the antidote is protamine sulfate. However, protamine sulfate can potentially provoke anaphylactic reactions with cardiovascular collapse, especially if infused too quickly. The dose of protamine sulfate can be calculated by the assessment of the amount of

Table 9 Anticoagulation protocols applied according to patient thromboembolic risk ${ }^{126}$

\begin{tabular}{|c|c|c|c|c|}
\hline \multirow[b]{2}{*}{ Weight (kg) } & \multicolumn{2}{|c|}{ Patient at high thromboembolic risk } & \multicolumn{2}{|c|}{ Patient at low thromboembolic risk } \\
\hline & Nadroparin (twice daily, s.c.) (IU) & Enoxaparin (twice daily, s.c.) (IU) & Enoxaparin (once daily, s.c.) (IU) & Enoxaparin (twice daily, s.c.) (IU) \\
\hline$<50$ & 2850 & 2000 & 2850 & 4000 \\
\hline $50-69$ & 3800 & 4000 & 3800 & 4000 \\
\hline $70-89$ & 5700 & 6000 & 5700 & 4000 \\
\hline $90-110$ & 7600 & 8000 & 5700 & 4000 \\
\hline$>110$ & 9500 & 10000 & 5700 & 4000 \\
\hline
\end{tabular}

IU, international units; LMWH, low molecular weight heparin; s.c., subcutaneous. 
heparin received in the previous $2 \mathrm{~h}$. The dose of protamine sulfate for reversal for a heparin infusion then is $1 \mathrm{mg}$ per $100 \mathrm{U}$ of heparin sodium. If the heparin infusion was stopped for $>30$ min but $<2 \mathrm{~h}$, then use half the dose of protamine sulfate; if the heparin infusion was stopped for $>2 \mathrm{~h}$ but $<4 \mathrm{~h}$, then use a quarter of the dose. The maximum dose of protamine sulfate is $50 \mathrm{mg}$. In patients who are receiving LMWH the anticoagulant effect may be reversed within $8 \mathrm{~h}$ of the last dose because of the short half-life. If immediate reversal is required, i.v. protamine sulfate can be used, but anti-Xa activity is never completely neutralized (maximum of $60-75 \%$ ).

A summary of the recommended way to minimize bleeding and thromboembolic events during surgery is given in Table 8.

\section{Revascularization}

The main objective of prophylactic myocardial revascularization is the prevention of potentially lethal perioperative MI. While revascularization may be particularly effective in treating high-grade stenoses, it cannot prevent rupture of vulnerable plaques during the stress of surgery. The latter mechanism has been advocated in at least half of fatal cases of perioperative MI and may explain the lack of specificity of stress imaging techniques in predicting infarct-related coronary artery lesions. ${ }^{37,127}$

Patients who are clinically stable in the years after coronary artery bypass grafting $(\mathrm{CABG})$ have a diminished risk of cardiac complications after subsequent non-cardiac surgery. Data from the Coronary Artery Surgery Study (CASS) registry indicate that this is particularly the case in patients with triple vessel disease and/or depressed LV function but also in the case of high-risk surgery. ${ }^{128}$ Therefore, patients who had CABG within the previous 5 years can be sent for surgery, if their clinical condition has remained unchanged since their last examination.

Patients with previous percutaneous revascularization may be at higher risk of cardiac events during or after subsequent non-cardiac surgery, particularly in cases of unplanned or urgent surgery after coronary stenting. After the introduction of angioplasty, it seemed that conventional percutaneous coronary intervention (PCI) did not worsen outcomes after surgery, even if performed as early as 11 days after PCI. ${ }^{129}$ The advent of stenting in the mid-1990s dramatically changed the scenario. Indeed, extremely high mortality rates (up to 20\%) were reported in relation to acute stent thrombosis at the time of surgery if performed within weeks after coronary stenting with discontinuation of anti-platelet therapy. ${ }^{130,131}$ Therefore, it is preferred that elective surgery be postponed for a minimum period of 6 weeks and optimally up to 3 months after bare metal stent implantation and that dual antiplatelet therapy be continued. When surgery was performed within this period, discontinuation of dual antiplatelet therapy was associated with an increased incidence of stent thrombosis. ${ }^{130,131}$ After 3 months, patients can be sent for non-cardiac surgery, with continuation of at least aspirin therapy ${ }^{132}$ (Fig. 3).

In 2002, DESs were introduced in Europe and became widely accepted as an efficient tool to reduce in-stent restenosis further. However, their major drawback is the need for prolonged dual antiplatelet therapy by aspirin and clopidogrel for at least 12 months. When surgery was performed within this period, discontinuation of dual

Fig. 3

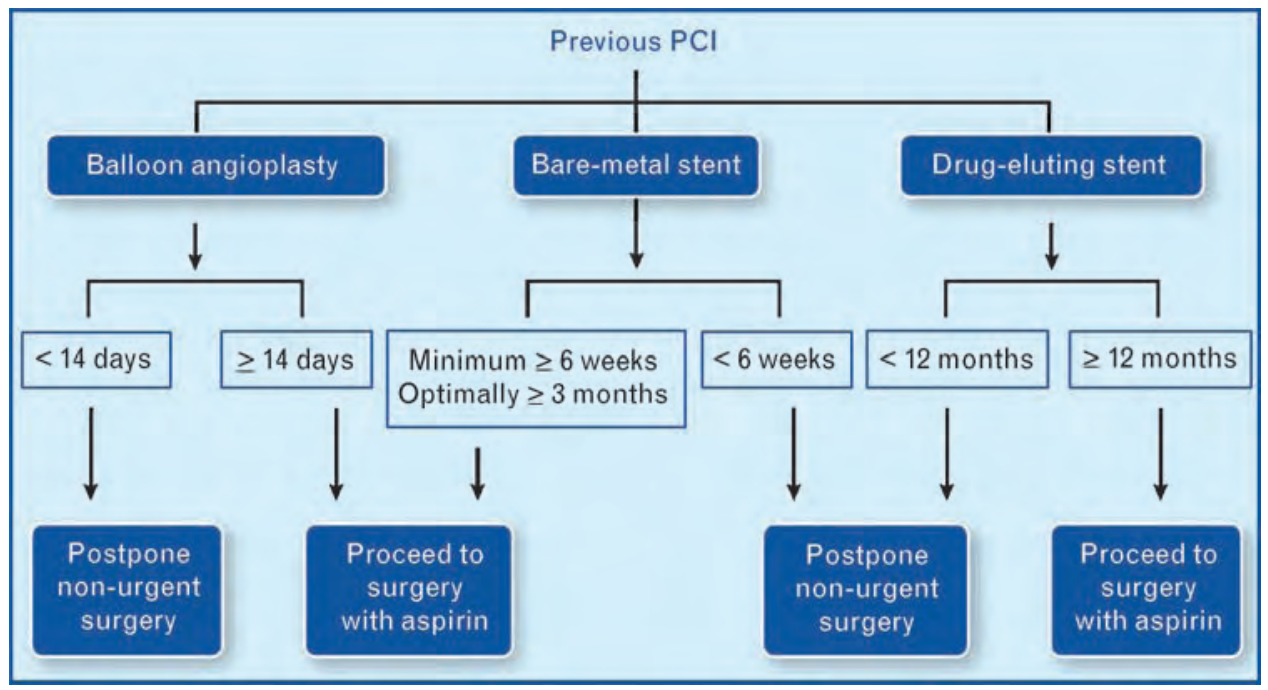

Recommendations for timing of non-cardiac surgery after $\mathrm{PCl}{ }^{133} \mathrm{PCl}$, percutaneous coronary intervention. 
antiplatelet therapy was associated with an increased incidence of stent thrombosis. It is now generally accepted that after DES implantation, elective surgery should not take place until after at least 12 months of continuous dual antiplatelet therapy ${ }^{133}$ (Fig. 3). After 12 months, patients can be sent for non-cardiac surgery, with continuation of at least aspirin therapy. The need for surgery in relation to its timing and the specific pathology (e.g. malignant tumour, vascular aneurysm repair) should be balanced against the excessive risk of stent thrombosis during the first year following DES implantation and a careful 'case-by-case' consideration is advisable. Discussion between the surgeon, the anaesthesiologist, and the treating cardiologist about this matter is recommended in order to achieve a reasonable expert consensus.

In patients who require temporary interruption of aspirinor clopidogrel-containing drugs before surgery or a procedure it is recommended that this treatment be stopped at least 5 days and, preferably as much as 10 days, prior to the procedure. Therapy can be resumed after $\sim 24 \mathrm{~h}$ (or the next morning) after surgery when there is adequate haemostasis. In patients in need of an urgent surgical or other invasive procedure, with potential excessive or lifethreatening perioperative bleeding, transfusion of platelets or administration of other prohaemostatic agents is recommended. ${ }^{134}$

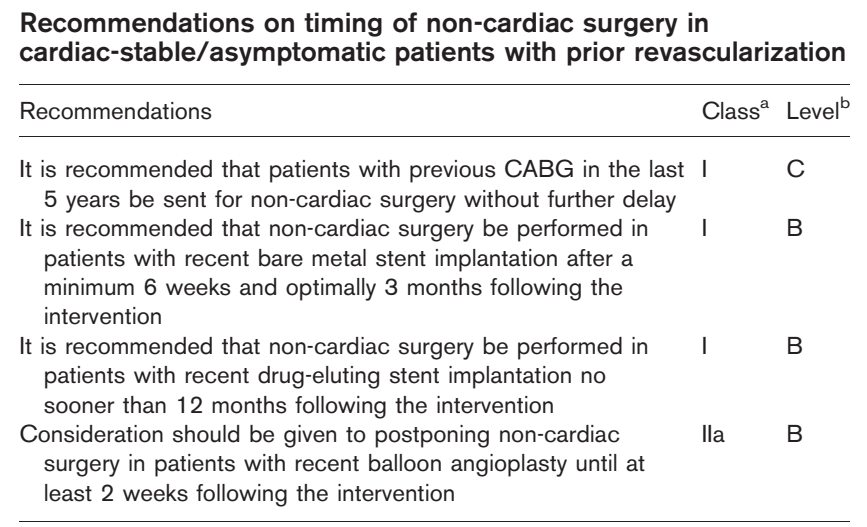

CABG, coronary artery bypass grafting. ${ }^{a}$ Class of recommendation. ${ }^{b}$ Level of evidence.

\section{Prophylactic revascularization in patients with stable ischaemic heart disease}

Only two randomized studies have addressed the role of prophylactic revascularization prior to non-cardiac surgery in stable patients scheduled for vascular surgery. The Coronary Artery Revascularization Prophylaxis (CARP) trial was the first to compare optimal medical therapy with revascularization (by CABG or PCI) in patients with stable IHD prior to major vascular surgery. ${ }^{135}$ Of 5859 patients screened at 18 US Veterans
Affairs hospitals, 510 patients were randomized to one or other of the treatment options. Patients were included on the basis of a combination of cardiovascular risk factors and the detection of ischaemia on non-invasive testing as assessed by the consultant cardiologist. There was no difference in the primary endpoint of long-term mortality at 2.7 years after randomization: $22 \%$ (revascularization) vs. 23\% (no-intervention) $(P=0.92)$. Furthermore, there was no difference in perioperative MI: $12 \%$ vs. $14 \%$, respectively $(P=0.37)$. The second trial, DECREASE$\mathrm{V}$, was a pilot study and applied a different, more precise screening methodology and a more contemporary perioperative medical management. ${ }^{136}$ A total of 1880 patients scheduled for surgery were screened for the presence of the following risk factors: age $>70$ years, angina pectoris, prior MI, compensated or a history of congestive heart failure, drug therapy for diabetes mellitus, renal dysfunction, and prior stroke or transient ischaemic attack (TIA). In the presence of $\geq 3$ risk factors, DSE or nuclear stress testing was performed and in the presence of extensive ischaemia ( $>5 / 16$ segments or $>3 / 6$ walls), patients were randomized to either revascularization or no revascularization. Importantly, $\beta$-blocker therapy was initiated and aspirin was continued during surgery in all patients. Threevessel or left main disease was present in $75 \%$ of cases. Also $43 \%$ of patients had a depressed ejection fraction of $\leq 35 \%$. PCI was performed in $65 \%$ of patients $(n=32$, of whom 30 had DESs). There was no difference in the composite primary endpoint (all-cause mortality and non-fatal MI at 30 days): $43 \%$ for revascularization vs. $33 \%$ for no revascularization $(P=0.30)$.

CARP was the first trial to indicate that prophylactic revascularization prior to vascular surgery does not improve clinical outcomes in stable patients. Nevertheless, inclusion in the trial was based on subjective indicators and the study population was a relatively low risk group. DECREASE-V included high-risk patients with extensive stress-induced ischaemia, as assessed by non-invasive stress testing. Despite the relatively small study cohort, DECREASE-V extends the conclusions of CARP to a higher risk population, with a majority of patients having three-vessel disease and a substantial proportion having asymptomatic LV dysfunction.

Successful achievement of a vascular procedure without prophylactic revascularization in a stable coronary patient does not imply that this patient would not need any revascularization afterwards. The limited data from DECREASE-V indicate a potential late catch-up phenomenon in the medically treated group. ${ }^{136}$ Despite the lack of more scientific data, myocardial revascularization may therefore be recommended in patients prior to foreseen non-cardiac surgery without complications and who present with or have persistent signs of extensive ischaemia, according to the ESC Guidelines for nonsurgical settings. 
Both CARP and DECREASE-V have been conducted in the setting of vascular surgery, a type of surgery presenting particular risk to the patient with coronary heart disease. Despite this limitation, the conclusions of these trials can probably be extrapolated to other types of surgery.

Recommendation for prophylactic revascularization in stable/ asymptomatic patients

\begin{tabular}{lll}
\hline Recommendation & Class $^{\mathrm{a}}$ & Level $^{\mathrm{b}}$ \\
\hline $\begin{array}{l}\text { Late revascularization after successful non-cardiac surgery } \\
\text { should be considered in accordance with }\end{array}$ & $\mathrm{Ila}$ & $\mathrm{C}$ \\
$\begin{array}{l}\text { ESC Guidelines on stable angina pectoris } \\
\text { Prophylactic myocardial revascularization prior to } \\
\text { high-risk surgery may be considered in patients } \\
\text { with proven IHD }\end{array}$ & Illb & B \\
$\begin{array}{l}\text { Prophylactic myocardial revascularization prior to } \\
\text { intermediate-risk surgery in patients with proven IHD } \\
\text { is not recommended }\end{array}$ & III & B \\
$\begin{array}{l}\text { Prophylactic myocardial revascularization prior to } \\
\text { low-risk surgery patients with proven IHD is not } \\
\text { recommended }\end{array}$ & III & C \\
\hline
\end{tabular}

IHD, ischaemic heart disease. ${ }^{a}$ Class of recommendation. ${ }^{b}$ Level of evidence.

Type of prophylactic revascularization in patients with stable ischaemic heart disease

Occasionally, patients with stable IHD may require elective surgery, meaning that surgery may be postponed for several months or even up to $\geq 1$ year. There are no solid data to guide a revascularization strategy in this case, and recommendations can therefore be based only on experts' recommendations. Yet, these patients may to some extent be compared with patients who had previous revascularization. It seems therefore reasonable to propose a cardiovascular work-up according to the ESC Guidelines on stable angina pectoris. ${ }^{47} \mathrm{CABG}$ should be performed to improve prognosis and relieve symptoms in patients with significant left main disease or its equivalent, for significant three-vessel disease, in particular in the case of depressed $\mathrm{LV}$ function, as stated in these guidelines. PCI should be performed to improve symptoms in stable symptomatic patients with single or multivessel disease in whom intervention is technically suitable and in whom the procedural risk does not outweigh the potential benefit. ${ }^{70}$

The choice between PCI and CABG, often a matter of debate, will depend on several factors. Recently, the 1 year results of the Synergy between percutaneous coronary intervention with Taxus and cardiac surgery (SYNTAX) trial, in which 1800 patients with three-vessel or left main IHD were randomized to undergo CABG or PCI, have been published. ${ }^{137}$ They indicate that CABG remains the treatment of choice in these patients but that PCI is a valuable alternative. As mentioned before, current guidelines on the management of stable angina indicate a role for both treatments. Nevertheless, if PCI is performed prior to non-cardiac surgery the use of bare metal stents, in order not to delay surgery unnecessarily, is recommended.
Recommendation on type of prophylactic revascularization in stable patients

\begin{tabular}{lll}
\hline Recommendation & Class $^{\mathrm{a}}$ & Level $^{\mathrm{b}}$ \\
\hline $\begin{array}{l}\text { It is recommended that PCl or CABG be performed } \\
\text { according to the applicable guidelines for management } \\
\text { in stable angina pectoris }\end{array}$ & $\mathrm{I}$ & $\mathrm{A}$ \\
\hline
\end{tabular}

CABG, coronary artery bypass grafting; $\mathrm{PCl}$, percutaneous coronary intervention. ${ }^{\mathrm{a}}$ Class of recommendation. ${ }^{\mathrm{b}}$ Level of evidence.

\section{Revascularization in patients with unstable ischaemic heart disease}

No trial has investigated the role of prophylactic revascularization in patients with unstable angina pectoris requiring non-cardiac surgery. Unstable angina pectoris, in particular non-ST-segment elevation ACS, is considered to be a high-risk clinical entity and requires prompt diagnosis, risk stratification, and revascularization. Therefore, as long as the clinical condition for non-cardiac surgery is not life threatening, priority should be given to the diagnosis and proper treatment of unstable angina. In this case, the recent ESC Guidelines on the management of non-ST-segment elevation ACS apply. ${ }^{69}$ The cornerstone of treatment includes antiplatelet and anticoagulant therapy, $\beta$-blocking agents, and prompt revascularization. Careful attention should be paid to avoiding overt anticoagulation and/or antithrombotic management of unstable coronary patients with concomitant surgical conditions, due to the risk of increased bleeding tendency secondary to the background surgical disease (malignancy, etc.). Except for the previously mentioned well-recognized indications for emergency CABG, most patients undergo PCI. In the exceptional situation of unstable angina and the need for subsequent non-cardiac surgery, preference should again be given to bare metal stents, in order not to delay surgery beyond 3 months.

Recommendations on prophylactic myocardial revascularization in patients with unstable IHD

\begin{tabular}{|c|c|c|}
\hline Recommendations & Class $^{a}$ & Level $^{b}$ \\
\hline $\begin{array}{l}\text { If non-cardiac surgery can be postponed safely, it is } \\
\text { recommended that patients be diagnosed and treated } \\
\text { in line with the guidelines on unstable angina management }\end{array}$ & I & A \\
\hline $\begin{array}{l}\text { In the unlikely combination of a life-threatening clinical } \\
\text { condition requiring urgent non-cardiac surgery and ACS, } \\
\text { it is recommended that surgery be given priority }\end{array}$ & 1 & C \\
\hline $\begin{array}{l}\text { However, on follow-up, aggressive medical treatment and } \\
\text { myocardial revascularization according to the guidelines } \\
\text { on unstable angina pectoris management is recommended }\end{array}$ & I & B \\
\hline $\begin{array}{l}\text { If } \mathrm{PCl} \text { is indicated, the use of bare metal stents or even } \\
\text { balloon angioplasty is recommended }\end{array}$ & 1 & C \\
\hline
\end{tabular}

$\mathrm{ACS}$, acute coronary syndrome; $\mathrm{HD}$, ischaemic heart disease; $\mathrm{PCl}$, percutaneous coronary intervention. ${ }^{\mathrm{a}}$ Class of recommendation. ${ }^{\mathrm{b}}$ Level of evidence.

\section{Specific diseases}

So far, the guidelines have discussed cardiac risk markers and risk reduction strategies. However, patients 
presenting with specific diseases prior to surgery benefit from an integrated evaluation and management of their disease in the perioperative period. In the following sections the most common cardiovascular diseases are discussed.

\section{Chronic heart failure}

The prevalence of chronic heart failure in the adult population in the UK has been estimated to be $1.8 \%$, and this increases with age. In patients $>75$ years the prevalence is as high as $8.0 \%$.

The predictive value of heart failure for perioperative cardiac events is well recognized and is an important factor of clinical risk indices, such as Goldman's or Detsky's risk score ${ }^{31,32}$ A study evaluating $L V$ function prior to vascular surgery in 1988 found an LV ejection fraction of $\leq 35 \%$ to be an optimal predictor of post-operative cardiac events. ${ }^{138}$ In 2008, another study confirmed these findings and concluded that elderly patients with chronic heart failure scheduled for vascular surgery have higher risks of operative mortality and hospital readmission than other patients (including those with IHD) admitted for the same procedure. ${ }^{139}$ The prognostic pre-operative value of heart failure with preserved LV ejection fraction is ill defined. Long-term outcome is similar to that of patients with reduced LV ejection fraction. ${ }^{140}$ These patients could present an increased cardiovascular risk when undergoing surgery. In the absence of evidence-based studies, the committee recommends similar perioperative management in patients with preserved $L V$ ejection fraction as in patients with a reduced ejection fraction.

The ability to assess myocardial viability during stress testing has allowed further risk stratification of cases with LV dysfunction. As shown in a study of 295 patients with a $\mathrm{LV}$ ejection fraction $<35 \%$ scheduled for vascular surgery, post-operative cardiac events were related to the presence of stress-induced ischaemia and scar tissue. ${ }^{141}$ However, there was an inverse relationship to the presence and extent of dysfunctional but viable segments, showing an improved function without signs of ischaemia during inotropic stimulation. Using multivariable analysis, the number of ischaemic segments was associated with perioperative cardiac events (OR per segment 1.6, 95\% CI 1.05-1.8), whereas the number of segments with sustained improvement was associated with improved outcome (OR per segment $0.2,95 \%$ CI 0.04-0.7). The stratification using stress testing enables the physician to identify a subgroup of patients with sustained improvement who have a relatively benign post-operative outcome, unlike patients with a predominantly ischaemic response.

Current ESC Guidelines recommend the use of ACE inhibitors (or ARBs in patients intolerant of ACE inhibitors) and $\beta$-blockers as primary treatment in chronic heart failure patients, to improve morbidity and mortality. ${ }^{91}$ Unless contra-indicated or not tolerated, they should be given in optimal doses in all patients with symptomatic heart failure and an LV ejection fraction $\leq 40 \%$. Either an $\mathrm{ARB}$ or an aldosterone antagonist may subsequently be added, depending on clinical condition and patient characteristics. In all patients with an $L V$ ejection fraction $\leq 35 \%$ who remain severely symptomatic [New York Heart Association (NYHA) functional class III or IV], the addition of a low dose of aldosterone antagonist should be considered (in the absence of hyperkalaemia and significant renal dysfunction). As an alternative option, addition of an ARB is recommended in heart failure patients with an $\mathrm{LV}$ ejection fraction $\leq 40 \%$ who remain symptomatic despite optimal treatment with an ACE inhibitor and $\beta$-blocker, unless also taking an aldosterone antagonist. Diuretics are recommended in heart failure patients with signs or symptoms of congestion.

It has been concluded that the perioperative use of ACE inhibitors, $\beta$-blockers, statins, and aspirin is independently associated with a reduced incidence of in-hospital mortality in patients with $\mathrm{LV}$ dysfunction who are undergoing major non-cardiac vascular surgery. ${ }^{142}$ Thus, it is recommended that life-saving therapies in stable heart failure patients be continued up until the surgery and that they be reinstituted post-operatively, as soon as clinical conditions are satisfactory.

The diagnosis of post-operative heart failure is often difficult to make since it often presents atypically and may have a different aetiology from that in the nonsurgical setting. The evaluation should include physical examination, ECG, serial biomarker measurements, Xray, and echocardiography. Special attention should be given to the patient's volume status since high-volume infusion is often needed in the intra- and immediate postoperative setting. In the period after surgery, fluids given during the operation may be mobilized to cause hypervolaemia and even heart failure, if not adequately handled. Fluid overloading may cause decompensation of chronic heart failure or development of de novo acute heart failure. Heart failure may develop perioperatively either immediately after surgery (due to prolonged procedure, myocardial ischaemia, rapid fluid shift) or some days later (due to third-space fluid re-absorption). According to the recent ESC Guidelines on heart failure, an attempt should be made to optimize pharmacological therapy before surgery. This may be of particular importance for $\beta$-blockers, which are recommended in the perioperative period in all high-risk patients. To avoid uncontrolled hypotension, routine use of i.v. $\beta$-blockers is not recommended. Importantly, if a heart failure patient is not receiving a $\beta$-blocker, such therapy should be initiated early enough before elective surgery to ensure optimal dose uptitration. 
Once the aetiology of post-operative heart failure is diagnosed, treatment is similar to the non-surgical setting. Patients with heart failure have a significantly higher risk of hospital readmission after surgical procedures. This confirms the need for careful discharge planning and close follow-up, optimally using a multidisciplinary approach.

\section{Arterial hypertension}

In general, the presence of arterial hypertension is not considered to be an independent risk factor for cardiovascular complications in non-cardiac surgery. Preoperative evaluation allows the identification of patients with hypertension, enables a search for target organ damage and evidence of associated cardiovascular pathology to be undertaken, and allows initiation of appropriate therapy. This is particularly important for those with concomitant risk factors.

There is no clear evidence favouring one mode of antihypertensive therapy over another in patients undergoing non-cardiac surgery. Patients with arterial hypertension should be managed according to existing ESC Guidelines. ${ }^{143}$ However, in hypertensive patients with concomitant IHD who are at high risk of cardiovascular complications, perioperative administration of $\beta$-blockers is recommended. In patients with hypertension, antihypertensive therapy should be continued up to the morning of surgery and restarted promptly in the post-operative period. ${ }^{144}$ In patients with grade 1 or 2 hypertension, ${ }^{143}$ there is no evidence that delay in surgery in order to optimize therapy is beneficial. In these cases, antihypertensive medications should be continued during the perioperative period. In patients with grade 3 hypertension (systolic blood pressure $180 \mathrm{mmHg}$ and/or diastolic blood pressure $110 \mathrm{mmHg}$ ), the potential benefits of delaying surgery to optimize the pharmacological therapy should be weighed against the risk of delaying the surgical procedure. ${ }^{20,144}$

\section{Valvular heart disease}

Patients with VHD are at higher risk of perioperative cardiovascular complications during non-cardiac surgery. ${ }^{124}$ Echocardiography should be performed in patients with known or suspected VHD, to assess its severity and consequences. On the basis of existing data, the following recommendations are particularly applicable in these patients. ${ }^{20,124}$

\section{Recommendation on VHD}

\begin{tabular}{lll}
\hline Recommendation & Class $^{\mathrm{a}}$ & Level $^{\mathrm{b}}$ \\
\hline In the presence of severe VHD it is recommended that a & $\mathrm{C}$ \\
clinical and echocardiographic evaluation be performed \\
and, if needed, treatment before non-cardiac surgery \\
\hline VHD, valvular heart disease. ${ }^{\mathrm{a}}$ Class of recommendation. ${ }^{\mathrm{b}}{ }^{\text {Level of evidence. }}$
\end{tabular}

\section{Aortic stenosis}

Aortic stenosis (AS) is the most common VHD in Europe, particularly among the elderly. ${ }^{145}$ Severe AS (defined as aortic valve area $<1 \mathrm{~cm}^{2},<0.6 \mathrm{~cm}^{2} / \mathrm{m}^{2}$ body surface area) constitutes a well-established risk factor for perioperative mortality and MI. ${ }^{146}$ In the case of urgent non-cardiac surgery in patients with severe AS, such procedures should be performed under haemodynamic monitoring. ${ }^{124}$ In the case of elective non-cardiac surgery, the presence of symptoms is a key for decision making.

In symptomatic patients, aortic valve replacement should be considered before elective surgery. In patients who are not candidates for valve replacement due to either high risks associated with serious co-morbidities or those who refuse, non-cardiac surgery should be performed only if is essential. In these patients, balloon aortic valvuloplasty or transcatheter valve implantation may be a reasonable therapeutic option before surgery. ${ }^{124,147}$

In asymptomatic patients, non-cardiac surgery of low to intermediate risk can be safely performed. If high-risk surgery is planned, further clinical assessment is necessary for aortic valve replacement. In those at high risk for aortic valve replacement, elective surgery under strict haemodynamic monitoring should be performed only if strictly needed. In the remaining patients, aortic valve replacement should be considered as the initial procedure. $^{124}$

\section{Mitral stenosis}

Non-cardiac surgery can be performed at relatively low risk in patients with non-significant mitral stenosis (MS) (valve area $>1.5 \mathrm{~cm}^{2}$ ) and in asymptomatic patients with significant MS (valve area $<1.5 \mathrm{~cm}^{2}$ ) and systolic pulmonary artery pressure $<50 \mathrm{mmHg}$. Pre-operative surgical correction of MS in these patients is not indicated. It needs to be remembered that control of heart rate is essential to avoid tachycardia, which may cause pulmonary oedema. Strict control of fluid overload is also important. Also development of AF may cause serious clinical deterioration. ${ }^{20,124}$ With the high risk of embolism, anticoagulation control is important. In asymptomatic patients with significant MS and systolic pulmonary artery pressure $>50 \mathrm{mmHg}$ and in symptomatic patients, the risk related to the non-cardiac procedure is significantly higher, and these patients may benefit from percutaneous mitral commissurotomy (or open surgical repair) particularly before high-risk surgery. ${ }^{20,124}$

\section{Aortic regurgitation and mitral regurgitation}

Non-significant aortic regurgitation (AR) and mitral regurgitation (MR) do not independently increase the risk of cardiovascular complications during non-cardiac surgery. In asymptomatic patients with severe AR and MR (detailed classification presented in the ESC Guidelines ${ }^{124}$ ) and preserved LV function, non-cardiac surgery 
can be performed without additional risk. Symptomatic patients and those who are asymptomatic with severely impaired $\mathrm{LV}$ ejection fraction $(<30 \%)$ are at high risk of cardiovascular complications, and non-cardiac surgery should be performed only if necessary. ${ }^{124}$ Patients with severe MR and AR may benefit from optimization of pharmacological therapy to produce maximal haemodynamic stabilization before high-risk surgery.

\section{Patients with prosthetic valve(s)}

Patients who have undergone surgical correction of VHD and have a prosthetic valve can undergo non-cardiac surgery without additional risk, when there is no evidence of valve or ventricular dysfunction. In these patients, endocarditis prophylaxis is recommended and a modification of the anticoagulation regimen needs to be considered in the perioperative period, with oral anticoagulants being temporarily replaced by i.v. UFH, s.c. UFH, or s.c. LMWH at therapeutic doses.

\section{Prophylaxis of infective endocarditis}

In patients with VHD and those with prosthetic valves who are undergoing non-cardiac surgery at risk of bacteraemia, antibiotic prophylaxis against infective endocarditis should be initiated. This issue is discussed in detail in the ESC and AHA guidelines. ${ }^{148,149}$

\section{Arrhythmias}

The occurrence of perioperative arrhythmias has been reported in $70 \%$ of patients subjected to general anaesthesia for various surgical procedures. ${ }^{150,151}$ The incidence has been reported to vary from 16 to $62 \%$ with intermittent ECG monitoring ${ }^{152}$ and $89 \%$ with continuous Holter monitoring. ${ }^{153}$

\section{Ventricular arrhythmias}

Almost half of all high-risk patients undergoing noncardiac surgery have frequent ventricular premature beats (VPBs) or non-sustained VT. There is no evidence that VPBs or non-sustained VTs alone are associated with a worse prognosis. ACC/AHA/ESC Guidelines for management of patients with ventricular arrhythmias and the prevention of sudden cardiac death recommend approaches based on large clinical trials. ${ }^{154}$ Regardless of the cause, sustained monomorphic ventricular tachycardia (SMVT) with serious haemodynamic compromise must be treated promptly with electric cardioversion. ${ }^{154}$ Intravenous amiodarone can be used for initial treatment of patients with stable SMVT. ${ }^{154}$ It is also reasonable in patients with SMVT that is haemodynamically unstable, refractory to conversion with countershock, or recurrent despite other agents. In sustained polymorphic ventricular tachycardia (SPV'T), if haemodynamic compromise is present, immediate electrical cardioversion should be performed. $\beta$-Blockers are useful for patients with recurrent SPVT, especially if ischaemia is suspected or cannot be excluded. Amiodarone is reasonable for patients with recurrent SPVT in the absence of long QT syndrome (LQTS). ${ }^{154}$ Torsades de pointes rarely occurs, and withdrawal of any offending drugs and correction of electrolyte abnormalities are recommended. Management with magnesium sulfate is reasonable for patients with torsades de pointes and LQTS. $\beta$-Blockade combined with pacing is suggested in patients who have torsades de pointes and sinus bradycardia. Isoproterenol is recommended in patients with recurrent pause-dependent torsades de pointes who do not have congenital LQTS. ${ }^{154}$ In the event of perioperative pulseless VT or ventricular fibrillation, immediate defibrillation is required.

\section{Supraventricular arrhythmias}

A greater number of patients undergoing non-cardiac surgery may suffer from SVT and AF compared with ventricular arrhythmias. ${ }^{153-158}$ Sympathetic activity is the primary autonomic mechanism responsible for the trigger of AF. ${ }^{159}$ Vagal manoeuvres may terminate SVT in some cases and these arrhythmias respond well to treatment with adenosine. When SVT is refractory to adenosine, effective therapy for termination of the arrhythmia includes a short-acting $\beta$-blocking agent or a nondihydropyridine calcium channel blocker (diltiazem and verapamil) or amiodarone i.v. ${ }^{160-162}$ Verapamil should be used with care because of its negative inotropic effect. The use of calcium channel blockers is not recommended in pre-excited SVT/AF. For perioperative AF, the goal of management is ventricular rate control. ${ }^{163} \beta$-Blockers and non-dihydropyridine calcium channel blockers (diltiazem and verapamil) are the drugs of choice for the rate control in AF. Digoxin may be used as a first-line drug only in patients with chronic heart failure, since it is not effective in high adrenergic states such as surgery. $\beta$-Blockers have been shown to accelerate the conversion of AF to sinus rhythm after non-cardiac surgery. ${ }^{164}$ In several studies, the pre-operative administration of $\beta$-blockers was associated with better control of arrhythmias. ${ }^{165,166}$

\section{Bradyarrhythmias}

Severe perioperative bradyarrhythmias requiring treatment have been reported in $0.4 \%$ of 17021 patients, $6.4 \%$ of whom were American Association of Anesthesiologists physical status 3 or $4 .{ }^{151}$ These patients were monitored with routine intraoperative and early post-operative ECG monitoring. In general, perioperative bradyarrhythmias respond well to short-term pharmacological therapy, noninvasive transoesophageal atrial pacing in anaesthetized individuals, or non-invasive transcutaneous pacing in awake or anaesthetized patients. ${ }^{160}$ Temporary cardiac pacing is rarely required, even in the presence of preoperative asymptomatic bifascicular block or left bundle branch block. ${ }^{167}$ The indications for temporary pacemakers during the perioperative period are generally the same as those for permanent pacemakers. ${ }^{168}$ Asymptomatic bifascicular block, with or without first degree 
atrio-ventricular block, is not an indication for temporary endocardial pacing. ${ }^{169,170}$

\section{Pacemaker/implantable cardioverter defibrillator}

The use of unipolar electrocautery represents a significant risk to pacemaker-dependent patients. The electrical stimulus from electrocautery may inhibit demand pacemakers or may reprogramme the pacemaker. However, these problems can be avoided by positioning the ground plate for the electrical circuit, such that the electrical current travels away from the generator. Keeping the electrocautery device away from the pacemaker, giving only brief bursts and using the lowest possible amplitude may decrease the interference. In many studies, the authors recommended setting the pacemaker in an asynchronous or non-sensing mode in patients who are pacemaker dependent and whose underlying rhythm is unreliable, and interrogating the device after surgery to ensure appropriate programming and sensing pacing thresholds. ${ }^{171-174}$ Interference with implantable cardioverter defibrillator function can also occur during noncardiac surgery as a result of electrical current generated by electrocautery. ${ }^{175,176}$ The implantable cardioverter defibrillator should be turned off during surgery and switched on in the recovery phase before discharge to the ward. In addition, it is recommended that written instructions regarding the responsibility for surveillance and restarting of the implantable cardioverter defibrillator should be available.

\section{Renal disease}

Reduced kidney function is an independent risk factor for adverse post-operative cardiovascular outcomes including MI, stroke, and progression of heart failure. In most risk indices, renal function is taken into account. Traditionally, this function is assessed by serum creatinine concentration. For example, the serum creatinine cut-off value of $>2.0 \mathrm{mg} / \mathrm{dL}$ $(177 \mu \mathrm{mol} / \mathrm{L})$ is used in the Lee index. ${ }^{5}$ However, estimated creatinine clearance $(\mathrm{mL} / \mathrm{min})$ incorporating serum creatinine, age, and weight provides a more accurate assessment of renal function than serum creatinine alone. Most commonly used is the CockcroftGault formula $\{[(140$ - age in years) (weight in $\mathrm{kg})] /[72$ serum creatinine in $\mathrm{mg} / \mathrm{dL}]\}$ (0.85 for females). ${ }^{177} \mathrm{An}$ evaluation of 852 subjects undergoing major vascular surgery demonstrated an increase in mortality when serum creatinine was $>2.0 \mathrm{mg} / \mathrm{dL}$ with an $\mathrm{OR}$ for perioperative mortality of 5.2 , 95\% CI 2.9-10.8. ${ }^{178}$ However, it might be argued that patients with less pronounced renal insufficiency also do worse than patients with normal serum creatinine values. A $10 \mathrm{~mL} / \mathrm{min}$ decrease in creatinine clearance was associated with a $40 \%$ increased risk of post-operative mortality (OR 1.4, 95\% CI 1.2-1.5; ROC area: 0.70, 95\% CI 0.63-0.76). ROC curve analysis showed that the cut-off value of $64 \mathrm{~mL} / \mathrm{min}$ for creatinine clearance yielded the highest sensitivity/specificity to predict post-operative mortality. ${ }^{178}$

\section{Recommendations on ventricular arrhythmias}

\begin{tabular}{|c|c|c|}
\hline Recommendations & Class $^{a}$ & Level $^{\mathrm{b}}$ \\
\hline Anti-arrhythmic drugs are recommended for patients with recurrent sustained VT & I & $\mathrm{B}$ \\
\hline Continuation of amiodarone and $\beta$-blockers before surgery is recommended & I & $\mathrm{C}$ \\
\hline It is recommended that wide QRS tachycardia be considered to be VT if the diagnosis is unclear & I & C \\
\hline Prompt electrical cardioversion in patients with sustained VT with haemodynamic compromise is recommended & I & $\mathrm{C}$ \\
\hline Anti-arrhythmic drugs for initial treatment of patients with stable sustained monomorphic VT should be considered & Ila & $\mathrm{B}$ \\
\hline Anti-arrhythmic drugs for patients with non-sustained VT are not recommended & III & $\mathrm{B}$ \\
\hline Anti-arrhythmic drugs for patients with VPBs are not recommended & III & A \\
\hline
\end{tabular}

VPB, ventricular premature beat; VT, ventricular tachycardia. ${ }^{a}$ Class of recommendation. ${ }^{\text {b }}$ Level of evidence.

Recommendations on supraventricular arrhythmias

\begin{tabular}{ll} 
Recommendations & Class $^{\text {a }}$ \\
\hline Ventricular rate control is recommended in patients with AF without haemodynamic instability & Level $^{\text {b }}$ \\
Continuation of oral anti-arrhythmic drugs before surgery is recommended & I \\
Vagal manoeuvres and anti-arrhythmic therapy for termination of SVT in haemodynamic stable patients is recommended & $\mathrm{C}$ \\
\hline
\end{tabular}

AF, atrial fibrillation; SVT, supraventricular tachycardia. ${ }^{a}$ Class of recommendation. ${ }^{b}$ Level of evidence.

Recommendations on implantable devices

\begin{tabular}{|c|c|c|}
\hline Recommendations & Class $^{a}$ & Level $^{\mathrm{b}}$ \\
\hline Interrogation of implantable devices pre-operatively and post-operatively is recommended & I & $\mathrm{C}$ \\
\hline It is recommended that the hospital management state who is responsible for programming the devices before and after surgery & I & C \\
\hline
\end{tabular}

${ }^{\mathrm{a}}$ Class of recommendation. ${ }^{\mathrm{b}}$ Level of evidence. 
In addition to the pre-operative renal function, worsening of function after surgery is a prognostic factor for adverse late outcome. In 1324 patients who underwent elective open AAA surgery, creatinine clearance was measured pre-operatively and on days 1,2 , and 3 after surgery. ${ }^{179}$ Patients were divided into three groups according to the change in renal function after surgery compared with baseline. Group 1 showed an improved or no change (change in creatinine clearance, $\pm 10 \%$ of function compared with baseline); group 2 showed a temporary worsening (worsening $>10 \%$ at day 1 or 2 , then complete recovery within $10 \%$ of baseline at day 3 ); and group 3 experienced a persistent worsening $(>10 \%$ decrease compared with baseline). Mortality during 30 days after surgery was $1.3,5.0$, and $12.6 \%$ in groups 1,2 , and 3 , respectively. Adjusted for baseline characteristics and post-operative complications, 30-day mortality was highest in patients with persistent worsening of renal function (HR 7.3, 95\% CI 2.7-19.8), followed by those with temporary worsening (HR 3.7, 95\% CI 1.4-9.9). During $6.0 \pm 3.4$ years of follow-up, 348 patients $(36.5 \%)$ died. The risk of late mortality was 1.7 (95\% CI $1.3-2.3)$ in the persistent worsening group followed by those with temporary worsening (HR 1.5, 95\% CI 1.2-1.4). This study showed that, although renal function may recover completely after aortic surgery, temporary worsening of renal function was associated with an increased long-term mortality. ${ }^{179}$

Identification of patients who might experience perioperative worsening of renal function is important in order to initiate supportive measures such as maintenance of adequate intravascular volume for renal perfusion and vasopressor use. In a large retrospective study, risk factors for post-operative acute renal failure within the first 7 days after major non-cardiac surgery among patients with previously normal renal function were evaluated. ${ }^{180}$ Thirty-day, 60-day, and 1-year all-cause mortality was also assessed. A total of 65043 cases throughout 2003 and 2006 were reviewed. Of these, 15102 patients met the inclusion criteria; 121 patients developed acute renal failure $(0.8 \%)$, and 14 required renal replacement therapy $(0.1 \%)$. Seven independent pre-operative predictors were identified $(P<0.05)$ : age, emergency surgery, liver disease, high body mass index, high-risk surgery, peripheral arterial occlusive disease, and COPD necessitating chronic bronchodilator therapy.

Contrast-induced nephropathy, caused by renal hypoperfusion and direct tubular toxicity, occurs in up to $15 \%$ of patients with chronic renal dysfunction undergoing radiographic procedures. ${ }^{181}$ Between 0.5 and $12 \%$ of these patients require haemodialysis and prolonged hospitalization. A considerable number of patients experience worsening of renal function, possibly progressing to end-stage renal failure. The cornerstone of prevention consists of periprocedural hydration and antioxidant drugs. Recently, three randomized studies have compared the effects of sodium bicarbonate vs. isotonic saline in humans, resulting in an impressive reduction in contrast nephropathy in the sodium bicarbonate group, with an incidence $<2 \% .{ }^{182}$ These results were recently evaluated in an adequately powered randomized trial comparing the efficacy of hydration with sodium bicarbonate vs. isotonic saline in addition to oral $N$-acetylcysteine for prophylaxis of contrast-induced nephropathy in a population of patients with chronic kidney dysfunction undergoing planned coronary angiography or intervention. A total of 502 patients with an estimated creatinine clearance $<60 \mathrm{~mL} / \mathrm{min}$ were randomized to receive infusion of either saline $(0.9 \% \mathrm{NaCl})$ or sodium bicarbonate before and after administration of contrast medium on top of $\mathrm{N}$ acetylcysteïne orally $\left(600 \mathrm{mg}\right.$ b.i.d.). ${ }^{183}$ Treatment with isotonic saline consisted of $1 \mathrm{~mL} / \mathrm{kg} / \mathrm{h} \quad 0.9 \%$ sodium chloride for $12 \mathrm{~h}$ before and after the procedure, and treatment with sodium bicarbonate $(154 \mathrm{mEq} / \mathrm{L}$ in dextrose and water) consisted of $3 \mathrm{~mL} / \mathrm{kg}$ for $1 \mathrm{~h}$ before the contrast medium, followed by an infusion of $1 \mathrm{~mL} / \mathrm{kg} / \mathrm{h}$ for $6 \mathrm{~h}$ after the procedure. Contrast-induced nephropathy was defined as an absolute increase in serum creatinine $\geq 0.5 \mathrm{mg} / \mathrm{dL}$ measured within 5 days after contrast exposure. No difference was observed between the two study groups; contrast-induced nephropathy occurred in 54 patients $(10.8 \%) ; 25(10 \%)$ were treated with sodium bicarbonate and $29(11.5 \%)$ with saline $(P=0.60)$. Thus, hydration with sodium bicarbonate plus oral $N$-acetylcysteine before contrast medium exposure was no more effective than hydration with isotonic sodium chloride plus oral $\mathrm{N}$-acetylcysteine for prophylaxis of contrastinduced nephropathy in patients with moderate renal dysfunction. The discrepancies among randomized studies might be explained by differences in the concomitant use of $N$-acetylcysteine, use of contrast medium, or baseline renal dysfunction among randomized patients. Sodium bicarbonate requires only $1 \mathrm{~h}$ of pretreatment and may represent an option in patients scheduled for urgent agent injection or for outpatient procedures.

\begin{tabular}{|c|c|c|}
\hline Recommendation/statement & Class $^{a}$ & Level $^{b}$ \\
\hline $\begin{array}{l}\text { It is recommended that pre-operative renal function be } \\
\text { considered as an independent cardiac risk factor for } \\
\text { perioperative and long-term prognosis }\end{array}$ & I & $\mathrm{B}$ \\
\hline $\begin{array}{l}\text { For patients at risk of developing contrast-induced } \\
\text { nephropathy }(\mathrm{CIN}) \text {, hydration with isotonic sodium chloride } \\
\text { (with or without oral } N \text {-acetylcysteine) for prophylaxis of } \\
\mathrm{CIN} \text { is recommended prior to cardiac imaging procedures } \\
\text { that are involved with administration of contrast medium } \\
\text { injection (e.g. coronary and/ or peripheral angiography) }\end{array}$ & I & B \\
\hline
\end{tabular}

${ }^{a}$ Class of recommendation. ${ }^{b}$ Level of evidence.

\section{Cerebrovascular disease}

Cerebrovascular disease is the third leading cause of death in Western countries, with 500 TIAs and 2400 
new strokes per million inhabitants. One-third of new stroke patients die within 1 year, and $<50 \%$ make a full recovery and regain independence. An increasing number of elderly patients are referred for non-cardiac surgery, including those with concomitant vascular diseases affecting the cerebral circulation. Risk factors for perioperative symptomatic or asymptomatic transient or permanent cerebrovascular events (TIA/stroke) are embolism or haemodynamic compromise in large (aorta, carotid, vertebral, and main cerebral arteries intracranially) or small vessels (perforating and penetrating arterioles and capillaries). Although fatal and nonfatal stroke can be reduced significantly in symptomatic patients with moderate/severe carotid stenosis associated with ipsilateral symptoms, in particular if treated early (2-4 weeks, but at least within 3-6 months after the onset of symptoms), the benefit of this interventional/surgical treatment is smaller in neurologically asymptomatic subjects. Thus medical measures to prevent stroke are of utmost general importance and include a multifaceted strategy aimed at control of hypertension, hyperlipidaemia, diabetes, etc. The usefulness of specific antiplatelet agents or anticoagulants has been demonstrated in many randomized controlled trials for primary and secondary prevention, and may even be increased in elderly subjects undergoing non-cardiac surgery and anaesthesia. ${ }^{184}$

Apart from stroke and TIA, transient or permanent changes in mental status characterized by disturbances of attention, orientation, memory dysfunction, illusions, hallucinations, aphasia, etc. (the key diagnostic features of delirium) may occur, including anxiety and depression, which are often under-recognized or misdiagnosed. They may be due to perioperative medication, surgery itself, intraoperative hypo- or hypertension, and cerebral microembolism causing multiple small vessel occlusion and ischaemia, evidenced by transcranial Doppler and MRI diffusion-weighted imaging. In cardiac surgery, mental changes are common and may be associated with transient and occasionally even permanent cognitive dysfunction $(25-30 \%)$. It is very likely that they also occur in the elderly high-risk patient undergoing non-cardiac surgery.

Current concepts of perioperative stroke are summarized in three major reviews ${ }^{185-187}$ which compare the incidence of stroke for various surgical procedures $(0.08$ $0.07 \%$ in general surgery, $1-5 \%$ in peripheral and carotid surgery, and $2-10 \%$ in cardiac surgery). Contrary to common belief, most strokes are not related to hypoperfusion, but occur mainly in the presence of an intact cerebral autoregulation. ${ }^{187}$ Ischaemic and embolic mechanisms are far more common than haemodynamic compromise. Delayed stroke is mainly attributed to various sources of cardiac embolism, followed by hypercoagulability and increased risk of thrombogenic events. Many strokes remain undiagnosed because of a lack of major sensory-motor symptoms or the presence of only subtle neuropsychological deficits, which are more difficult to identify. Several patient- and procedure-related factors are associated with an increased risk of perioperative stroke-they should be investigated carefully to evaluate the individual risk/benefit ratio and optimize care, including appropriate risk modification and timing of surgery. A history of recent stroke or TIA is the strongest predictor for perioperative stroke and should be identified after evaluating the history and the neurological status of each patient. In such cases, and if in doubt, additional brain and vascular images are recommended. In patients with both carotid and cardiac disease, death rates from cardiac causes exceed the risk of stroke; a review of the literature from 1970 to 2000 showed that patients with significant asymptomatic carotid stenosis are at high risk for fatal and non-fatal cardiac events (8\%/year), but not for stroke (1-2\%/year). ${ }^{96}$ However, the overall perioperative stroke risk tends to be overstated. There is no evidence-based recommendation to treat carotid stenosis prior to noncardiac surgery, but there are exceptional cases prior to cardiac surgery.

Discontinuation of warfarin or antiplatelet agents in anticipation of surgery exposes patients to an increased risk of perioperative stroke. A review of perioperative outcome in patients requiring warfarin showed $0.6 \%$ thromboembolic events in those who continued therapy vs. $7.0 \%$ in patients who received i.v. heparin as bridging therapy. ${ }^{188}$ Whether this is due to insufficient control or dosage of heparin administration is uncertain. In knee or hip replacement, continued use of moderate dose warfarin therapy during the perioperative period was safe and effective and was similar to patients undergoing dental procedures, cataract surgery, and diagnostic endoscopy without interrupting their anti-platelet agents or oral anticoagulants regimen. Lengthy operations are associated with higher risks for perioperative stroke; the choice of surgical technique is also important and the types of anaesthesia and anaesthetic agents require additional consideration. Optimal selection of individually guided best levels of blood pressure during surgery and thereafter, as well as management of the patient's body temperature and control of blood glucose, are suggested to reduce rates of incidental stroke and death. Pre-, intra-, and post-operative use of antiplatelet agents is useful. Whether or not so-called neuroprotective agents are needed is a matter of controversy.

Recommendations on stroke/transient ischaemic attack (TIA)

\begin{tabular}{lll}
\hline Recommendations & Class $^{\mathrm{a}}$ & Level $^{\mathrm{b}}$ \\
\hline $\begin{array}{l}\text { If carotid stenosis is }>70 \% \text {, additional therapy such as } \\
\text { antiplatelet therapy and/or surgery is recommended }\end{array}$ & $\mathrm{I}$ & $\mathrm{A}$ \\
$\begin{array}{l}\text { Routine pre-operative screening for symptomatic or } \\
\text { asymptomatic carotid stenosis may be considered }\end{array}$ & $\mathrm{Ilb}$ & $\mathrm{C}$ \\
\hline
\end{tabular}

${ }^{\mathrm{a}}$ Class of recommendation. ${ }^{\mathrm{b}}$ Level of evidence. 


\section{Pulmonary disease}

The co-existence of pulmonary disease in patients having non-cardiac surgery may increase the risk of operation. Such diseases include acute respiratory infections, COPD, asthma, cystic fibrosis, interstitial lung disease, and other conditions causing impairment of respiratory function. Pre-existing pulmonary disease has a significant impact on perioperative risk, but the most common effect is to increase the risk of post-operative pulmonary complications. These complications are mainly a consequence of the development of atelectasis during general anaesthesia. Post-operative shallow breathing, reduced lung expansion, and other factors may cause the lung collapse to persist and promote respiratory infection. These complications occur especially after abdominal or thoracic surgery, and the risk seems to be increased in smokers. Specific perioperative management is required to reduce the risks of pulmonary complications. There are some respiratory conditions which are associated with cardiovascular abnormalities and which may require special cardiac risk assessment and management in addition to dealing with pulmonary complications perse. Two such conditions are COPD and pulmonary arterial hypertension (PAH).

COPD, defined as airways obstruction which is not completely reversible, is well recognized as a major cause of morbidity and mortality. The prevalence of COPD in adults in Europe has been found to vary between $\sim 5$ and $10 \%$, with rates tending to be higher in males than females. Thus, up to one in 10 patients having noncardiac surgery may have COPD.

Cor pulmonale with right heart failure is a direct complication of severe COPD. However, COPD is also associated with an increased risk of coronary heart disease. In a systematic review of 12 population cohort studies, those with a reduced forced expiratory volume in $1 \mathrm{~s}\left(\mathrm{FEV}_{1}\right)$ had a $75 \%$ increased risk of cardiovascular mortality compared with those with a normal $\mathrm{FEV}_{1} \cdot{ }^{189}$ Reduced expiratory flow has also been associated with a higher incidence of non-fatal coronary heart disease and stroke, carotid stenosis, low ankle-brachial index, and cerebral white matter lesions. These associations occur in both men and women and, despite a strong relationship of smoking with both COPD and CVD, are independent of traditional cardiovascular risk factors. For every $10 \%$ decrease in $\mathrm{FEV}_{1}$, cardiovascular mortality increases by $\sim 30 \%$ and non-fatal coronary events by $\sim 20 \%$.

In patients undergoing aortic aneurysm repair, conflicting results have been found with short-term mortality (often due to cardiac complications). For example, COPD has been associated with operative death, but not 30-day mortality. In vascular surgery patients as a whole, COPD has not been associated with increased 30-day mortality. Thus, despite an association with GVD, there is no convincing evidence that COPD is related to a higher risk of perioperative cardiac complications.

PAH may be idiopathic, due to congenital heart disease, familial, or associated with specific conditions such as collagen vascular disease. It must be distinguished from other causes of PAH due to COPD, thromboembolism, and congenital disease. The diagnosis is based on a mean arterial pulmonary pressure of $>25 \mathrm{mmHg}$ at rest and a pulmonary wedge pressure of $\leq 15 \mathrm{mmHg}$. In surveys in Europe, the prevalence has varied between about 15 and 50 cases per million adults. Half the cases were idiopathic. The prevalence is thus low and consequently the condition is uncommon in surgical practice.

PAH increases surgical complications, especially right ventricular failure, myocardial ischaemia, and postoperative hypoxia. In patients having cardiopulmonary bypass surgery, a mean pre-operative arterial pressure $>30 \mathrm{mmHg}$ is an independent predictor of mortality. In a study of patients with pulmonary hypertension undergoing non-cardiac surgery, of whom over half had PAH, outcome predictors included NYHA functional class $\geq \mathrm{II}$, intermediate- to high-risk surgery, right ventricular function, and duration of anaesthesia. ${ }^{190}$ There is a need for further research on factors predicting poor outcomes. However, the study above did confirm that such patients are at high risk, the perioperative cardiopulmonary complication rate being $38 \%$ and mortality $7 \%$.

Pre-existing COPD is often considered in terms of the risk of post-operative pulmonary complications. For perioperative cardiac risk, the lack of convincing evidence that COPD increases risk may have arisen because in COPD patients extra care was taken with cardiac management, thus negating any association. Nevertheless, COPD has not been included in pre-operative cardiac risk indices, such as Goldman, Detsky, and Lee and, indeed, no improvement was found in the prognostic value of the Lee index in vascular surgery patients when COPD was included. ${ }^{191}$ For PAH, on the other hand, the condition is so uncommon that its inclusion in an integrated risk model has not been considered.

In patients with pulmonary disease having non-cardiac surgery, the treatment goals pre-operatively are to optimize pulmonary function and minimize respiratory complications. For COPD, treatment goals would include eliminating active infection with antibiotics; minimizing wheeze associated with any reversible disease using inhaled bronchodilators or steroids; reducing right and LV failure with diuretics; ensuring adequate oxygenation; and, finally, encouraging smoking cessation prior to surgery. In relation to peri-operative cardiac management, patients with COPD should be managed in the same way as those without COPD and, in particular, there 
are no special contra-indications to the use of cardioselective $\beta$-blockers or statins in COPD patients. ${ }^{93,192}$

$\mathrm{PAH}$ is incurable and the treatment goal is to reduce symptoms, and improve exercise capacity and right ventricular function. Anaesthesia and surgery may be complicated by acute right heart failure due to increase of pulmonary vascular resistance related to the impairment of lung ventilation, typical of the operative and post-operative state of thoracic and abdominal surgery. Specific drug therapy for PAH includes calcium channel blockers (only for the few patients who are responders to the acute vasoreactivity test), prostanoids, endothelin receptor antagonists, and phosphodiesterase type-5 inhibitors. ${ }^{143,193}$ Ideally, patients with PAH should have an optimized treatment regimen before any surgical intervention. It is recommended also that PAH-specific drug therapy is not withheld for $>12 \mathrm{~h}$ due to the perioperative fasting state. In case of progression of right heart failure in the post-operative period, it is recommended that the diuretic dose be optimized and, if necessary, that inotropic support with dobutamine be initiated. The role of starting new specific PAH drug therapy in the perioperative period has not been established. In the case of severe right heart failure, not responsive to supportive therapy, the administration of temporary inhaled nitric oxide or i.v. epoprostenol with the guidance of a physician experienced in the treatment of PAH may be indicated. In this case, a period of progressive weaning from these medications may be required.

Patients with COPD and PAH have a relatively high frequency of heart failure and coronary heart disease. There is no consistent evidence indicating that COPD patients are at higher risk of perioperative cardiac complications and death, so that they can be managed in the same way as patients without COPD. On the other hand, PAH increases perioperative risk, and requires pre-operative assessment and, if severe, perioperative treatment.

\section{Recommendations on pulmonary diseases}

\begin{tabular}{|c|c|c|}
\hline Recommendations & Class $^{a}$ & Level $^{\mathrm{b}}$ \\
\hline $\begin{array}{l}\text { It is recommended that patients with pulmonary arterial } \\
\text { hypertension have an optimized treatment regimen before } \\
\text { any surgical intervention }\end{array}$ & 1 & $\mathrm{C}$ \\
\hline $\begin{array}{l}\text { In case of progression of right heart failure in the post- } \\
\text { operative period of patients with pulmonary arterial } \\
\text { hypertension, it is recommended the diuretic dose be } \\
\text { optimized and if necessary that inotropic support with } \\
\text { dobutamine be initiated }\end{array}$ & 1 & $\mathrm{C}$ \\
\hline $\begin{array}{l}\text { In the case of severe right heart failure not responsive } \\
\text { to supportive therapy the temporary administration of } \\
\text { inhaled nitric oxide or i.v. epoprostenol may be } \\
\text { considered with the guidance of a physician } \\
\text { experienced in the treatment of pulmonary arterial } \\
\text { hypertension }\end{array}$ & $\mathrm{llb}$ & $\mathrm{C}$ \\
\hline $\begin{array}{l}\text { Special perioperative cardiac risk management for } \\
\text { patients with COPD is not recommended }\end{array}$ & III & $\mathrm{C}$ \\
\hline
\end{tabular}

\section{Perioperative monitoring Electrocardiography}

Although even a single post-operative ECG demonstrating ischaemia in the recovery room is predictive of a major cardiac complication later during the hospital stay, ECG monitoring alone is not adequate to detect ischaemia in real time in the intensive care unit (ICU) and intraoperative settings. ${ }^{194-196}$ Specifically, conventional visual ECG monitoring for the detection of transient STsegment changes is inaccurate. ${ }^{196}$ Although lead $V_{5}$ has been known as the best choice for the detection of intraoperative ischaemia for many years, ${ }^{197,198}$ one study found that lead $V_{4}$ was more sensitive and appropriate than lead $V_{5}$ for detecting prolonged post-operative ischaemia and infarction. ${ }^{199}$ Leads are not specific for ischaemic events, and, furthermore, ischaemic events are dynamic and may not always appear in the same lead. If a single lead is used for monitoring, there is an increased risk of missing ischaemic events. With the use of selected lead combinations, more ischaemic events can be precisely diagnosed in the intraoperative setting. In one study, although the best sensitivity was obtained with lead $\mathrm{V}_{5}(75 \%)$, followed by lead $\mathrm{V}_{4}(61 \%)$, combining leads $V_{4}$ and $V_{5}$ increased the sensitivity to $90 \% .{ }^{198}$ In the same study, when three leads (II, $\mathrm{V}_{4}$, and $\mathrm{V}_{5}$ ) were used simultaneously, the sensitivity increased to $96 \% .{ }^{198}$ Similarly, in another study in which two or more precordial leads were used, the sensitivity of ECG monitoring was $>95 \%$ for detection of perioperative ischaemia and infarction. ${ }^{199}$ It was also shown that ECG monitoring with fewer leads (as few as three leads) had lower sensitivity than monitoring with 12 leads, and there was a statistically significant association, independent of perioperative troponin values, between perioperative ischaemia on a 12-lead ECG and long-term mortality. ${ }^{200-202}$ Thus, 12-lead ECG monitoring is recommended especially with high-risk patients.

ST-segment monitoring has been shown to be limited in patients who have intraventricular conduction defects (e.g. left bundle branch block) and ventricular paced rhythms. ${ }^{203}$ The secondary ST-T changes, which were present in these patients, were due to abnormal depolarization, which also distorted the repolarization process. The distorted ST-segments can limit the sensitivity of the ST-segment monitoring system. ${ }^{203}$ Because detection of ST-segment changes of the electrocardiogram by visual inspection is poor, computerized analysis has become standard in modern monitors. Continuous automated ST trending monitors are included in most new operating room ECG monitors to facilitate ischaemia detection. Such devices increase the sensitivity of ECG ischaemia detection. ${ }^{196}$ In one study, Holter recordings were used as the reference standard for detection of intraoperative ischaemia, and the ST trending monitors were found to have overall sensitivity and specificity of 74 and $73 \%$, respectively. Several conditions contributed 
to the inaccuracy of ST trend monitoring, and additional modification of their performance was necessary to achieve better agreement with the Holter analysis. ${ }^{196}$

In a series of studies during the past decade, the presence of ECG changes during monitoring in high-risk cohorts has been linked to a higher incidence of perioperative MI and cardiac events. In addition, the duration of STsegment changes positively correlates with the incidence of perioperative MI. ${ }^{204}$ Therefore, when ST-segment changes occur, the clinician should assume that myocardial ischaemia is present. ${ }^{205}$ However, it is not clear if ECG monitoring is sufficiently sensitive to identify patients at low risk. ${ }^{206,207}$ In addition, the usefulness of this test in the general population is limited because many studies have excluded patients with ECG findings that preclude accurate evaluation of ischaemia.

\begin{tabular}{lll} 
Recommendations on 12-lead ECG monitoring & & \\
\hline Recommendations & Class $^{\mathrm{a}}$ & Level $^{\mathrm{b}}$ \\
\hline $\begin{array}{l}\text { 12-lead ECG monitoring is recommended for all } \\
\text { patients undergoing surgery }\end{array}$ & $\mathrm{C}$ & $\mathrm{B}$ \\
$\begin{array}{l}\text { Selected lead combinations for better ischaemia } \\
\text { detection in operation room should be considered }\end{array}$ & Ila \\
\hline ECG, electrocardiograph. ${ }^{\text {a }}$ Class of recommendation. ${ }^{\mathrm{b}}$ Level of evidence. $^{\text {E }}$ &
\end{tabular}

\section{Transoesophageal echocardiography}

Transoesophageal echocardiography (TOE) has frequently been used as a monitoring tool during cardiac surgery since the mid-1980s. However, few evidencebased data support TOE use in non-cardiac surgery. TOE has several advantages over alternative monitoring methods, such as the use of a pulmonary artery catheter. It is rapidly available, relatively non-invasive, and provides more versatile and comprehensive information. However, although TOE is in general a safe procedure, serious adverse events can occur. The complication rates relate to the experience of the operator and the presence of severe oesophageal or gastric diseases. Specific training of users is mandatory to avoid inaccurate interpretation.

Myocardial ischaemia can be identified by abnormalities in regional wall motion and thickening. The concordance between intraoperative TOE and ECG is rather weak. ${ }^{208}$ Both ST-segment changes and regional wall motion abnormalities can be present in the absence of acute ischaemia. Wall motion abnormalities may be difficult to interpret in the presence of left bundle branch block, ventricular pacing, AF, or right ventricular overload. The resolution of ischaemia is not necessarily detectable if ischaemia is followed by myocardial stunning. Episodes of new or worsened wall motion abnormalities have been shown to be relatively infrequent $(20 \%)$ in high-risk patients undergoing non-cardiac surgery. ${ }^{208}$ They were more common in patients submitted to aortic vascular surgery. Episodes were poorly correlated with postoperative cardiac complications. ${ }^{208}$

When compared with pre-operative clinical data and intraoperative monitoring using 2-lead ECG, routine monitoring for myocardial ischaemia with TOE or 12lead ECG during non-cardiac surgery has little incremental clinical value in identifying patients at high risk of perioperative ischaemic outcomes. ${ }^{209}$

\begin{tabular}{|c|c|c|}
\hline Recommendations & Class $^{a}$ & Level $^{\mathrm{b}}$ \\
\hline $\begin{array}{l}\text { The use of TOE should be considered in patients who } \\
\text { develop ST-segment changes on intraoperative or } \\
\text { perioperative ECG monitoring }\end{array}$ & Ila & C \\
\hline $\begin{array}{l}\text { The use of TOE may be considered in patients at high } \\
\text { risk of developing myocardial ischaemia who undergo } \\
\text { major non-cardiac surgery }\end{array}$ & $\mathrm{llb}$ & C \\
\hline
\end{tabular}

ECG, electrocardiograph; TOE, transoesophageal echocardiography. ${ }^{\text {a Class of }}$ recommendation. ${ }^{\mathrm{b}}$ Level of evidence.

TOE is recommended if acute and severe haemodynamic instability or life-threatening abnormalities develop during or after surgery. ${ }^{210}$ The main advantage of TOE over pulmonary artery catheterization is the more comprehensive evaluation of cardiac structure and function. Information is quickly available on regional or global, right and/or LV dysfunction, the presence of tamponade or cardiac thrombi, and preload estimation through the measurement of end-diastolic volume. Numerous indices of ventricular and atrial function have been proposed. However, most parameters are load dependent.

The role of TOE for haemodynamic monitoring in patients at risk is more controversial. Automated analysis systems exist but are not yet sufficiently validated. There is no evidence that haemodynamic monitoring by TOE accurately stratifies risk or predicts outcome.

TOE can be useful in the operating room in patients with severe valvular lesions. The loading conditions during general anaesthesia differ from those present in the preoperative evaluation. Functional and ischaemic mitral regurgitation are usually reduced during general anaesthesia. Organic mitral regurgitation can, conversely, increase. In the setting of severe mitral regurgitation, the $L V$ ejection fraction overestimates $L V$ function, and other parameters may be more accurate, such as myocardial velocities or deformation obtained by tissue Doppler imaging or 2D speckle tracking, an angleindependent method. These are promising techniques, but more validation is needed before they can be used routinely in this setting. In patients with severe aortic stenosis, appropriate preload is important during surgery. Monitoring of $\mathrm{LV}$ end-diastolic volume may be more 
accurate than that of pulmonary capillary pressure. An appropriate heart rate is crucial in patients with mitral stenosis and aortic regurgitation: a long diastolic period in the former and shorter duration of diastole in the latter. When inappropriate control of heart rate occurs, the consequences should be assessed: changes in transmitral mean gradient and pulmonary arterial pressures in mitral stenosis and changes in $L V$ volumes and indices of $L V$ function in aortic regurgitation.

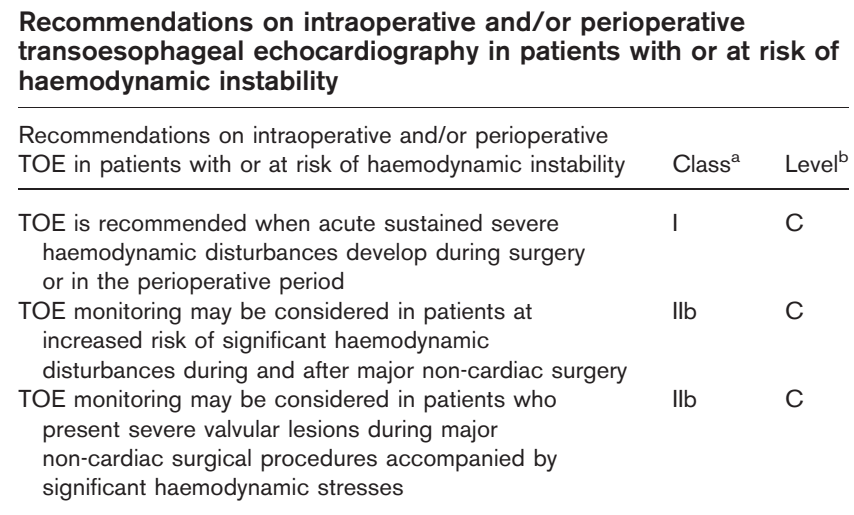

TOE, transoesophageal echocardiography. ${ }^{\text {a }}$ Class of recommendation. ${ }^{b}$ Level of evidence.

\section{Right heart catherization}

Most post-operative ischaemic episodes are silent and not accompanied by changes in pulmonary capillary wedge pressure. Right heart catheterization is not recommended for monitoring patients with intraoperative ischaemia. Indeed, both a large observational study and a randomized multicentre clinical trial did not show a benefit associated with the use of right heart catheterization after major non-cardiac surgery. ${ }^{211,212} \mathrm{~A}$ case-control analysis was carried out on a subset of patients from the observational study who underwent pulmonary artery catheter placement and who were matched with a similar number of patients who did not undergo right heart catheterization. Patients, who were adjusted for surgical procedure and propensity of catheterization, demonstrated a higher incidence of post-operative heart failure and non-cardiac events in the group submitted to catheterization. ${ }^{211}$

In the randomized study, no difference in mortality and hospital duration was found, but patients submitted to right heart catheterization had a higher incidence of pulmonary embolism. ${ }^{212}$

\section{Disturbed glucose metabolism}

Diabetes mellitus is an important risk factor for perioperative cardiac complications and death. This condition promotes atherosclerosis, endothelial dysfunction, and activation of platelets and proinflammatory cytokines. Surgical stress is associated with haemodynamic stress and vasospasm and further enhances the prothrombotic state, while inhibiting fibrinolysis. This may lead to instability of pre-existing coronary plaques, thrombus formation, vessel occlusion, and MI. Also, hyperglycaemia in the absence of established diabetes plays an important role, emphasizing the need for pre-operative management of hyperglycaemia where possible. This is illustrated by studies on patients with pre-diabetes glucose levels who undergo non-cardiac vascular or non-vascular surgery, showing $\sim 2$ - to 4-fold increases in risk of myocardial ischaemia, troponin release, 30-day and long-term cardiac events, and risk of death or cardiovascular mortality in particular. ${ }^{23,214}$ Importantly, impaired glucose tolerance is often identified only after glucose loading. Critical illness is another condition characterized by disturbed glucose homeostasis ('stress diabetes' or 'diabetes of injury'), which develops independent of previously diagnosed diabetes and has repeatedly been identified as an important risk factor for morbidity and/or mortality.

Data from the International Diabetes Foundation reveal a high and increasing prevalence of diabetes in Europe, rising from $7.8 \%$ in 2003 to $8.4 \%$ in 2007 , with an estimated prevalence of at least $9.1 \%$ by $2025 .{ }^{215}$ More than $30 \%$ of the cases were previously undiagnosed, pointing to underestimation of the problem. With $\sim 48$ million people affected, diabetes has become one of the main causes of morbidity and mortality in Europe. According to the World Health Organization, 50\% of these patients die of CVDs. It has been well established that surgery in patients with diabetes is associated with longer hospital stay, higher healthcare resource utilization, and greater perioperative mortality. More recently, the emphasis has shifted from diabetes to hyperglycaemia on its own. New-onset hyperglycaemia, as compared with hyperglycaemia in known diabetics, may hold a much higher risk of adverse outcome. ${ }^{216}$

Evidence for strict blood glucose control for patients without known diabetes undergoing non-cardiac surgery is largely derived from studies in critically ill patients. ${ }^{217}$ In 2001 the landmark Leuven prospective randomized controlled study demonstrated major clinical benefits for surgical ICU patients whose blood glucose levels were maintained normal $(5.0-5.6 \mathrm{mmol} / \mathrm{L} ; 90-100 \mathrm{mg} / \mathrm{dL})$ with intensive insulin therapy, compared with patients who received conventional glucose management and developed hyperglycaemia $(8.3-8.9 \mathrm{mmol} / \mathrm{L} ; 150$ $160 \mathrm{mg} / \mathrm{dL}){ }^{218}$ These benefits included lower ICU and in-hospital mortality and prevention of several critical illness-associated complications (critical illness polyneuropathy, severe infections, acute renal failure, and prolonged dependency on mechanical ventilation and intensive care). Also, long-term outcome improved, as shown for the cardiac surgery subgroup. Five years later the Leuven group reported findings from the medical 
ICU, showing prevention of morbidity, but no mortality benefit from intensive glucose control, except in a subgroup requiring critical care for 3 days. ${ }^{219}$ Based on these two trials recommendations were made aiming at tight glucose control. Several observational implementation studies on tight glucose management or small, randomized studies in selected ICU patient groups supported the clinical benefits of the Leuven studies. ${ }^{217}$ Pooled analysis of the Leuven studies revealed reduced mortality and morbidity for all major clinical diagnostic subgroups, including cardiovascular, respiratory, gastrointestinal/ hepatic disease or surgery, active malignancy, and sepsis upon ICU admission. Patients with known diabetes tended to experience less morbidity but a survival benefit appeared absent. All studies described above started glucose control after ICU admission. Timing of initiating insulin therapy is controversial, but a recent medical ICU study showed better outcome when initiated within the first $48 \mathrm{~h}$ than after $48 \mathrm{~h}$. Tight intraoperative glucose control may provide additional benefit but appears a challenge and, so far, studies have mainly been set up for cardiac surgery. Moderate intraoperative glycaemic control during CABG (not continued in the ICU) resulted in decreased need for pacing, lower incidence of AF and infections, shortening of the ICU and hospital stay, and decreased recurrent ischaemic events in the long-run. In contrast, implementation of glycaemic control during cardiac surgery, superimposed upon post-operative ICU glycaemic control, did not further reduce perioperative mortality or morbidity. ${ }^{220}$ In an observational study, stricter glucose control during liver transplantation was associated with a lower infection rate and 1-year mortality than poor glycaemic control. ${ }^{221}$

Studies in the field of critical care have demonstrated the detrimental effect of hyperglycaemia, due to an adverse effect on renal and hepatic function, endothelial function, and immune response, particularly in patients without underlying diabetes. In the Leuven studies, risk of death and degree of hyperglycaemia were positively correlated. Unequivocal demonstration that glycaemic control rather than direct insulin effects mediated the survival and most morbidity benefits of insulin therapy was provided in a rabbit model of critical illness. ${ }^{222}$ Several risk factors for cardiac events after non-cardiac surgery are attenuated with strict blood glucose control in the ICU, including endothelial injury/dysfunction, CRP, and asymmetric dimethylarginine, apart from effects on mitochondrial damage, serum lipid profile, and the cortisol response. No effects, or only marginal ones, were seen on cytokines, coagulation, and fibrinolysis.

Recently, the favourable outcomes of the Leuven findings using tight glucose control were questioned. The Normoglycaemia in Intensive Care Evaluation and Survival Using Glucose Algorithm Regulation (NICESUGAR) study investigators randomized $>6000$ patients
(63\% medical ICU and 37\% surgical ICU) to either tight glucose control (target glucose level, $4.5-6.0 \mathrm{mmol} / \mathrm{L}$; $81-108 \mathrm{mg} / \mathrm{dL}$ ) or conventional glucose control (target glucose level, $8.0-10.0 \mathrm{mmol} / \mathrm{L} ; \quad 144-180 \mathrm{mg} / \mathrm{dL}){ }^{223}$ Patients were randomized to treatment within $24 \mathrm{~h}$ after admission using i.v. insulin infusions for glucose control. The primary endpoint, death by 90 days after randomization, was increased with intensive glucose control (27.5\%) as compared with $24.9 \%$ with conventional control. There was no morbidity difference between the two study groups, and hence the excess mortality remains unexplained. As could be expected, hypoglycaemia $(<40 \mathrm{mg} / \mathrm{dL})$ occurred in more patients in the intensive-control group than in the conventional-control group (6.8\% vs. $0.5 \%, P<0.001)$. The strength of the NICESUGAR trial was its large and multicentre design using a computer-guided insulin infusion protocol. However, this protocol used an if-then algorithm based upon inaccurate and non-standardized stand-alone glucometers for blood glucose measurements. In addition, NICE-SUGAR had an open-label design, a small imbalance between the groups with respect to corticosteroid therapy, and $10 \%$ of patients randomized to intensive glucose control discontinued the study prematurely. The differences in outcome between the two studies should be explained.

(i) The Leuven trials were performed in a single centre with standardized care which included early parenteral nutrition supplementing enteral feeding, whereas in the NICE-SUGAR trial enteral nutrition predominated, resulting in hypocaloric feeding in particular during the first week after admission to ICU.

(ii) The target for initiating insulin in the standard treatment group was different, with insulin being advocated in the Leuven study only when blood glucose exceeded the renal threshold of $>215 \mathrm{mg} / \mathrm{dL}$, an approach that considers hyperglycaemia as a possible beneficial adaptation, whereas in NICESUGAR a target of $144-180 \mathrm{mg} / \mathrm{dL}$ was used in the standard group, which resulted in $70 \%$ of the patients receiving insulin and reaching an average blood glucose level of $8.0 \mathrm{mmol} / \mathrm{L}(144 \mathrm{mg} / \mathrm{dL})$.

(iii) Also in the intervention group of NICE-SUGAR, the compliance to therapy was much lower than in the Leuven studies, which resulted in an average glucose level of $6.6 \mathrm{mmol} / \mathrm{L}(118 \mathrm{mg} / \mathrm{dL})$ and a very large overlap with the glucose levels in the control group.

(iv) The use of inaccurate glucometers in NICESUGAR may have misguided the insulin therapy and may have overlooked hypokalaemia, a possible cause of excess cardiovascular mortality, which is prevented with the use of blood gas analysers for glucose measurement.

(v) The nurse experience with the intervention in NICE-SUGAR was much less than in the Leuven studies, in view of the limited number of patients 
Table 10 Clinical benefits of intensive insulin therapy in critically ill patients with a non-cardiac diagnosis upon ICU admission ${ }^{218,219}$

\begin{tabular}{lccr}
\hline & \multicolumn{2}{c}{ ICU stay $\geq 3$ days } & \\
\cline { 2 - 3 } & CIT $(n=643)$ & IIT $(n=648)$ & $P$-value \\
\hline ICU mortality & $27.4 \%$ & $22.7 \%$ & 0.05 \\
In-hospital mortality & $38.7 \%$ & $32.1 \%$ & 0.01 \\
Renal replacement therapy & $11.2 \%$ & $7.3 \%$ & 0.02 \\
Critical illness polyneuropathy $^{\mathrm{a}}$ & $51.3 \%$ & $34.4 \%$ & $<0.01$ \\
Bacteraemia $^{\text {Mechanical ventilation (days) }}{ }^{\mathrm{b}}$ & $13.5 \%$ & $10.6 \%$ & 0.11 \\
ICU stay (days) $^{\mathrm{b}}$ & $8(4-17)$ & $7(3-13)$ & 0.01 \\
& $9(4-18)$ & $8(4-15)$ & 0.05
\end{tabular}

CIT, conventional insulin therapy; ICU, intensive care unit; IIT, intensive insulin therapy. ${ }^{\text {a }}$ Percentage of those screened. ${ }^{b}$ Median (interquartile range).

recruited per centre $(<15 \%$ of all patients screened in the participating ICUs) as compared with $70-95 \%$ in the Leuven studies.

The results of the NICE-SUGAR trial may suggest that intensive glucose control could harm patients admitted to the ICU, in terms of death, when glucose levels are below the range of $7.8-10.0 \mathrm{mmol} / \mathrm{L}(140-180 \mathrm{mg} / \mathrm{dL})$. In contrast, evidence derived from previous studies suggests the clinical benefit of maintenance of normoglycaemia (4.4$6.1 \mathrm{mmol} / \mathrm{L} ; 80-110 \mathrm{mg} / \mathrm{dL}$ ) as compared with tolerating hyperglycaemia up to $11.9 \mathrm{mmol} / \mathrm{L}(215 \mathrm{mg} / \mathrm{dL})$ for adult critically ill patients (Table 10).

Until further data become available clarifying the reasons for the different outcomes between the studies, it is recommended that the management of blood glucose in the ICU be optimized, avoiding the extremes of hyperglycaemia and also hypoglycaemia. The available data indicate that this therapy should be started immediately after ICU admission. It may be advisable to target a level of $\sim 8.0 \mathrm{mmol} / \mathrm{L}(144 \mathrm{mg} / \mathrm{dL})$ for settings and patient populations that are comparable with those studied in NICE-SUGAR.

\section{Recommendations on blood glucose control}

\begin{tabular}{lll}
\hline Recommendations & Class $^{\mathrm{a}}$ & Level $^{\mathrm{b}}$ \\
\hline $\begin{array}{l}\text { Post-operative prevention of hyperglycaemia [targeting } \\
\text { levels at least below } 10.0 \mathrm{mmol} / \mathrm{L}(180 \mathrm{mg} / \mathrm{dL})] \text { with }\end{array}$ & $\mathrm{I}$ & $\mathrm{B}$ \\
$\begin{array}{l}\text { intensive insulin therapy is recommended in adults } \\
\text { after high-risk or complicated major surgery requiring }\end{array}$ & & \\
$\begin{array}{l}\text { admission to ICU } \\
\begin{array}{l}\text { Intraoperative prevention of hyperglycaemia with insulin } \\
\text { may be considered }\end{array}\end{array}$ \\
$\begin{array}{l}\text { Post-operative prevention of hyperglycaemia with insulin } \\
\text { after uncomplicated elective surgery may be considered }\end{array}$ & $\mathrm{llb}$ & $\mathrm{C}$ \\
\hline
\end{tabular}

$\mathrm{ICU}$, intensive care unit. ${ }^{\mathrm{a}}$ Class of recommendation. ${ }^{\mathrm{b}}$ Level of evidence.

\section{Anaesthesia}

An optimal perioperative course stems from a close cooperation between cardiologists, surgeons, pulmonologists, and anaesthesiologists. Pre-operative risk assess- ment and pre-operative optimization of cardiac disease should be performed jointly.

There is a paucity of strong evidence-based data supporting the choice of a particular perioperative approach and thus several options are available. Sufficiently powered randomized trials addressing the potential relationship between patient outcome and perioperative management are still lacking for cardiac patients undergoing noncardiac surgery.

\section{Intraoperative anaesthetic management}

The choice of the anaesthetic agent has been considered to be of little importance with regard to patients' outcome provided the vital functions are adequately supported. There is conflicting evidence from cardiac surgery over whether a specific method is advantageous in cardiac disease, but there is no evidence of superiority of any specific anaesthetic agent in non-cardiac surgery. ${ }^{224,225}$

Most anaesthetic techniques reduce sympathetic tone, leading to vasodilatation and reduction in systemic blood pressure. Thus, anaesthesiological management must ensure the proper maintenance of organ perfusion pressure.

\section{Neuraxial techniques}

Spinal and epidural anaesthesia also induce sympathetic blockade. Depending on the height of the block, it induces peripheral vasodilation with fall in blood pressure. When reaching the thoracic dermatome level 4, a reduction in cardiac sympathetic drive with subsequent reduction in myocardial contractility, heart rate, and change in cardiac loading conditions will appear. The speed and strength of sympathetic blockade will depend on dosage and drugs as well as the patient's condition. There is conflicting evidence on the effect of neuraxial blocks on patient outcome after non-cardiac surgery. One meta-analysis reported significantly improved survival and reduced incidence of post-operative thromboembolic, cardiac and pulmonary complications with neuraxial blockade compared with general anaesthesia. ${ }^{226} \mathrm{~A}$ major criticism of this study has been the inclusion of older studies, which may have made the results invalid for current practice. A recent analysis of a large cohort of patients (10564 patients without and 2253 patients with epidural) undergoing colon resection confirmed the improved survival with epidural analgesia at 7 and 30 days after surgery, but it was not possible to identify the cause of death. ${ }^{227}$ Also cardiac morbidity was not different between the two groups.

Randomized studies and a meta-analysis of several randomized clinical trials in non-cardiac surgery patients, comparing outcome with regional and general anaesthetic 
techniques, have shown little consistent evidence of improved outcome and reduced post-operative morbidity and mortality. ${ }^{228-230}$ It has been estimated that the number of patients needed for a randomized clinical trial to determine whether epidural anaesthesia and analgesia would affect mortality in patients undergoing high-risk vascular surgery would be $\sim 24000$, while enrolment of 1.2 million would be needed in a lowrisk procedure. ${ }^{227}$ Thus, present studies are underpowered for a valid analysis of risk of death for procedures with low surgical risk. No study has clearly demonstrated a difference in outcome with different monitoring techniques, fluid management, or transfusion strategies. Most studies have used different pre-determined therapeutic goals, often requiring inotropic support, a factor that may have been of importance for the results. ${ }^{212}$ The importance of skilled anaesthesiological management in keeping adequate circulation is often underlined. ${ }^{231}$

\section{Post-operative pain management}

Post-operative pain is a major concern, reported in $5-10 \%$ of the patients. It may increase sympathetic drive and delay recovery. ${ }^{232,233}$ The evidence that pain causes organ complications after surgery is less clear. Neuraxial analgesia with local anaesthetics/opioids and/or $\alpha_{2}$-agonists, i.v. opioids alone or in combination with non-steroidal anti-inflammatory drugs seems to be the most effective. The benefit of invasive analgesic techniques should be weighed against potential dangers. This is of special importance when considering the use of neuraxial blockade in patients under chronic antithrombotic therapy due to increased potential of a neuraxial haematoma. It is beyond the scope of these guidelines to give recommendations for the use of neuraxial blocks in patients with coagulation disturbances.

Patient-controlled analgesia is an alternative for postoperative pain relief. Recent meta-analyses of controlled randomized trials show that patient-controlled analgesia has some advantage with regard to patient satisfaction over nurse-controlled or on-demand analgesia. ${ }^{234}$ No difference with regard to morbidity or final outcome was demonstrated. Patient-controlled analgesia is an adequate alternative in patients and situations not suited for regional anaesthesia. Routines for follow-up and documentation of effects should be in place. ${ }^{232,235-237}$ Non-steroidal anti-inflammatory drugs and the cyclooxygenase-2 (COX-2) inhibitors have the potential for promoting heart and renal failure as well as thromboembolic events and should be avoided in patients with myocardial ischaemia. The COX-2 inhibitors cause less gastrointestinal ulceration and bronchospasm. The final role for these drugs in the treatment of post-operative pain in cardiac patients undergoing non-cardiac surgery has not been defined. The drugs should be avoided in patients with renal and heart failure, elderly patients, patients on diuretics, as well as patients with unstable haemodynamics. ${ }^{238}$

\begin{tabular}{lll} 
Recommendations on anaesthesia & \\
\hline Recommendations & Class $^{\mathrm{a}}$ & Level $^{\mathrm{b}}$ \\
\hline $\begin{array}{l}\text { Consideration should be given to performing thoracic } \\
\text { epidural anaesthesia in high-risk surgery for patients } \\
\text { with cardiac disease }\end{array}$ & A Ila \\
Use of non-steroidal anti-inflammatory drugs and \\
COX-2 inhibitors for post-operative pain control \\
is not recommended in patients with renal and \\
heart failure, myocardial ischaemia, elderly patients, \\
as well as in patients taking diuretics or having \\
unstable haemodynamics
\end{tabular}

COX-2, cyclooxygenase-2. ${ }^{a}$ Class of recommendation. ${ }^{b}$ Level of evidence.

\section{Putting the puzzle together}

Figure 4 presents in algorithmic form an evidence-based stepwise approach for determining which patient benefits from cardiac testing, coronary artery revascularization, and cardiovascular therapy prior to surgery. For each step the committee has included the level of the recommendations and the strength of evidence in the accompanying Table 11.

Step 1. The urgency of the surgical procedure should be assessed. In urgent cases, patient- or surgical-specific factors dictate the strategy, and do not allow further cardiac testing or treatment. In these cases, the consultant provides recommendations on perioperative medical management, surveillance for cardiac events, and continuation of chronic cardiovascular medical therapy.

Step 2. If the patient is unstable, as presented in Table 12, this condition should be clarified and treated appropriately prior to surgery. Examples are unstable coronary syndromes, decompensated heart failure, severe arrhythmias, or symptomatic valvular disease. This usually leads to cancellation or delay of the surgical procedure. For instance, patients with unstable angina pectoris should be referred for coronary angiography to assess the therapeutic options. Treatment options should be discussed in a multidisciplinary team, involving all perioperative care physicians, because interventions might have implications for anaesthesiological and surgical care. For example, the initiation of dual antiplatelet therapy after coronary artery stent placement might complicate loco-regional anaesthesia or specific surgical procedures. Depending on the outcome of this discussion, patients can proceed for coronary artery intervention, namely CABG, balloon angioplasty, or stent placement with the initiation of dual antiplatelet therapy if the index surgical procedure can be delayed, or directly for operation if delay is incompatible with optimal medical therapy. 
Fig. 4

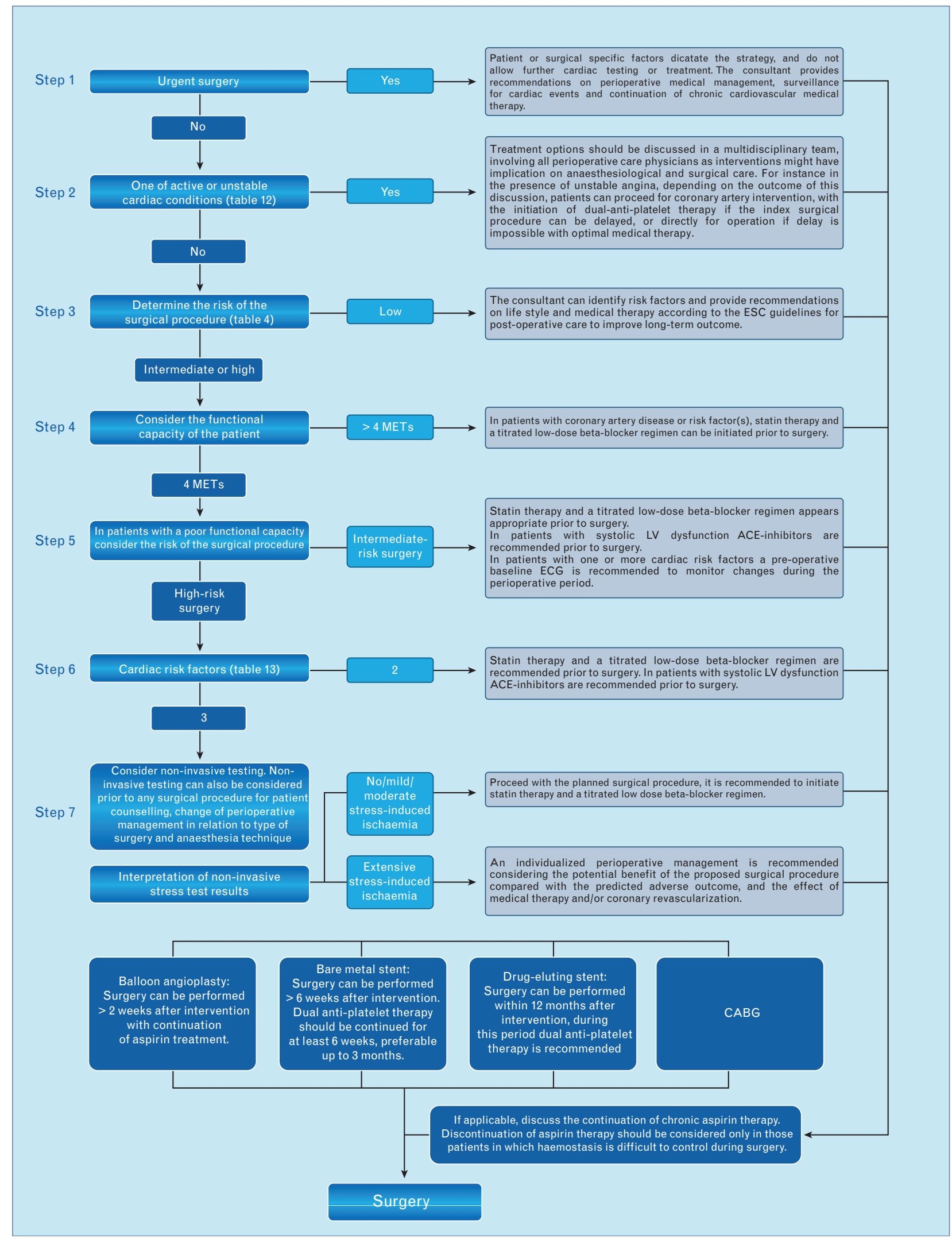

Summary of pre-operative cardiac risk evaluation and perioperative management. 


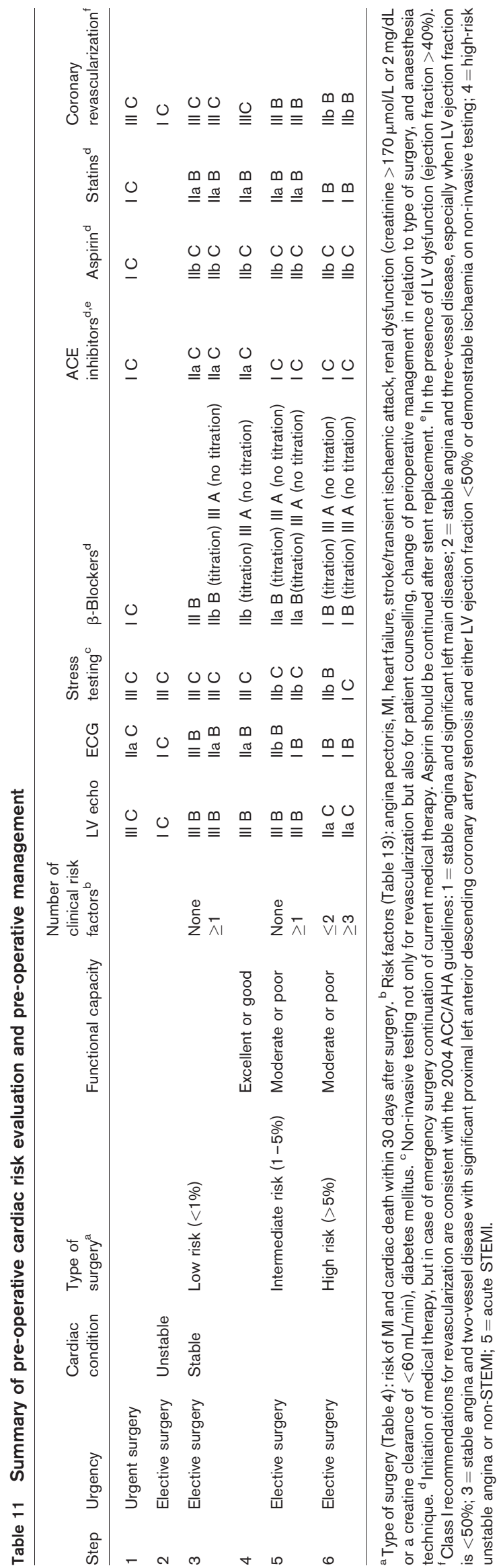

\section{Table 12 Unstable cardiac conditions}

Unstable angina pectoris

Acute heart failure

Significant cardiac arrhythmias

Symptomatic valvular heart disease

Recent $\mathrm{Ml}^{\mathrm{a}}$ and residual myocardial ischemia

${ }^{\text {a }} \mathrm{An} \mathrm{Ml}$ within 30 days, according to the universal definition of $\mathrm{Ml}^{34}$

Step 3. Determine the risk of the surgical procedure (Table 4). If the estimated 30-day cardiac risk of the procedure in cardiac-stable patients is low, $<1 \%$, it is unlikely that test results will change management and it would be appropriate to proceed with the planned surgical procedure. The consultant can identify risk factors and provide recommendations on lifestyle and medical therapy according to the ESC Guidelines for post-operative care to improve long-term outcome.

Step 4. Consider the functional capacity of the patient. If an asymptomatic or cardiac-stable patient has moderate or good functional capacity, $>4$ METs, perioperative management is unlikely to be changed on the basis of test results irrespective of the planned surgical procedure. Even in the presence of clinical risk factors, it is appropriate to refer the patient for surgery. In patients with IHD or risk factor(s), statin therapy and a titrated low-dose $\beta$-blocker regimen can be initiated prior to surgery, as outlined in Table 11.

Step 5. It is recommended that chronic aspirin therapy be continued. Discontinuation of aspirin therapy should be considered only in those patients in which haemostasis is difficult to control during surgery.

Step 6. In patients with a moderate or poor functional capacity, consider the risk of the surgical procedure, as outlined in Table 4. Patients scheduled for intermediaterisk surgery can proceed for surgery; statin therapy and a titrated low-dose $\beta$-blocker regimen appears appropriate prior to surgery. In patients with systolic $\mathrm{LV}$ dysfunction, evidenced by $\mathrm{LV}$ ejection fraction $<40 \%$, ACE inhibitors (or ARBs in patients intolerant of ACE inhibitors) are recommended before surgery. In patients with one or more clinical risk factors, a pre-operative baseline ECG is recommended to monitor changes during the perioperative period. In patients scheduled for high-risk surgery, as described in Table 4, clinical risk factors (Table 13) are noted. In patients with up to two clinical

Table 13 Clinical risk factors

Angina pectoris

Prior $\mathrm{Ml}^{\mathrm{a}}$

Heart failure

Stroke/transient ischaemic attack

Renal dysfunction (serum creatinine $>170 \mu \mathrm{mol} / \mathrm{L}$ or $2 \mathrm{mg} / \mathrm{dL}$ or a creatinine

clearance of $<60 \mathrm{~mL} / \mathrm{min}$ )

Diabetes mellitus requiring insulin therapy

${ }^{\text {a }}$ According to the universal definition of $\mathrm{Ml}^{34}$ 
risk factors, statin therapy and a titrated low-dose $\beta$-blocker regimen are recommended prior to surgery. In patients with systolic $\mathrm{LV}$ dysfunction, evidenced by $\mathrm{LV}$ ejection fraction $<40 \%$, ACE inhibitors (or ARBs in patients intolerant of $\mathrm{ACE}$ inhibitors) are recommended before surgery.

Consider non-invasive testing in patients with $\geq 3$ clinical risk factors (Table 13). Non-invasive testing can also be considered prior to any surgical procedure for patient counselling, or change of perioperative management in relation to type of surgery and anaesthesia technique.

Step 7. Interpretation of non-invasive stress test results. Patients without stress-induced ischaemia, or mild to moderate ischaemia suggestive of one- or two-vessel disease, can proceed with the planned surgical procedure. It is recommended that statin therapy and a titrated lowdose $\beta$-blocker regimen be initiated. In patients with extensive stress-induced ischaemia, as assessed by noninvasive testing, individualized perioperative management is recommended, taking into consideration the potential benefit of the proposed surgical procedure compared with the predicted adverse outcome. Also, the effect of medical therapy and/or coronary revascularization must be assessed, not only for immediate postoperative outcome, but also for long-term follow-up. In patients referred for percutaneous coronary artery intervention, the initiation and duration of antiplatelet therapy will interfere with the planned surgical procedure. In patients referred for angioplasty, non-cardiac surgery can be performed within 2 weeks after intervention with continuation of aspirin treatment. In patients with bare metal stent placement, non-cardiac surgery can be performed after 6 weeks to 3 months following intervention. Dual antiplatelet therapy should be continued for at least 6 weeks, preferably for up to 3 months. After this period, at least aspirin therapy should be continued. In patients with recent DES placement, non-cardiac surgery can be performed after 12 months following intervention, before which time dual antiplatelet therapy is recommended. After this period, at least aspirin therapy should be continued.

The CME text 'Guidelines for pre-operative cardiac risk assessment and perioperative cardiac management in non-cardiac surgery' is accredited by the European Board for Accreditation in Cardiology (EBAC). EBAC works according to the quality standards of the European Accreditation Council for Continuing Medical Education (EACCME), which is an institution of the European Union of Medical Specialists (UEMS). In compliance with EBAC/EACCME guidelines, all authors participating in this programme have disclosed potential conflicts of interest that might cause a bias in the article. The Organizing Committee is responsible for ensuring that all potential conflicts of interest relevant to the programme are declared to the participants prior to the CME activities.

CME questions for this article are available at: European Heart Journal http:// cme.oxfordjournals.org/cgi/hierarchy/oupcme_node;ehj and European Society of Cardiology http://www.escardio.org/guidelines.

\section{References}

1 Schwartz PJ, Breithardt G, Howard AJ, Julian DG, Rehnqvist Ahlberg N. Task Force Report: the legal implications of medical guidelines-a Task Force of the European Society of Cardiology. Eur Heart J 1999; 20:1152-1157.

2 Almanaseer Y, Mukherjee D, Kline-Rogers EM, Kesterson SK, Sonnad SS, Rogers B, Smith D, Furney S, Ernst R, McCort J, Eagle KA. Implementation of the ACC/AHA guidelines for preoperative cardiac risk assessment in a general medicine preoperative clinic: improving efficiency and preserving outcomes. Cardiology 2005; 103:24-29.

3 Poldermans D, Hoeks SE, Feringa $\mathrm{HH}$. Pre-operative risk assessment and risk reduction before surgery. J Am Coll Cardiol 2008; 51:1913-1924.

4 http://www.prismant.nl/. Ziekenhuisstatistiek-Verrichtingen. 2008, Prismant.

5 Lee TH, Marcantonio ER, Mangione CM, Thomas EJ, Polanczyk CA, Cook EF, Sugarbaker DJ, Donaldson MC, Poss R, Ho KK, Ludwig LE, Pedan A, Goldman L. Derivation and prospective validation of a simple index for prediction of cardiac risk of major noncardiac surgery. Circulation 1999; 100:1043-1049.

6 Boersma E, Kertai MD, Schouten O, Bax JJ, Noordzij P, Steyerberg EW, Schinkel AF, van Santen M, Simoons ML, Thomson IR, Klein J, van Urk H, Poldermans D. Perioperative cardiovascular mortality in noncardiac surgery: validation of the Lee cardiac risk index. Am J Med 2005; 118:1134-1141.

7 Poldermans D, Bax JJ, Kertai MD, Krenning B, Westerhout CM, Schinkel AF, Thomson IR, Lansberg PJ, Fleisher LA, Klein J, van Urk H, Roelandt JR, Boersma E. Statins are associated with a reduced incidence of perioperative mortality in patients undergoing major noncardiac vascular surgery. Circulation 2003; 107:1848-1851.

8 Poldermans D, Bax JJ, Schouten O, Neskovic AN, Paelinck B, Rocci G, van Dortmont L, Durazzo AES, van de Ven LLM, van Sambeek MRHM. Should major vascular surgery be delayed because of preoperative cardiac testing in intermediate-risk patients receiving beta-blocker therapy with tight heart rate control? J Am Coll Cardiol 2006; 48:964969.

9 Poldermans D, Boersma E, Bax JJ, Thomson IR, van de Ven LL, Blankensteijn JD, Baars HF, Yo TI, Trocino G, Vigna C, Roelandt JR, van Urk $\mathrm{H}$. The effect of bisoprolol on perioperative mortality and myocardial infarction in high-risk patients undergoing vascular surgery. Dutch Echocardiographic Cardiac Risk Evaluation Applying Stress Echocardiography Study Group. N Engl J Med 1999; 341:1789-1794.

10 Devereaux PJ, Yang H, Yusuf S, Guyatt G, Leslie K, Villar JC, Xavier D, Chrolavicius S, Greenspan L, Pogue J, Pais P, Liu L, Xu S, Malaga G, Avezum A, Chan M, Montori VM, Jacka M, Choi P. Effects of extendedrelease metoprolol succinate in patients undergoing non-cardiac surgery (POISE trial): a randomised controlled trial. Lancet 2008; 371:18391847.

11 Naughton C, Feneck RO. The impact of age on 6-month survival in patients with cardiovascular risk factors undergoing elective non-cardiac surgery. Int J Clin Pract 2007; 61:768-776.

12 Mangano DT. Perioperative medicine: NHLBI working group deliberations and recommendations. J Cardiothorac Vasc Anesth 2004; 18:1-6.

13 Ferguson TB Jr, Hammill BG, Peterson ED, DeLong ER, Grover FL. A decade of change-risk profiles and outcomes for isolated coronary artery bypass grafting procedures, 1990-1999: a report from the STS National Database Committee and the Duke Clinical Research Institute. Society of Thoracic Surgeons. Ann Thorac Surg 2002; 73:480-489; discussion 489-490.

14 Carroll K, Majeed A, Firth C, Gray J. Prevalence and management of coronary heart disease in primary care: population-based cross-sectional study using a disease register. J Public Health Med 2003; 25:29-35.

15 National Center for Health Statistics. Health, Unites States, 2007.

16 Hoeks SE, Scholte op Reimer WJ, Lenzen MJ, van Urk H, Jorning PJ, Boersma E, Simoons ML, Bax JJ, Poldermans D. Guidelines for cardiac management in noncardiac surgery are poorly implemented in clinical practice: results from a peripheral vascular survey in the Netherlands. Anesthesiology 2007; 107:537-544.

17 Hoeks SE, Scholte Op Reimer WJ, Schouten O, Lenzen MJ, van Urk H, Poldermans D. Statin use in the elderly: results from a peripheral vascular survey in The Netherlands. J Vasc Surg 2008; 48:891-895.

18 Hoeks SE, Scholte op Reimer WJM, van Gestel YRBM, Schouten O, Lenzen MJ, Flu W-J, van Kuijk J-P, Latour C, Bax JJ, van Urk H, Poldermans D. Medication underuse during long-term follow-up in patients with peripheral arterial disease. Circ Cardiovasc Qual Outcomes 2009:CIRCOUTCOMES.109.868505.

19 Wirthlin DJ, Cambria RP. Surgery-specific considerations in the cardiac patient undergoing noncardiac surgery. Prog Cardiovasc Dis 1998; 40:453-468. 
20 Fleisher LA, Beckman JA, Brown KA, Calkins $H$, Chaikof $E$ Fleischmann KE, Freeman WK, Froehlich JB, Kasper EK, Kersten JR, Riegel B, Robb JF, Smith SC Jr, Jacobs AK, Adams CD, Anderson JL, Antman EM, Buller CE, Creager MA, Ettinger SM, Faxon DP, Fuster V, Halperin JL, Hiratzka LF, Hunt SA, Lytle BW, Md RN, Ornato JP, Page RL, Riegel B, Tarkington LG, Yancy CW. ACC/AHA 2007 Guidelines on Perioperative Cardiovascular Evaluation and Care for Noncardiac Surgery: Executive Summary: A Report of the American College of Cardiology/American Heart Association Task Force on Practice Guidelines (Writing Committee to Revise the 2002 Guidelines on Perioperative Cardiovascular Evaluation for Noncardiac Surgery): Developed in Collaboration With the American Society of Echocardiography, American Society of Nuclear Cardiology, Heart Rhythm Society, Society of Cardiovascular Anesthesiologists, Society for Cardiovascular Angiography and Interventions, Society for Vascular Medicine and Biology, and Society for Vascular Surgery. Circulation 2007; 116:1971-1996.

21 Schouten O, Dunkelgrun M, Feringa HH, Kok NF, Vidakovic R, Bax JJ, Poldermans D. Myocardial damage in high-risk patients undergoing elective endovascular or open infrarenal abdominal aortic aneurysm repair. Eur J Vasc Endovasc Surg 2007; 33:544-549.

22 Blankensteijn JD, de Jong SE, Prinssen M, van der Ham AC, Buth J, van Sterkenburg SM, Verhagen HJ, Buskens E, Grobbee DE. Two-year outcomes after conventional or endovascular repair of abdominal aortic aneurysms. N Engl J Med 2005; 352:2398-2405.

23 Holte K, Kehlet H. Postoperative ileus: a preventable event. Br J Surg 2000; 87:1480-1493.

24 Nguyen NT, Wolfe BM. The physiologic effects of pneumoperitoneum in the morbidly obese. Ann Surg 2005; 241:219-226.

25 Gurusamy KS, Samraj K, Davidson BR. Abdominal lift for laparoscopic cholecystectomy. Cochrane Database Syst Rev 2008;(2):CD006574.

26 Hlatky MA, Boineau RE, Higginbotham MB, Lee KL, Mark DB, Califf RM Cobb FR, Pryor DB. A brief self-administered questionnaire to determine functional capacity (the Duke Activity Status Index). Am J Cardiol 1989; 64:651-654.

27 Fletcher GF, Balady GJ, Amsterdam EA, Chaitman B, Eckel R, Fleg J, Froelicher VF, Leon AS, Pina IL, Rodney R, Simons-Morton DA, Williams MA, Bazzarre T. Exercise standards for testing and training: a statement for health-care professionals from the American Heart Association. Circulation 2001; 104:1694-1740.

28 Biccard BM. Relationship between the inability to climb two flights of stairs and outcome after major non-cardiac surgery: implications for the pre-operative assessment of functional capacity. Anaesthesia 2005; 60:588-593.

29 Wiklund RA, Stein HD, Rosenbaum SH. Activities of daily living and cardiovascular complications following elective, noncardiac surgery. Yale J Biol Med 2001; 74:75-87.

30 Morris CK, Ueshima K, Kawaguchi T, Hideg A, Froelicher VF. The prognostic value of exercise capacity: a review of the literature. Am Heart $J$ 1991; 122:1423-1431.

31 Detsky AS, Abrams HB, Forbath N, Scott JG, Hilliard JR. Cardiac assessment for patients undergoing noncardiac surgery. A multifactorial clinical risk index. Arch Intern Med 1986; 146:2131-2134.

32 Goldman L, Caldera DL, Nussbaum SR, Southwick FS, Krogstad D, Murray B, Burke DS, O'Malley TA, Goroll AH Caplan CH, Nolan J, Carabello B, Slater EE. Multifactorial index of cardiac risk in noncardiac surgical procedures. N Engl J Med 1977; 297:845850.

33 Maisel AS, Bhalla V, Braunwald E. Cardiac biomarkers: a contemporary status report. Nat Clin Pract Cardiovasc Med 2006; 3:24-34.

34 Thygesen K, Alpert JS, White HD. Universal definition of myocardial infarction. Eur Heart J 2007; 28:2525-2538.

35 Sabatine MS, Morrow DA, Giugliano RP, Murphy SA, Demopoulos LA, DiBattiste PM, Weintraub WS, McCabe CH, Antman EM, Cannon CP, Braunwald E. Implications of upstream glycoprotein Ilb/llla inhibition and coronary artery stenting in the invasive management of unstable angina/ non-ST-elevation myocardial infarction: a comparison of the Thrombolysis In Myocardial Infarction (TIMI) IIIB trial and the Treat angina with Aggrastat and determine Cost of Therapy with Invasive or Conservative Strategy (TACTICS)-TIMI 18 trial. Circulation 2004; 109:874-880.

36 Cannon CP, McCabe CH, Wilcox RG, Langer A, Caspi A, Berink P, Lopez-Sendon J, Toman J, Charlesworth A, Anders RJ, Alexander JC, Skene A, Braunwald E. Oral glycoprotein Ilb/Illa inhibition with orbofiban in patients with unstable coronary syndromes (OPUS-TIMI 16) trial. Circulation 2000; 102:149-156.

37 Priebe HJ. Perioperative myocardial infarction-aetiology and prevention. Br J Anaesth 2005; 95:3-19.
38 Tsimikas S, Willerson JT, Ridker PM. C-reactive protein and other emerging blood biomarkers to optimize risk stratification of vulnerable patients. J Am Coll Cardiol 2006; 47 (8 Suppl):C19-C31.

39 Wang TJ, Larson MG, Levy D, Benjamin EJ, Leip EP, Omland T, Wolf PA, Vasan RS. Plasma natriuretic peptide levels and the risk of cardiovascular events and death. N Engl J Med 2004; 350:655-663.

40 Omland T, Sabatine MS, Jablonski KA, Rice MM, Hsia J, Wergeland R, Landaas S, Rouleau JL, Domanski MJ, Hall C, Pfeffer MA, Braunwald E. Prognostic value of B-type natriuretic peptides in patients with stable coronary artery disease: the PEACE Trial. J Am Coll Cardiol 2007; 50:205-214

41 Bibbins-Domingo K, Gupta R, Na B, Wu AHB, Schiller NB, Whooley MA. $\mathrm{N}$-Terminal fragment of the prohormone brain-type natriuretic peptide (NT-proBNP), cardiovascular events, and mortality in patients with stable coronary heart disease. JAMA 2007; 297:169-176.

42 Feringa $\mathrm{HHH}$, Bax JJ, Elhendy A, de Jonge R, Lindemans J, Schouten O, van den Meiracker AH, Boersma E, Schinkel AFL, Kertai MD. Association of plasma $\mathrm{N}$-terminal pro-B-type natriuretic peptide with postoperative cardiac events in patients undergoing surgery for abdominal aortic aneurysm or leg bypass. Am J Cardiol 2006; 98:111115.

43 Dernellis J, Panaretou M. Assessment of cardiac risk before non-cardiac surgery: brain natriuretic peptide in 1590 patients. Heart 2006; 92:1645-1650.

44 Rodseth RN, Padayachee L, Biccard BM. A meta-analysis of the utility of preoperative brain natriuretic peptide in predicting early and intermediateterm mortality and major adverse cardiac events in vascular surgical patients. Anaesthesia 2008; 63:1226-1233.

45 Cuthbertson BH, Card G, Croal BL, McNeilly J, Hillis GS. The utility of B-type natriuretic peptide in predicting postoperative cardiac events and mortality in patients undergoing major emergency non-cardiac surgery. Anaesthesia 2007; 62:875-881.

46 Cuthbertson BH, Amiri AR, Croal BL, Rajagopalan S, Alozairi O, Brittenden J, Hillis GS. Utility of B-type natriuretic peptide in predicting perioperative cardiac events in patients undergoing major non-cardiac surgery. Br J Anaesth 2007; 99:170-176.

47 Fox K, Garcia MA, Ardissino D, Buszman P, Camici PG, Crea F, Daly C, De Backer G, Hjemdahl P, Lopez-Sendon J, Marco J, Morais J, Pepper J, Sechtem U, Simoons M, Thygesen K. Guidelines on the management of stable angina pectoris: executive summary: the Task Force on the Management of Stable Angina Pectoris of the European Society of Cardiology. Eur Heart J 2006; 27:1341-1381.

48 Jeger RV, Probst C, Arsenic R, Lippuner T, Pfisterer ME, Seeberger MD, Filipovic M. Long-term prognostic value of the preoperative 12-lead electrocardiogram before major noncardiac surgery in coronary artery disease. Am Heart J 2006; 151:508-513.

49 Noordzij PG, Boersma E, Bax JJ, Feringa HH, Schreiner F, Schouten O, Kertai MD, Klein J, van Urk H, Elhendy A, Poldermans D. Prognostic value of routine preoperative electrocardiography in patients undergoing noncardiac surgery. Am J Cardiol 2006; 97:1103-1106.

50 Klocke FJ, Baird MG, Lorell BH, Bateman TM, Messer JV, Berman DS, O'Gara PT, Carabello BA, Russell RO Jr, Cerqueira MD, St John Sutton MG, DeMaria AN, Udelson JE, Kennedy JW, Verani MS, Williams KA, Antman EM, Smith SC Jr, Alpert JS, Gregoratos G, Anderson JL, Hiratzka LF, Faxon DP, Hunt SA, Fuster V, Jacobs AK, Gibbons RJ, Russell RO. ACC/AHA/ASNC guidelines for the clinical use of cardiac radionuclide imaging-executive summary: a report of the American College of Cardiology/American Heart Association Task Force on Practice Guidelines (ACC/AHA/ASNC Committee to Revise the 1995 Guidelines for the Clinical Use of Cardiac Radionuclide Imaging). J Am Coll Cardiol 2003; 42:1318-1333.

51 Kertai MD, Boersma E, Bax JJ, Heijenbrok-Kal MH, Hunink MG, L'Talien GJ, Roelandt JR, van Urk H, Poldermans D. A meta-analysis comparing the prognostic accuracy of six diagnostic tests for predicting perioperative cardiac risk in patients undergoing major vascular surgery. Heart 2003; 89:1327-1334.

52 Etchells E, Meade M, Tomlinson G, Cook D. Semiquantitative dipyridamole myocardial stress perfusion imaging for cardiac risk assessment before noncardiac vascular surgery: a metaanalysis. $J$ Vasc Surg 2002; 36:534-540.

53 Shaw LJ, Eagle KA, Gersh BJ, Douglas Miller D. Meta-analysis of intravenous dipyridamole-thallium-201 imaging (1985 to 1994) and dobutamine echocardiography ( 1991 to 1994) for risk stratification before vascular surgery. J Am Coll Cardiol 1996; 27:787-798.

54 Elhendy A, Valkema R, van Domburg RT, Bax JJ, Nierop PR, Cornel JH, Geleijnse ML, Reijs AE, Krenning EP, Roelandt JR. Safety of dobutamineatropine stress myocardial perfusion scintigraphy. J Nucl Med 1998; 39:1662-1666. 
55 Sicari R, Nihoyannopoulos P, Evangelista A, Kasprzak J, Lancellotti P, Poldermans D, Voigt JU, Zamorano JL. Stress Echocardiography Expert Consensus Statement-Executive Summary: European Association of Echocardiography (EAE) (a registered branch of the ESC). Eur Heart J 2009; 30:278-289.

56 Das MK, Pellikka PA, Mahoney DW, Roger VL, Oh JK, McCully RB, Seward JB. Assessment of cardiac risk before nonvascular surgery: dobutamine stress echocardiography in 530 patients. J Am Coll Cardiol 2000; 35:1647-1653.

57 Nandalur KR, Dwamena BA, Choudhri AF, Nandalur MR, Carlos RC. Diagnostic performance of stress cardiac magnetic resonance imaging in the detection of coronary artery disease: a meta-analysis. J Am Coll Cardiol 2007; 50:1343-1353.

58 Rerkpattanapipat P, Morgan TM, Neagle CM, Link KM, Hamilton CA, Hundley WG. Assessment of preoperative cardiac risk with magnetic resonance imaging. A mJ Cardiol 2002; 90:416-419.

59 Danias PG, Roussakis A, loannidis JP. Diagnostic performance of coronary magnetic resonance angiography as compared against conventional X-ray angiography: a meta-analysis. J Am Coll Cardio/ 2004; 44:1867-1876.

60 Hamon M, Biondi-Zoccai GG, Malagutti P, Agostoni P, Morello R, Valgimigli M, Hamon M. Diagnostic performance of multislice spiral computed tomography of coronary arteries as compared with conventional invasive coronary angiography: a meta-analysis. J Am Coll Cardiol 2006; 48:1896-1910.

61 Gerber TC, Carr JJ, Arai AE, Dixon RL, Ferrari VA, Gomes AS, Heller GV, McCollough CH, McNitt-Gray MF, Mettler FA, Mieres JH, Morin RL, Yester MV. lonizing radiation in cardiac imaging: a science advisory from the American Heart Association Committee on Cardiac Imaging of the Council on Clinical Cardiology and Committee on Cardiovascular Imaging and Intervention of the Council on Cardiovascular Radiology and Intervention. Circulation 2009; 119:1056-1065.

62 Pouleur AC, le Polain de Waroux JB, Kefer J, Pasquet A, Coche E, Vanoverschelde JL, Gerber BL. Usefulness of 40-slice multidetector row computed tomography to detect coronary disease in patients prior to cardiac valve surgery. Eur Radiol 2007; 17:3199-3207.

63 Boersma E, Poldermans D, Bax JJ, Steyerberg EW, Thomson IR, Banga JD, van De Ven LL, van Urk H, Roelandt JR. Predictors of cardiac events after major vascular surgery: role of clinical characteristics, dobutamine echocardiography, and beta-blocker therapy. JAMA 2001; 285:18651873.

64 ATS/ACCP Statement on Cardiopulmonary Exercise Testing. Am J Respir Crit Care Med 2003;167:211-277.

65 Reilly CS. Can we accurately assess an individual's perioperative risk? $\mathrm{Br}$ J Anaesth 2008; 101:747-749.

66 Brunelli A, Belardinelli R, Refai M, Salati M, Socci L, Pompili C, Sabbatini A. Peak oxygen consumption during cardiopulmonary exercise test improves risk stratification in candidates to major lung resection. Chest 2009; 135:1260-1267.

67 Older P, Smith R, Courtney P, Hone R. Preoperative evaluation of cardiac failure and ischemia in elderly patients by cardiopulmonary exercise testing. Chest 1993; 104:701-704.

68 Piepoli MF, Corra U, Agostoni PG, Belardinelli R, Cohen-Solal A, Hambrecht R, Vanhees L. Statement on cardiopulmonary exercise testing in chronic heart failure due to left ventricular dysfunction.

Recommendations for performance and interpretation. Eur J Cardiovasc Prev Rehabil 2006; 13:10-12.

69 Bassand JP, Hamm CW, Ardissino D, Boersma E, Budaj A, FernandezAviles F, Fox KA, Hasdai D, Ohman EM, Wallentin L, Wijns W. Guidelines for the diagnosis and treatment of non-ST-segment elevation acute coronary syndromes. Eur Heart J 2007; 28:1598-1660.

70 Silber S, Albertsson P, Aviles FF, Camici PG, Colombo A, Hamm C, Jorgensen E, Marco J, Nordrehaug JE, Ruzyllo W, Urban P, Stone GW, Wijns W, Guidelines for percutaneous coronary interventions. The Task Force for Percutaneous Coronary Interventions of the European Society of Cardiology. Eur Heart J 2005; 26:804-847.

71 Van de Werf F, Bax J, Betriu A, Blomstrom-Lundqvist C, Crea F, Falk V, Filippatos G, Fox K, Huber K, Kastrati A, Rosengren A, Steg PG, Tubaro $M$, Verheugt $F$, Weidinger $F$, Weis M. Management of acute myocardial infarction in patients presenting with persistent ST-segment elevation: the Task Force on the management of ST-segment elevation acute myocardial infarction of the European Society of Cardiology. Eur Heart J 2008; 29:2909-2945.

72 Cruickshank JM. Are we misunderstanding beta-blockers. Int J Cardiol 2007; 120:10-27.

73 Raby KE, Brull SJ, Timimi F, Akhtar S, Rosenbaum S, Naimi C, Whittemore AD. The effect of heart rate control on myocardial ischemia among highrisk patients after vascular surgery. Anesth Analg 1999; 88:477-482.
74 Brady AR, Gibbs JS, Greenhalgh RM, Powell JT, Sydes MR. Perioperative beta-blockade (POBBLE) for patients undergoing infrarenal vascular surgery: results of a randomized double-blind controlled trial. J Vasc Surg 2005; 41:602-609.

75 Juul AB, Wetterslev J, Gluud C, Kofoed-Enevoldsen A, Jensen G, Callesen T, Norgaard P, Fruergaard K, Bestle M, Vedelsdal R, Miran A, Jacobsen J, Roed J, Mortensen MB, Jorgensen L, Jorgensen J, Rovsing ML, Petersen PL, Pott F, Haas M, Albret R, Nielsen LL, Johansson G, Stjernholm P, Molgaard Y, Foss NB, Elkjaer J, Dehlie B, Boysen K, Zaric D, Munksgaard A, Madsen JB, Oberg B, Khanykin B, Blemmer T, Yndgaard S, Perko G, Wang LP, Winkel P, Hilden J, Jensen P, Salas N. Effect of perioperative beta blockade in patients with diabetes undergoing major non-cardiac surgery: randomised placebo controlled, blinded multicentre trial. BMJ 2006; 332:1482.

76 Mangano DT, Layug EL, Wallace A, Tateo I. Effect of atenolol on mortality and cardiovascular morbidity after noncardiac surgery. Multicenter Study of Perioperative Ischemia Research Group. N Engl J Med 1996; 335:1713-1720.

77 Yang H, Raymer K, Butler R, Parlow J, Roberts R. The effects of perioperative beta-blockade: results of the Metoprolol after Vascular Surgery (MaVS) study, a randomized controlled trial. Am Heart J 2006; 152:983-990.

78 Zaugg M, Bestmann L, Wacker J, Lucchinetti E, Boltres A, Schulz C, Hersberger M, Kalin G, Furrer L, Hofer C, Blumenthal S, Muller A, Zollinger A, Spahn DR, Borgeat A. Adrenergic receptor genotype but not peri-operative bisoprolol therapy may determine cardiovascular outcome in at-risk patients undergoing surgery with spinal block: the Swiss Beta Blocker in Spinal Anesthesia (BBSA) study: a double-blinded, placebocontrolled, multicenter trial with 1-year follow-up. Anesthesiology 2007; 107:33-44.

79 Auerbach AD, Goldman L. $\beta$-Blockers and reduction of cardiac events in noncardiac surgery: scientific review. JAMA 2002; 287:14351444.

80 McGory ML, Maggard MA, Ko CY. A meta-analysis of perioperative beta blockade: what is the actual risk reduction? Surgery 2005; 138:171179.

81 Schouten O, Shaw LJ, Boersma E, Bax JJ, Kertai MD, Feringa HH, Biagini E, Kok NF, Urk H, Elhendy A, Poldermans D. A meta-analysis of safety and effectiveness of perioperative beta-blocker use for the prevention of cardiac events in different types of noncardiac surgery. Coron Artery Dis 2006; 17:173-179.

82 Stevens RD, Burri H, Tramer MR. Pharmacologic myocardial protection in patients undergoing noncardiac surgery: a quantitative systematic review. Anesth Analg 2003; 97:623-633.

83 Bangalore S, Wetterslev J, Pranesh S, Sahney S, Gluud C, Messerli FH. Perioperative $\beta$ blockers in patients having non-cardiac surgery: a metaanalysis. Lancet 2008; 372:1962-1976.

84 Devereaux PJ, Beattie WS, Choi PT, Badner NH, Guyatt GH, Villar JC, Cina CS, Leslie K, Jacka MJ, Montori VM, Bhandari M, Avezum A, Cavalcanti AB, Giles JW, Schricker T, Yang H, Jakobsen CJ, Yusuf S. How strong is the evidence for the use of perioperative beta blockers in non-cardiac surgery? Systematic review and meta-analysis of randomised controlled trials. BMJ 2005; 331:313-321.

85 Wiesbauer F, Schlager O, Domanovits H, Wildner B, Maurer G, Muellner $M$, Blessberger $H$, Schillinger M. Perioperative beta-blockers for preventing surgery-related mortality and morbidity: a systematic review and meta-analysis. Anesth Analg 2007; 104:27-41.

86 Beattie WS, Wijeysundera DN, Karkouti K, McCluskey S, Tait G. Does tight heart rate control improve beta-blocker efficacy? An updated analysis of the noncardiac surgical randomized trials. Anesth Analg 2008; 106:1039-1048.

87 Lindenauer PK, Pekow P, Wang K, Mamidi DK, Gutierrez B, Benjamin EM. Peri-operative beta-blocker therapy and mortality after major noncardiac surgery. N Engl J Med 2005; 353:349-361.

88 Dunkelgrun M, Boersma E, Schouten O, Koopman-Van Gemert AWMM, Van Poorten F, Bax JJ, Thomson IR, Poldermans D. Bisoprolol and fluvastin for the reduction of perioperative cardiac mortality and myocardial infarction in intermediate-risk patients undergoing noncardiovascular surgery; a randomized controlled trial (DECREASE-IV). Ann Surg 2009; 249:921-926.

89 Hoeks SE, Scholte Op Reimer WJ, van Urk H, Jorning PJ, Boersma E, Simoons ML, Bax JJ, Poldermans D. Increase of 1-year mortality after perioperative beta-blocker withdrawal in endovascular and vascular surgery patients. Eur J Vasc Endovasc Surg 2007; 33:13-19.

90 Shammash JB, Trost JC, Gold JM, Berlin JA, Golden MA, Kimmel SE. Perioperative beta-blocker withdrawal and mortality in vascular surgical patients. Am Heart J 2001; 41:148-153. 
91 Dickstein K, Cohen-Solal A, Filippatos G, McMurray JJ, Ponikowski P, Poole-Wilson PA, Stromberg A, van Veldhuisen DJ, Atar D, Hoes AW, Keren A, Mebazaa A, Nieminen M, Priori SG, Swedberg K. ESC Guidelines for the diagnosis and treatment of acute and chronic heart failure 2008: The Task Force for the Diagnosis and Treatment of Acute and Chronic Heart Failure 2008 of the European Society of Cardiology. Developed in collaboration with the Heart Failure Association of the ESC (HFA) and endorsed by the European Society of Intensive Care Medicine (ESICM). Eur Heart J 2008; 29:2388-2442.

92 Paravastu SC, Mendonca D, Da Silva A. Beta blockers for peripheral arterial disease. Cochrane Database Syst Rev 2008;(4):CD005508.

93 van Gestel YRBM, Hoeks SE, Sin DD, Welten GMJM, Schouten O, Witteveen HJ, Simsek C, Stam H, Mertens FW, Bax JJ, van Domburg RT, Poldermans D. Impact of cardioselective $\beta$-blockers on mortality in patients with chronic obstructive pulmonary disease and atherosclerosis. Am J Respir Crit Care Med 2008; 178:695-700.

94 Feringa HHH, Bax JJ, Boersma E, Kertai MD, Meij SH, Galal W, Schouten O, Thomson IR, Klootwijk P, van Sambeek MRHM, Klein J, Poldermans D. High-dose beta-blockers and tight heart rate control reduce myocardialischemia and troponin $\mathrm{T}$ release in vascular surgery patients. Circulation 2006; 114 (1 Suppl):|344-I349.

95 Boersma E, Poldermans D. Beta blockers in non-cardiac surgery: haemodynamic data needed. Lancet 2008; 372:1930-1932.

96 Graham I, Atar D, Borch-Johnsen K, Boysen G, Burell G, Cifkova R, Dallongeville J, De Backer G, Ebrahim S, Gjelsvik B, Herrmann-Lingen C, Hoes A, Humphries S, Knapton M, Perk J, Priori SG, Pyorala K, Reiner Z, Ruilope L, Sans-Menendez S, Scholte op Reimer W, Weissberg P, Wood $\mathrm{D}$, Yarnell J, Zamorano JL. European guidelines on cardiovascular disease prevention in clinical practice: executive summary. Eur Heart J 2007; 28:2375-2414.

97 Rosenson RS, Tangney CC. Antiatherothrombotic properties of statins: implications for cardiovascular event reduction. JAMA 1998; 279:16431650.

98 Hindler K, Shaw AD, Samuels J, Fulton S, Collard CD, Riedel B. Improved postoperative outcomes associated with preoperative statin therapy. Anesthesiology 2006; 105:1260-1272; quiz 1289-1290.

99 Williams TM, Harken AH. Statins for surgical patients. Ann Surg 2008; 247:30-37.

100 Durazzo AE, Machado FS, Ikeoka DT, De Bernoche C, Monachini MC, Puech-Leao P, Caramelli B. Reduction in cardiovascular events after vascular surgery with atorvastatin: a randomized trial. J Vasc Surg 2004; 39:967-975.

101 Schouten O, Boersma E, Hoeks S, Benner R, van Urk H, van Sambeek MRHM, Verhagen HJM, Khan NA, Dunkelgrun M, Bax JJ, Poldermans D. Fluvastatin $\mathrm{XL}$ use is associated with improved cardiac outcome after major vascular surgery. Results from a randomized placebo controlled trial: DECREASE III. Eur Heart J 2008; 29(abstract supplement)(Hotline session ESC).

102 Schouten O, Kertai MD, Bax JJ, Durazzo AE, Biagini E, Boersma E, van Waning VH, Lameris TW, van Sambeek MR, Poldermans D. Safety of perioperative statin use in high-risk patients undergoing major vascular surgery. Am J Cardiol 2005; 95:658-660.

103 Schouten O, Hoeks SE, Welten GM, Davignon J, Kastelein JJ, Vidakovic $\mathrm{R}$, Feringa HH, Dunkelgrun M, van Domburg RT, Bax JJ, Poldermans D. Effect of statin withdrawal on frequency of cardiac events after vascular surgery. Am J Cardiol 2007; 100:316-320.

104 Coriat P, Daloz M, Bousseau D, Fusciardi J, Echter E, Viars P. Prevention of intraoperative myocardial ischemia during noncardiac surgery with intravenous nitroglycerin. Anesthesiology 1984; 61:193-196.

105 Dodds TM, Stone JG, Coromilas J, Weinberger M, Levy DG. Prophylactic nitroglycerin infusion during noncardiac surgery does not reduce perioperative ischemia. Anesth Analg 1993; 76:705-713.

106 Sun YP, Zhu BQ, Browne AE, Pulukurthy S, Chou TM, Sudhir K, Glantz SA, Deedwania PC, Chatterjee K, Parmley WW. Comparative effects of ACE inhibitors and an angiotensin receptor blocker on atherosclerosis and vascular function. J Cardiovasc Pharmacol Ther 2001; 6:175-181.

107 Oosterga M, Voors AA, Pinto YM, Buikema H, Grandjean JG, Kingma JH, Crijns $\mathrm{HJ}$, van Gilst WH. Effects of quinapril on clinical outcome after coronary artery bypass grafting (The QUO VADIS Study). QUinapril on Vascular Ace and Determinants of Ischemia. Am J Cardiol 2001; 87:542-546.

108 Filion KB, Pilote L, Rahme E, Eisenberg MJ. Perioperative use of cardiac medical therapy among patients undergoing coronary artery bypass graft surgery: a systematic review. Am Heart J 2007; 154:407-414.

109 Wijeysundera DN, Beattie WS. Calcium channel blockers for reducing cardiac morbidity after noncardiac surgery: a meta-analysis. Anesth Analg 2003; 97:634-641.
110 Kertai MD, Westerhout CM, Varga KS, Acsady G, Gal J. Dihydropiridine calcium-channel blockers and perioperative mortality in aortic aneurysm surgery. $\mathrm{Br} J$ Anaesth 2008; 101:458-465.

111 Shchukin lu V, Vachev AN, Surkova EA, Germanov AV, Golovin EA, D'lachkov VA. The role of beta-adrenoblockers and If-inhibitor ivabradine in lowering of rate of development of cardiac complications after carotid endarterectomy. Kardiologiia 2008; 48:56-59.

112 Oliver MF, Goldman L, Julian DG, Holme I. Effect of mivazerol on perioperative cardiac complications during non-cardiac surgery in patients with coronary heart disease: the European Mivazerol Trial (EMIT). Anesthesiology 1999; 91:951-961.

113 Wallace AW, Galindez D, Salahieh A, Layug EL, Lazo EA, Haratonik KA, Boisvert DM, Kardatzke D. Effect of clonidine on cardiovascular morbidity and mortality after noncardiac surgery. Anesthesiology 2004; 101:284293.

114 Wijeysundera DN, Naik JS, Beattie WS. Alpha-2 adrenergic agonists to prevent perioperative cardiovascular complications: a meta-analysis. $\mathrm{Am} J$ Med 2003; 114:742-752.

115 Vitez TS, Soper LE, Wong KC, Soper P. Chronic hypokalemia and intraoperative dysrhythmias. Anesthesiology 1985; 63:130-133.

116 Macdonald JE, Struthers AD. What is the optimal serum potassium level in cardiovascular patients? J Am Coll Cardiol 2004; 43:155-161.

117 Shah KB, Kleinman BS, Rao TL, Jacobs HK, Mestan K, Schaafsma M. Angina and other risk factors in patients with cardiac diseases undergoing noncardiac operations. Anesth Analg 1990; 70:240-247.

118 Domanski M, Norman J, Pitt B, Haigney M, Hanlon S, Peyster E. Diuretic use, progressive heart failure, and death in patients in the Studies Of Left Ventricular Dysfunction (SOLVD). J Am Coll Cardiol 2003; 42:705-708.

119 Lindblad B, Persson NH, Takolander R, Bergqvist D. Does low-dose acetylsalicylic acid prevent stroke after carotid surgery? A double-blind, placebo-controlled randomized trial. Stroke 1993; 24:1125-1128.

120 Robless P, Mikhailidis DP, Stansby G. Systematic review of antiplatelet therapy for the prevention of myocardial infarction, stroke or vascular death in patients with peripheral vascular disease. Br J Surg 2001; 88:787-800.

121 Burger W, Chemnitius JM, KneissI GD, Rucker G. Low-dose aspirin for secondary cardiovascular prevention-cardiovascular risks after its perioperative withdrawal versus bleeding risks with its continuationreview and meta-analysis. J Intern Med 2005; 257:399-414.

122 Biondi-Zoccai GG, Lotrionte M, Agostoni P, Abbate A, Fusaro M, Burzotta F, Testa L, Sheiban I, Sangiorgi G. A systematic review and meta-analysis on the hazards of discontinuing or not adhering to aspirin among 50,279 patients at risk for coronary artery disease. Eur Heart J 2006; 27:26672674.

123 Douketis JD, Johnson JA, Turpie AG. Low-molecular-weight heparin as bridging anticoagulation during interruption of warfarin: assessment of a standardized periprocedural anticoagulation regimen. Arch Intern Med 2004; 164:1319-1326.

124 Vahanian A, Baumgartner H, Bax J, Butchart E, Dion R, Filippatos G, Flachskampf F, Hall R, lung B, Kasprzak J, Nataf P, Tornos P, Torracca L, Wenink A. Guidelines on the management of valvular heart disease: the Task Force on the Management of Valvular Heart Disease of the European Society of Cardiology. Eur Heart J 2007; 28:230-268.

125 De Caterina R, Husted S, Wallentin L, Agnelli G, Bachmann F, Baigent C, Jespersen J, Kristensen SD, Montalescot G, Siegbahn A, Verheugt FW, Weitz J. Anticoagulants in heart disease: current status and perspectives. Eur Heart J 2007; 28:880-913.

126 Pengo V, Cucchini U, Denas G, Erba N, Guazzaloca G, La Rosa L, De Micheli V, Testa S, Frontoni R, Prisco D, Nante G, lliceto S, for the Italian Federation of Centers for the Diagnosis of Thrombosis and Management of Antithrombotic T. Standardized low-molecular-weight heparin bridging regimen in outpatients on oral anticoagulants undergoing invasive procedure or surgery. an inception cohort management study. Circulation 2009; 119:2920-2927.

127 Poldermans D, Boersma E, Bax JJ, Kliffen M, van Urk H, van de Ven L, Roelandt JR, Thomson IR. Correlation of location of acute myocardial infarct after noncardiac vascular surgery with preoperative dobutamine echocardiographic findings. AmJ Cardiol 2001; 88:1413-1414.

128 Eagle KA, Rihal CS, Mickel MC, Holmes DR, Foster ED, Gersh BJ. Cardiac risk of noncardiac surgery: influence of coronary disease and type of surgery in 3368 operations. CASS Investigators and University of Michigan Heart Care Program. Coronary Artery Surgery Study. Circulation 1997; 96:1882-1887.

129 Huber KC, Evans MA, Bresnahan JF, Gibbons RJ, Holmes DR Jr. Outcome of noncardiac operations in patients with severe coronary artery disease successfully treated preoperatively with coronary angioplasty. Mayo Clin Proc 1992; 67:15-21. 
130 Kaluza GL, Joseph J, Lee JR, Raizner ME, Raizner AE. Catastrophic outcomes of noncardiac surgery soon after coronary stenting. J Am Coll Cardiol 2000; 35:1288-1294.

131 Howard-Alpe GM, de Bono J, Hudsmith L, Orr WP, Foex P, Sear JW. Coronary artery stents and non-cardiac surgery. $\mathrm{Br} J$ Anaesth 2007; 98:560-574.

132 Nuttall GA, Brown MJ, Stombaugh JW, Michon PB, Hathaway MF, Lindeen KC, Hanson AC, Schroeder DR, Oliver WC, Holmes DR, Rihal $\mathrm{CS}$. Time and cardiac risk of surgery after bare-metal stent percutaneous coronary intervention. Anesthesiology 2008; 109:588-595.

133 Rabbitts JA, Nuttall GA, Brown MJ, Hanson AC, Oliver WC, Holmes DR Rihal CS. Cardiac risk of noncardiac surgery after percutaneous coronary intervention with drug-eluting stents. Anesthesiology 2008; 109:596604.

134 Douketis JD, Berger PB, Dunn AS, Jaffer AK, Spyropoulos AC Becker RC, Ansell J. The perioperative management of antithrombotic therapy: American College of Chest Physicians Evidence-Based Clinical Practice Guidelines (8th Edition). Chest 2008; 133 (6 Suppl):299S339S.

135 McFalls EO, Ward HB, Moritz TE, Goldman S, Krupski WC, Littooy F, Pierpont G, Santilli S, Rapp J, Hattler B, Shunk K, Jaenicke C, Thottapurathu L, Ellis N, Reda DJ, Henderson WG. Coronary-artery revascularization before elective major vascular surgery. $N$ Engl J Med 2004; 351:2795-2804.

136 Poldermans D, Schouten O, Vidakovic R, Bax JJ, Thomson IR, Hoeks SE, Feringa $\mathrm{HH}$, Dunkelgrun $\mathrm{M}$, de Jaegere $\mathrm{P}$, Maat $\mathrm{A}$, van Sambeek MR, Kertai MD, Boersma E. A clinical randomized trial to evaluate the safety of a noninvasive approach in high-risk patients undergoing major vascular surgery: the DECREASE-V Pilot Study. J Am Coll Cardiol 2007; 49:1763-1769.

137 Serruys PW, Morice MC, Kappetein AP, Colombo A, Holmes DR, Mack MJ, Stahle E, Feldman TE, van den Brand M, Bass EJ, Van Dyck N, Leadley K, Dawkins KD, Mohr FW. Percutaneous coronary intervention versus coronary-artery bypass grafting for severe coronary artery disease. N Engl J Med 2009; 360:961-972.

138 Kazmers A, Cerqueira MD, Zierler RE. Perioperative and late outcome in patients with left ventricular ejection fraction of $35 \%$ or less who require major vascular surgery. J Vasc Surg 1988; 8:307-315.

139 Hammill BG, Curtis LH, Bennett-Guerrero E, O'Connor CM, Jollis JG, Schulman KA, Hernandez AF. Impact of heart failure on patients undergoing major noncardiac surgery. Anesthesiology 2008; 108:559567.

140 Bhatia RS, Tu JV, Lee DS, Austin PC, Fang J, Haouzi A, Gong Y, Liu PP. Outcome of heart failure with preserved ejection fraction in a populationbased study. N Engl J Med 2006; 355:260-269.

141 Karagiannis SE, Feringa HH, Vidakovic R, van Domburg R, Schouten O, Bax JJ, Karatasakis G, Cokkinos DV, Poldermans D. Value of myocardial viability estimation using dobutamine stress echocardiography in assessing risk preoperatively before noncardiac vascular surgery in patients with left ventricular ejection fraction, 35\%. Am J Cardio/ 2007; 99:1555-1559.

142 Feringa $\mathrm{HH}$, Bax JJ, Schouten O, Poldermans D. Protecting the heart with cardiac medication in patients with left ventricular dysfunction undergoing major noncardiac vascular surgery. Semin Cardiothorac Vasc Anesth 2006; 10:25-31.

143 Mancia G, De Backer G, Dominiczak A, Cifkova R, Fagard R, Germano G, Grassi G, Heagerty AM, Kjeldsen SE, Laurent S, Narkiewicz K, Ruilope L, Rynkiewicz A, Schmieder RE, Struijker Boudier HA, Zanchetti A. the Task Force for the management of arterial hypertension of the European Society of Hypertension, the Task Force for the management of arterial hypertension of the European Society of C. 2007 Guidelines for the management of arterial hypertension: The Task Force for the Management of Arterial Hypertension of the European Society of Hypertension (ESH) and of the European Society of Cardiology (ESC). Eur Heart J 2007; 28:1462-1536.

144 Weksler N, Klein M, Szendro G, Rozentsveig V, Schily M, Brill S, Tarnopolski A, Ovadia L, Gurman GM. The dilemma of immediate preoperative hypertension: to treat and operate, or to postpone surgery? J Clin Anesth 2003; 15:179-183.

145 lung B, Baron G, Butchart EG, Delahaye F, Gohlke-Barwolf C, Levang OW, Tornos P, Vanoverschelde JL, Vermeer F, Boersma E, Ravaud P, Vahanian A. A prospective survey of patients with valvular heart disease in Europe: the Euro Heart Survey on Valvular Heart Disease. Eur Heart J 2003; 24:1231-1243.

146 Kertai MD, Bountioukos M, Boersma E, Bax JJ, Thomson IR, Sozzi F, Klein J, Roelandt JRTC, Poldermans D. Aortic stenosis: an underestimated risk factor for perioperative complications in patients undergoing noncardiac surgery. Am J Med 2004; 116:8-13.
147 Vahanian A, Alfieri O, Al-Attar N, Antunes M, Bax J, Cormier B, Cribier A, De Jaegere $P$, Fournial G, Kappetein AP, Kovac J, Ludgate S, Maisano F, Moat N, Mohr F, Nataf P, Pierard L, Pomar JL, Schofer J, Tornos P, Tuzcu M, van Hout B, Von Segesser LK, Walther T. Transcatheter valve implantation for patients with aortic stenosis: a position statement from the European Association of Cardio-Thoracic Surgery (EACTS) and the European Society of Cardiology (ESC), in collaboration with the European Association of Percutaneous Cardiovascular Interventions (EAPCl). Eur Heart J 2008; 29:1463-1470.

148 Habib G, Hoen B, Tornos P, Thuny F, Prendergast B, Vilacosta I, Moreillon $P$, de Jesus Antunes M, Thilen U, Lekakis J, Lengyel M, Mueller L, Naber CK, Nihoyannopoulos P, Moritz A, Zamorano GL. Guidelines on the prevention, diagnosis, and treatment of infective endocarditis (new version 2009). Eur Heart J; doi:10.1093/eurheartj/ehp285.

149 Wilson W, Taubert KA, Gewitz M, Lockhart PB, Baddour LM, Levison M, Bolger A, Cabell CH, Takahashi M, Baltimore RS, Newburger JW, Strom BL, Tani LY, Gerber M, Bonow RO, Pallasch T, Shulman ST, Rowley AH, Burns JC, Ferrieri P, Gardner T, Goff D, Durack DT. Prevention of infective endocarditis: guidelines from the American Heart Association: a guideline from the American Heart Association Rheumatic Fever, Endocarditis, and Kawasaki Disease Committee, Council on Cardiovascular Disease in the Young, and the Council on Clinical Cardiology, Council on Cardiovascular Surgery and Anesthesia, and the Quality of Care and Outcomes Research Interdisciplinary Working Group. Circulation 2007; 116:1736-1754.

150 Forrest JB, Cahalan MK, Rehder K, Goldsmith CH, Levy WJ, Strunin L, Bota W, Boucek CD, Cucchiara RF, Dhamee S, Domino KB, Dudman AJ, Hamilton WK, Kampine J, Kotrly KJ, Maltby JR, Mazloomdoost M, Mackenzie RA, Melnick BM, Motoyama E, Muir JJ, Munshi C. Multicenter study of general anesthesia. II. Results. Anesthesiology 1990; 72:262268.

151 Forrest JB, Rehder K, Cahalan MK, Goldsmith CH. Multicenter study of general anesthesia. III. Predictors of severe perioperative adverse outcomes. Anesthesiology 1992; 76:3-15.

152 Katz RL, Bigger JT Jr. Cardiac arrhythmias during anesthesia and operation. Anesthesiology 1970; 33:193-213.

153 Bertrand CA, Steiner NV, Jameson AG, Lopez M. Disturbances of cardiac rhythm during anesthesia and surgery. JAMA 1971; 216:16151617.

154 Zipes DP, Camm AJ, Borggrefe M, Buxton AE, Chaitman B, Fromer M, Gregoratos G, Klein G, Moss AJ, Myerburg RJ, Priori SG, Quinones MA, Roden DM, Silka MJ, Tracy C, Smith SC Jr, Jacobs AK, Adams CD, Antman EM, Anderson JL, Hunt SA, Halperin JL, Nishimura R, Ornato JP, Page RL, Riegel B, Blanc JJ, Budaj A, Camm AJ, Dean V, Deckers JW, Despres C, Dickstein K, Lekakis J, McGregor K, Metra M, Morais J, Osterspey A, Tamargo JL, Zamorano JL. ACC/AHA/ESC 2006 guidelines for management of patients with ventricular arrhythmias and the prevention of sudden cardiac death: a report of the American College of Cardiology/American Heart Association Task Force and the European Society of Cardiology Committee for Practice Guidelines (Writing Committee to Develop Guidelines for Management of Patients with Ventricular Arrhythmias and the Prevention of Sudden Cardiac Death). $J$ Am Coll Cardiol 2006; 48:e247-e346.

155 Amar D. Perioperative atrial tachyarrhythmias. Anesthesiology 2002; 97:1618-1623.

156 Cox JL. A perspective of postoperative atrial fibrillation in cardiac operations. Ann Thorac Surg 1993; 56:405-409.

157 O'Kelly B, Browner WS, Massie B, Tubau J, Ngo L, Mangano DT. Ventricular arrhythmias in patients undergoing noncardiac surgery. The Study of Perioperative Ischemia Research Group. JAMA 1992; 268:217-221.

158 Polanczyk CA, Goldman L, Marcantonio ER, Orav EJ, Lee TH. Supraventricular arrhythmia in patients having noncardiac surgery: clinical correlates and effect on length of stay. Ann Intern Med 1998; 129:279285.

159 Amar D, Zhang H, Miodownik S, Kadish AH. Competing autonomic mechanisms precede the onset of postoperative atrial fibrillation. $J$ Am Coll Cardiol 2003; 42:1262-1268.

160 Atlee JL. Perioperative cardiac dysrhythmias: diagnosis and management. Anesthesiology 1997; 86:1397-1424.

161 Fuster V, Ryden LE, Cannom DS, Crijns HJ, Curtis AB, Ellenbogen KA Halperin JL, Le Heuzey JY, Kay GN, Lowe JE, Olsson SB, Prystowsky EN, Tamargo JL, Wann S. ACC/AHA/ESC 2006 guidelines for the management of patients with atrial fibrillation-executive summary: a report of the American College of Cardiology/American Heart Association Task Force on Practice Guidelines and the European Society of Cardiology Committee for Practice Guidelines (Writing Committee to Revise the 2001 Guidelines for the Management of Patients with Atrial Fibrillation). Eur Heart J 2006; 27:1979-2030. 
162 Maisel WH, Rawn JD, Stevenson WG. Atrial fibrillation after cardiac surgery. Ann Intern Med 2001; 135:1061-1073.

163 Balser JR. Perioperative arrhythmias: incidence, risk assessment, evaluation, and management. Card Electrophysiol Rev 2002; 6:96-99.

164 Balser JR, Martinez EA, Winters BD, Perdue PW, Clarke AW, Huang W, Tomaselli GF, Dorman T, Campbell K, Lipsett P, Breslow MJ, Rosenfeld BA. Beta-adrenergic blockade accelerates conversion of postoperative supraventricular tachyarrhythmias. Anesthesiology 1998; 89:10521059.

165 Bayliff CD, Massel DR, Inculet RI, Malthaner RA, Quinton SD, Powell FS, Kennedy RS. Propranolol for the prevention of postoperative arrhythmias in general thoracic surgery. Ann Thorac Surg 1999; 67:182 186.

166 Jakobsen CJ, Bille S, Ahlburg P, Rybro L, Hjortholm K, Andresen EB. Perioperative metoprolol reduces the frequency of atrial fibrillation after thoracotomy for lung resection. J Cardiothorac Vasc Anesth 1997; 11:746-751.

167 Mahla E, Rotman B, Rehak P, Atlee JL, Gombotz H, Berger J, List WF, Klein W, Metzler H. Perioperative ventricular dysrhythmias in patients with structural heart disease undergoing noncardiac surgery. Anesth Analg 1998; 86:16-21.

168 Vardas PE, Auricchio A, Blanc JJ, Daubert JC, Drexler H, Ector H, Gasparini M, Linde C, Morgado FB, Oto A, Sutton R, Trusz-Gluza M. Guidelines for cardiac pacing and cardiac resynchronization therapy: The Task Force for Cardiac Pacing and Cardiac Resynchronization Therapy of the European Society of Cardiology. Developed in collaboration with the European Heart Rhythm Association. Eur Heart J 2007; 28:2256-2295.

169 Eagle KA, Berger PB, Calkins H, Chaitman BR, Ewy GA, Fleischmann KE, Fleisher LA, Froehlich JB, Gusberg RJ, Leppo JA, Ryan T, Schlant RC, Winters WL Jr, Gibbons RJ, Antman EM, Alpert JS, Faxon DP, Fuster V, Gregoratos G, Jacobs AK, Hiratzka LF, Russell RO, Smith SC Jr. ACC/AHA guideline update for perioperative cardiovascular evaluation for noncardiac surgery-executive summary: a report of the American College of Cardiology/ American Heart Association Task Force on Practice Guidelines (Committee to Update the 1996 Guidelines on Perioperative Cardiovascular Evaluation for Noncardiac Surgery). J Am Coll Cardiol 2002; 39:542-553.

170 Gregoratos G, Cheitlin MD, Conill A, Epstein AE, Fellows C, Ferguson TB Jr, Freedman RA, Hlatky MA, Naccarelli GV, Saksena S, Schlant RC, Silka MJ, Ritchie JL, Gibbons RJ, Cheitlin MD, Eagle KA, Gardner TJ, Lewis RP, O'Rourke RA, Ryan TJ, Garson A Jr. ACC/AHA guidelines for implantation of cardiac pacemakers and antiarrhythmia devices: a report of the American College of Cardiology/American Heart Association Task Force on Practice Guidelines (Committee on Pacemaker Implantation). J Am Coll Cardiol 1998; 31:1175-1209.

171 Erdman S, Levinsky L, Servadio C, Stoupel E, Levy MJ. Safety precautions in the management of patients with pacemakers when electrocautery operations are performed. Surg Gynecol Obstet 1988; 167:311-314.

172 Potyk D, Raudaskoski P. Preoperative cardiac evaluation for elective noncardiac surgery. Arch Fam Med 1998; 7:164-173.

173 Simon AB. Perioperative management of the pacemaker patient. Anesthesiology 1977; 46:127-131.

174 Shapiro WA, Roizen MF, Singleton MA, Morady F, Bainton CR, Gaynor RL. Intraoperative pacemaker complications. Anesthesiology 1985; 63:319-322.

175 Madigan JD, Choudhri AF, Chen J, Spotnitz HM, Oz MC, Edwards N. Surgical management of the patient with an implanted cardiac device: implications of electromagnetic interference. Ann Surg 1999; 230:639647.

176 Pinski SL, Trohman RG. Implantable cardioverter-defibrillators: implications for the nonelectrophysiologist. Ann Intern Med 1995; 122:770-777.

177 Cockcroft DW, Gault MH. Prediction of creatinine clearance from serum creatinine. Nephron 1976; 16:31-41.

178 Kertai MD, Boersma E, Bax JJ, van den Meiracker AH, van Urk H, Roelandt $J R$, Poldermans D. Comparison between serum creatinine and creatinine clearance for the prediction of postoperative mortality in patients undergoing major vascular surgery. Clin Nephrol 2003; 59:17-23.

179 Welten GM, Schouten O, Chonchol M, Hoeks SE, Feringa HH, Bax JJ, Dunkelgrun M, van Gestel YR, van Domburg RT, Poldermans D. Temporary worsening of renal function after aortic surgery is associated with higher long-term mortality. Am J Kidney Dis 2007; 50:219-228.

180 Kheterpal S, Tremper KK, Englesbe MJ, O'Reilly M, Shanks AM, Fetterman DM, Rosenberg AL, Swartz RD. Predictors of postoperative acute renal failure after noncardiac surgery in patients with previously normal renal function. Anesthesiology 2007; 107:892-902.

181 McCullough PA, Soman SS. Contrast-induced nephropathy. Crit Care Clin 2005; 21:261-280.
182 Merten GJ, Burgess WP, Gray LV, Holleman JH, Roush TS, Kowalchuk GJ, Bersin RM, Van Moore A, Simonton CA 3rd, Rittase RA, Norton HJ, Kennedy TP. Prevention of contrast-induced nephropathy with sodium bicarbonate: a randomized controlled trial. JAMA 2004; 291:2328-2334.

183 Maioli M, Toso A, Leoncini M, Gallopin M, Tedeschi D, Micheletti C, Bellandi F. Sodium bicarbonate versus saline for the prevention of contrast-induced nephropathy in patients with renal dysfunction undergoing coronary angiography or intervention. J Am Coll Cardiol 2008; 52:599-604.

184 Salem DN, Stein PD, Al-Ahmad A, Bussey HI, Horstkotte D, Miller N, Pauker SG. Antithrombotic therapy in valvular heart disease-native and prosthetic: the Seventh ACCP Conference on Antithrombotic and Thrombolytic Therapy. Chest 2004; 126 (3 Suppl):457S-482S.

185 Murkin JM. Neurologic complications in noncardiac surgery. Semin Cardiothorac Vasc Anesth 2006; 10:125-127.

186 Newman S, Stygall J, Hirani S, Shaefi S, Maze M. Postoperative cognitive dysfunction after noncardiac surgery: a systematic review. Anesthesiology 2007; 106:572-590.

187 Selim M. Perioperative stroke. N Engl J Med 2007; 356:706-713.

188 Armstrong MJ, Schneck MJ, Biller J. Discontinuation of perioperative antiplatelet and anticoagulant therapy in stroke patients. Neurol Clin 2006; 24:607-630.

189 Sin DDWL, Man SF. The relationship between reduced lung function and cardiovascular mortality. A population based study and a systview of the literature. Chest 2005; 127:1952-1959.

190 Ramakrishna GSJ, Ravi BS, Chandrasekaran K, McGoon MD. Impact of pulmonary hypertension on the outcomes of non cardiac surgery: predictors of perioperative morbidity and mortality. J Am Coll Cardiol 2005; 45:1691-1699.

191 Welten GM, Schouten O, van Domburg RT, Feringa HH, Hoeks SE, Dunkelgrun M, van Gestel YR, Goei D, Bax JJ, Poldermans D. The influence of aging on the prognostic value of the revised cardiac risk index for postoperative cardiac complications in vascular surgery patients. Eur J Vasc Endovasc Surg 2007; 34:632-638.

192 van Gestel YR, Hoeks SE, Sin DD, Simsek C, Welten GM, Schouten O, Stam H, Mertens FW, van Domburg RT, Poldermans D. Effect of statin therapy on mortality in patients with peripheral arterial disease and comparison of those with versus without associated chronic obstructive pulmonary disease. Am J Cardio/ 2008; 102:192-196.

193 Galie' N, Hoeper NM, Humbert M, Torbicki A, Vachiery J-C, Barbera JA, Beghetti M, Corris P, Gaine S, Gibbs JS, Gomez-Sanchez MA, Jondeau G, Klepetko W, Opitz C, Peacock A, Rubin L, Zellweger M, Simonneau G. Guidelines for the diagnosis and treatment of pulmonary hypertension. The Task Force for the Diagnosis and Treatment of Pulmonary Hypertension of the European Society of Cardiology (ESC) and the European Respiratory Society (ERS), endorsed by the International Society of Heart and Lung Transplantation (ISHLT). Eur Heart J 2009; in press, doi:10.1093/eurheartj/ehp297.

194 Biagini A, L'Abbate A, Testa R, Carpeggiani C, Mazzei MG, Michelassi C, Benassi A, Riva A, Marchesi C, Maseri A. Unreliability of conventional visual electrocardiographic monitoring for detection of transient ST segment changes in a coronary care unit. Eur Heart $J$ 1984; 5:784-791.

195 Leung JM, O'Kelly B, Browner WS, Tubau J, Hollenberg M, Mangano DT. Prognostic importance of postbypass regional wall-motion abnormalities in patients undergoing coronary artery bypass graft surgery. SPI Research Group. Anesthesiology 1989; 71:16-25.

196 Leung JM, Voskanian A, Bellows WH, Pastor D. Automated electrocardiograph ST segment trending monitors: accuracy in detecting myocardial ischemia. Anesth Analg 1998; 87:4-10.

197 Kaplan JA, King SB 3rd. The precordial electrocardiographic lead (V5) in patients who have coronary-artery disease. Anesthesiology 1976; 45:570-574.

198 London MJ, Hollenberg M, Wong MG, Levenson L, Tubau JF, Browner W, Mangano DT. Intraoperative myocardial ischemia: localization by continuous 12-lead electrocardiography. Anesthesiology 1988; 69:232241.

199 Landesberg G, Mosseri M, Wolf Y, Vesselov Y, Weissman C. Perioperative myocardial ischemia and infarction: identification by continuous 12-lead electrocardiogram with online ST-segment monitoring. Anesthesiology 2002; 96:264-270.

200 Kertai MD, Boersma E, Klein J, Van Urk H, Bax JJ, Poldermans D. Longterm prognostic value of asymptomatic cardiac troponin T elevations in patients after major vascular surgery. Eur J Vasc Endovasc Surg 2004; 28:59-66.

201 Landesberg G, Shatz V, Akopnik I, Wolf YG, Mayer M, Berlatzky Y, Weissman C, Mosseri M. Association of cardiac troponin, CK-MB, and postoperative myocardial ischemia with long-term survival after major vascular surgery. J Am Coll Cardiol 2003; 42:1547-1554. 
202 Martinez EA, Kim L, Faraday N, Rosenfeld B, Bass EB, Perler BA, Williams GM, Dorman T, Pronovost PJ. Sensitivity of routine intensive care unit surveillance for detecting myocardial ischemia. Crit Care Med 2003; 31:2302-2308.

203 Drew BJ, Krucoff MW. Multilead ST-segment monitoring in patients with acute coronary syndromes: a consensus statement for healthcare professionals. ST-Segment Monitoring Practice Guideline International Working Group. Am J Crit Care 1999; 8:372-386; quiz 387-378.

204 Landesberg G, Luria MH, Cotev S, Eidelman LA, Anner H, Mosseri M, Schechter D, Assaf J, Erel J, Berlatzky Y. Importance of long-duration postoperative ST-segment depression in cardiac morbidity after vascular surgery. Lancet 1993; 341:715-719.

205 Fleisher LA. Real-time intraoperative monitoring of myocardial ischemia in noncardiac surgery. Anesthesiology 2000; 92:1183-1188.

206 Mangano DT, Browner WS, Hollenberg M, London MJ, Tubau JF, Tateo IM. Association of perioperative myocardial ischemia with cardiac morbidity and mortality in men undergoing noncardiac surgery. The Study of Perioperative Ischemia Research Group. N Engl J Med 1990; 323:1781-1788.

207 Mangano DT, Goldman L. Preoperative assessment of patients with known or suspected coronary disease. N Engl J Med 1995; 333:17501756.

208 London MJ, Tubau JF, Wong MG, Layug E, Hollenberg M, Krupski WC, Rapp JH, Browner WS, Mangano DT. The 'natural history' of segmental wall motion abnormalities in patients undergoing noncardiac surgery. S.P.I. Research Group. Anesthesiology 1990; 73:644-655.

209 Eisenberg MJ, London MJ, Leung JM, Browner WS, Hollenberg M, Tubau JF, Tateo IM, Schiller NB, Mangano DT. Monitoring for myocardial ischemia during noncardiac surgery. A technology assessment of transesophageal echocardiography and 12-lead electrocardiography. The Study of Perioperative Ischemia Research Group. JAMA 1992; 268:210-216.

210 Practice guidelines for perioperative transesophageal echocardiography. A report by the American Society of Anesthesiologists and the Society of Cardiovascular Anesthesiologists Task Force on Transesophageal Echocardiography. Anesthesiology 1996; 84:986-1006.

211 Polanczyk CA, Rohde LE, Goldman L, Cook EF, Thomas EJ, Marcantonio ER, Mangione CM, Lee TH. Right heart catheterization and cardiac complications in patients undergoing noncardiac surgery: an observational study. JAMA 2001; 286:309-314.

212 Sandham JD, Hull RD, Brant RF, Knox L, Pineo GF, Doig CJ, Laporta DP, Viner S, Passerini L, Devitt H, Kirby A, Jacka M. A randomized, controlled trial of the use of pulmonary-artery catheters in high-risk surgical patients. N Engl J Med 2003; 348:5-14.

213 Feringa $\mathrm{HH}$, Vidakovic R, Karagiannis SE, Dunkelgrun M, Elhendy A, Boersma E, van Sambeek MR, Noordzij PG, Bax JJ, Poldermans D. Impaired glucose regulation, elevated glycated haemoglobin and cardiac ischaemic events in vascular surgery patients. Diabet Med 2008; 25:314-319.

214 Noordzij PG, Boersma E, Schreiner F, Kertai MD, Feringa HHH, Dunkelgrun M, Bax JJ, Klein J, Poldermans D. Increased preoperative glucose levels are associated with perioperative mortality in patients undergoing noncardiac, nonvascular surgery. Eur J Endocrinol 2007; 156:137-142.

215 International Diabetes Federation. Diabetes atlas. 2006.

216 Umpierrez GE, Isaacs SD, Bazargan N, You X, Thaler LM, Kitabchi AE. Hyperglycemia: an independent marker of in-hospital mortality in patients with undiagnosed diabetes. J Clin Endocrinol Metab 2002; 87:978982.

217 Vanhorebeek I, Langouche L, Van den Berghe G. Tight blood glucose control with insulin in the ICU: facts and controversies. Chest 2007; 132:268-278.

218 van den Berghe G, Wouters P, Weekers F, Verwaest C, Bruyninckx F, Schetz M, Vlasselaers D, Ferdinande $\mathrm{P}$, Lauwers $\mathrm{P}$, Bouillon R. Intensive insulin therapy in the critically ill patients. N Engl J Med 2001; 345:13591367.
219 Van den Berghe G, Wilmer A, Hermans G, Meersseman W, Wouters PJ, Milants I, Van Wijngaerden E, Bobbaers $H$, Bouillon R. Intensive insulin therapy in the medical ICU. N Engl J Med 2006; 354:449-461.

220 Gandhi GY, Nuttall GA, Abel MD, Mullany CJ, Schaff HV, O'Brien PC, Johnson MG, Williams AR, Cutshall SM, Mundy LM, Rizza RA, McMahon $M M$. Intensive intraoperative insulin therapy versus conventional glucose management during cardiac surgery: a randomized trial. Ann Intern Med 2007; 146:233-243.

221 Ammori JB, Sigakis M, Englesbe MJ, O'Reilly M, Pelletier SJ. Effect of intraoperative hyperglycemia during liver transplantation. J Surg Res 2007; 140:227-233.

222 Ellger B, Debaveye Y, Vanhorebeek I, Langouche L, Giulietti A, Van Etten E, Herijgers P, Mathieu C, Van den Berghe G. Survival benefits of intensive insulin therapy in critical illness: impact of maintaining normoglycemia versus glycemia-independent actions of insulin. Diabetes 2006; 55:1096-1105.

223 Finfer S, Chittock DR, Su SY, Blair D, Foster D, Dhingra V, Bellomo R, Cook D, Dodek P, Henderson WR, Hebert PC, Heritier S, Heyland DK, McArthur C, McDonald E, Mitchell I, Myburgh JA, Norton R, Potter J, Robinson BG, Ronco JJ. Intensive versus conventional glucose control in critically ill patients. N Engl J Med 2009; 360:1283-1297.

224 De Hert SG, Turani F, Mathur S, Stowe DF. Cardioprotection with volatile anesthetics: mechanisms and clinical implications. Anesth Analg 2005; 100:1584-1593.

225 Slogoff S, Keats AS. Randomized trial of primary anesthetic agents on outcome of coronary artery bypass operations. Anesthesiology 1989; 70:179-188.

226 Rodgers A, Walker N, Schug S, McKee A, Kehlet H, van Zundert A, Sage D, Futter M, Saville G, Clark T, MacMahon S. Reduction of postoperative mortality and morbidity with epidural or spinal anaesthesia: results from overview of randomised trials. BMJ 2000; 321:1493.

227 Wu CL, Rowlingson AJ, Herbert R, Richman JM, Andrews RA, Fleisher LA. Correlation of postoperative epidural analgesia on morbidity and mortality after colectomy in Medicare patients. J Clin Anesth 2006; 18:594-599.

228 Bode RH Jr, Lewis KP, Zarich SW, Pierce ET, Roberts M, Kowalchuk GJ, Satwicz PR, Gibbons GW, Hunter JA, Espanola CC. Cardiac outcome after peripheral vascular surgery. Comparison of general and regional anesthesia. Anesthesiology 1996; 84:3-13.

229 Mauermann WJ, Shilling AM, Zuo Z. A comparison of neuraxial block versus general anesthesia for elective total hip replacement: a metaanalysis. Anesth Analg 2006; 103:1018-1025.

230 Rigg JR, Jamrozik K, Myles PS, Silbert BS, Peyton PJ, Parsons RW, Collins KS. Epidural anaesthesia and analgesia and outcome of major surgery: a randomised trial. Lancet 2002; 359:1276-1282.

231 Birkmeyer JD, Stukel TA, Siewers AE, Goodney PP, Wennberg DE, Lucas FL. Surgeon volume and operative mortality in the United States. N Eng/ J Med 2003; 349:2117-2127.

232 Liu SS, Wu CL. The effect of analgesic technique on postoperative patient-reported outcomes including analgesia: a systematic review. Anesth Analg 2007; 105:789-808.

233 White PF, Kehlet $\mathrm{H}$. Postoperative pain management and patient outcome: time to return to work!. Anesth Analg 2007; 104:487-489.

234 Polomano RC, Rathmell JP, Krenzischek DA, Dunwoody CJ. Emerging trends and new approaches to acute pain management. $J$ Perianesth Nurs 2008; 23 (1 Suppl):S43-S53.

235 Dolin SJ, Cashman JN, Bland JM. Effectiveness of acute postoperative pain management: I. Evidence from published data. Br J Anaesth 2002; 89:409-423.

236 Jorgensen H, Wetterslev J, Moiniche S, Dahl JB. Epidural local anaesthetics versus opioid-based analgesic regimens on postoperative gastrointestinal paralysis, PONV and pain after abdominal surgery. Cochrane Database Syst Rev 2000; (4):CD001893.

237 Block BM, Liu SS, Rowlingson AJ, Cowan AR, Cowan JA Jr, Wu CL. Efficacy of postoperative epidural analgesia: a meta-analysis. JAMA 2003; 290:2455-2463.

238 Schug SA, Manopas A. Update on the role of non-opioids for postoperative pain treatment. Best Pract Res Clin Anaesthesiol 2007; 21:15-30. 